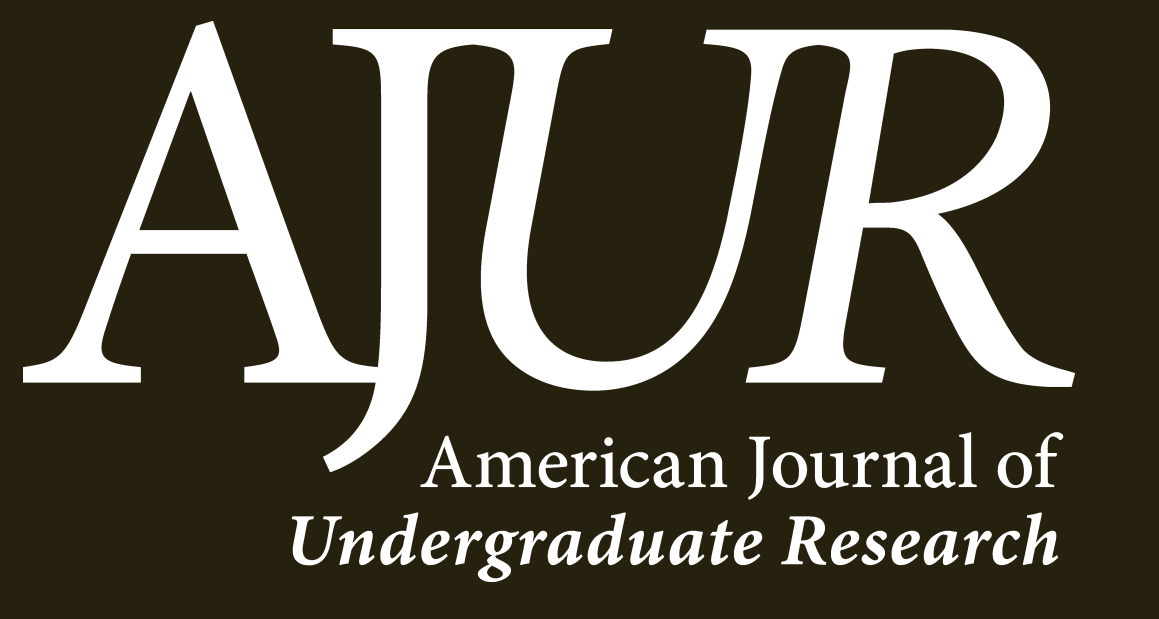

Volume 12 | Issue 4 | November 2015 www.ajuronline.org 


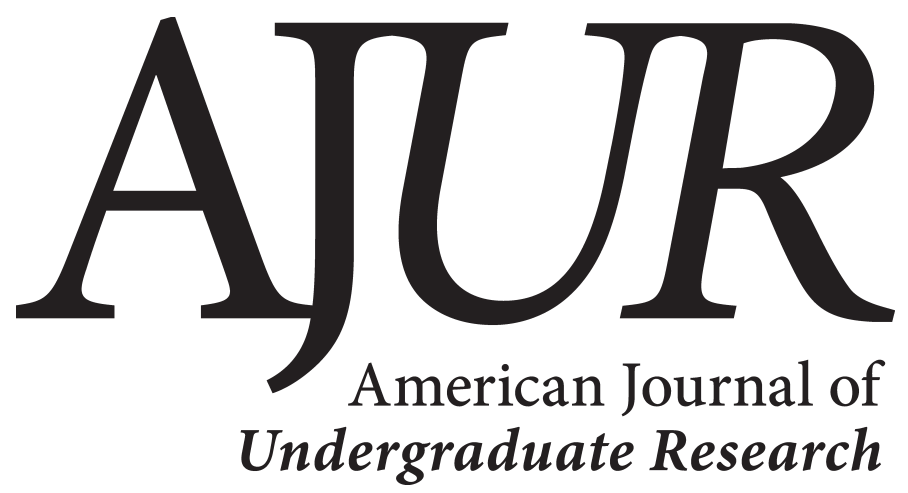

Volume 12 | Issue 4 | November 2015

www.ajuronline.org

2
Guest Editorial: A Historical Context for Undergraduate Research: The Contribution of Wilhelm von Humboldt David Vampola Special Thanks to AJUR's Sponsors

AJUR History and Editorial Board

Category Theoretic Interpretation of Rings

Edward Poon

Effect of Manual Ivy Removal on Seedling Recruitment in Forest Park, Portland, OR

Katelin D. Stanley \& Dr. David W. Taylor

Imaging Noisy Seismic Data using a One Dimensional Inverse

Scattering Algorithm

Bogdan G. Nita \& Christopher Smith

Effect of Native American Bean-corn Biculture Planting on

Free-living Bacterial Abundance and Plant Growth

Heather A. Miller, Justin Fiene \& Tamara L. Marsh

Point-Spread Function (PSF) Photometric Analysis of

Open Clusters: Melotte 72 \& NGC 2158

Muhammad Awais Mirza \& Zain Rahim

Synthesis of a MUC1 Mucin Cyclic Dimer Peptide and Its Antibody

Binding Properties as Revealed by STD-NMR

Cheng Her \& Thao Yang

Methods for Essential Tremor Assessment: Acoustic Tremor

Monitoring (ATM) and Rhythmic Spirals (RS) Methods

Emily Hart, Caroline Chow, Patricia Stan \& Daniel King 


\section{G UEST E D I T O R I A L \\ A Historical Context for Undergraduate Research: The Contribution of Wilhelm von Humboldt}

Contemporary America has sometimes been characterized as having at its heart a kind of "culture of amnesia", one in which historical precedents are often ignored in favor of the demands of the present. This narrow viewpoint can be extended to the images of "research" that people often have in this country these are of men and women performing experiments while wearing white coats and goggles in a shiny laboratory with elaborate instrumentation of various kinds. Yet, research is more than just an activity that takes place under certain prescribed conditions - it is also a type of cognitive and educational process that can express the ideals of learning. For persons interested in understanding this larger context of research in the environment of higher education, the perspective of the late eighteenth and early nineteenth century "Renaissance Man", Wilhelm von Humboldt, is of paramount importance.

Wilhelm von Humboldt (who should not be confused with his naturalist/scientist brother, Alexander) contributed to several fields of knowledge, but it was in his role as head of the section for ecclesiastic affairs and education in the Prussian ministry of the interior in the early nineteenth century that he had perhaps the largest practical impact. As difficult as it is to imagine now, many European universities of that time period were in a state of relative decline in terms of conveying important intellectual developments to the students who were enrolled in them. Von Humboldt believed that although teaching was of central importance to the mission of the university, it could only be truly effective if the activities and results of research informed what was conveyed to students. Hence, there are two entwined intellectual goals for universities: teaching and research.

Simply asserting the importance of research in the activities of an effective university was not enough for von Humboldt. The cognitive effect of this amalgam of research and education upon the individual student is also important. The effect that is identified with the end, or purpose, of university education in expressed in what von Humboldt called "Bildung". The meaning of this word, as has been often noted, is not easily rendered into English, but it is often translated as "self cultivation". At a surface level, without making further inquiry into the deeper cultural significance of this term, "self cultivation" can refer to the autonomous development of an individual's mind.

Now, how can the thoughts of von Humboldt that were meant to articulate the ideals for early nineteenth century education help with appreciating the importance of research - particularly for undergraduatesin the twenty-first century context? On an obvious level, von Humboldt saw the important singular role that research can play within the workings of institutions of higher learning. But, more importantly, the combination of research with teaching was to have a fundamental influence on the minds of students. Through engagement with research, either indirectly by listening to a professor's lectures or directly by engaging with the work of creation, interpretation, experimentation and analysis, the student's mind would develop its independent and critical capacity. Without research, this essential developmental aspect of a person's education could not be completed. But beyond the individual student's intellectual growth, the process of cultivation involves the role of faculty, since "cultivation" can involve "enculturation" (through the agency of instructors who combine teaching and research) into the collective enterprise of learning.

Our society needs, for so many different reasons, to have students develop independent, critical and creative minds. Two centuries ago Wilhelm von Humboldt saw the importance of learning about the latest intellectual trends - and also the act of doing research - as central to this educational process. Today, undergraduates need to continue the legacy of von Humboldt in order to not only fully develop their own minds, but also to achieve their full potential as persons in the human community.

David Vampola teaches in the Department of Computer Science, as well as in the Cognitive Science, Information Science, Integrated Media and Human-Computer Interaction programs at SUNY Oswego. He has given presentations (partial list) at Boston University, Ecole Normale Superieure, Centre National de la Recherche Scientifique, University of Osnabrück, Leo Baack Institute, New York Academy of Sciences, and the American Association for Higher Education, as well as at a number of international conferences. His publications have ranged over topics from the conceptual foundations of science to the statistical analysis of the health professions. He has held teaching or research positions at Boston University, Brown University Medical School, and the University of Pittsburgh. 


\section{SPECIAL THA N KS}

AJUR is made possible through the assistance of our sponsors.

Support for this issue has been provided by the Office of the Provost, at the State University of New York at Oswego.

Thank you!

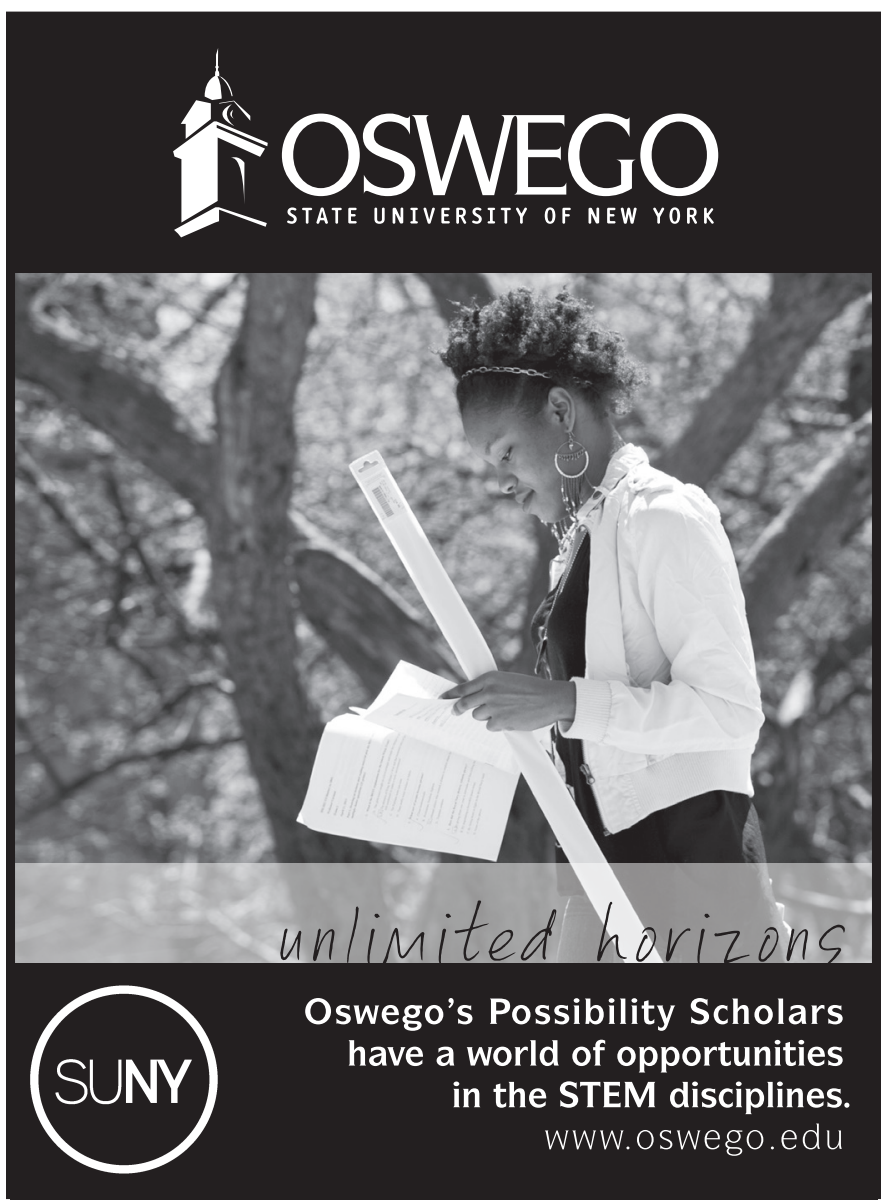

Interested in supporting quality undergraduate research? Request sponsorship information at editor@ajuronline.org 
American Journal of Undergraduate Research (AJUR) is a national, peer-reviewed, open-source, quarterly, multidisciplinary student research journal. It is indexed internationally by EBSCO, and is listed via the Library of Congress under ISSNs of 1536-4585 (for print) and 2375-8732 (for web). The journal was established in 2002.

EDITORIAL TEAM Volume 12 | Issue 4 | November 2015

Dr. Kestutis G. Bendinskas, Editor, editor@ajuronline.org

Dr. Anthony Contento, Assistant Technical Editor, technicaleditor@ajuronline.org

Rose Throop, Art and Copy Editor, copyeditor@ajuronline.org

Daniel Laird, Web Master,webmaster@ajuronline.org

EDITORIAL BOARD by subject area

\begin{tabular}{|c|}
\hline ACCOUNTING \\
\hline $\begin{array}{l}\text { Dr. Dean Crawford, } \\
\text { dean.crawford@oswego.edu }\end{array}$ \\
\hline ART HISTORY \\
\hline $\begin{array}{l}\text { Dr. Lisa Seppi, } \\
\text { lisa.seppi@oswego.edu }\end{array}$ \\
\hline ASTROPHYSICS \\
\hline $\begin{array}{l}\text { Dr. Shashi Kanbur, } \\
\text { shashi.kanbur@oswego.edu }\end{array}$ \\
\hline BEHAVIORAL NEUROSCIENCE \\
\hline $\begin{array}{l}\text { Dr. Aileen M. Bailey, } \\
\text { ambailey@smcm.edu }\end{array}$ \\
\hline BIOCHEMISTRY \\
\hline $\begin{array}{l}\text { Dr. Pamela K. Kerrigan, } \\
\text { pamela.kerrigan@mountsaintvincent.edu }\end{array}$ \\
\hline BIOENGINEERING \\
\hline $\begin{array}{l}\text { Dr. Jorge I. Rodriguez, } \\
\text { jorger@clemson.edu }\end{array}$ \\
\hline BIOINFORMATICS \\
\hline $\begin{array}{l}\text { Dr. Kevin Daimi, } \\
\text { daimikj@udmercy.edu }\end{array}$ \\
\hline $\begin{array}{l}\text { Dr. John R. Jungck, } \\
\text { jungck@udel.edu }\end{array}$ \\
\hline BIOLOGY, PHYSIOLOGY \\
\hline $\begin{array}{l}\text { Dr. David Dunn, } \\
\text { david.dunn@oswego.ed }\end{array}$ \\
\hline BIOLOGY, DEVELOPMENTAL \\
\hline $\begin{array}{l}\text { Dr. Poongodi Geetha-Loganathan, } \\
\text { p.geethaloganathan@oswego.edu }\end{array}$ \\
\hline BIOLOGY, MICROBIOLOGY \\
\hline $\begin{array}{l}\text { Dr. Peter Newell, } \\
\text { peter.newell@oswego.edu }\end{array}$ \\
\hline BOTANY \\
\hline $\begin{array}{l}\text { Dr.William R. Bromer, } \\
\text { wbromer@stfrancis.edu }\end{array}$ \\
\hline $\begin{array}{l}\text { Dr. Julien Bachelier, } \\
\text { julien.bachelier@oswego.edu }\end{array}$ \\
\hline
\end{tabular}

\section{CHEMISTRY}

Dr Alfredo Castro, castroa@felician.edu

Dr. Charles Kriley,

cekriley@gcc.edu

Dr. Douglas Mulford,

douglas.mulford@emory.edu

Dr. Mark A. Benvenuto,

benvenma@udmercy.edu

\section{CHEMISTRY, PHYSICAL}

Dr. John Oakes,

john.oakes@gcccd.net

\section{COMPUTATIONAL SCIENCES}

Dr. Dele Oluwade,

deleoluwade@yahoo.com

Dr. J. Ben Schafer,

schafer@cs.uni.edu

\section{ECOLOGY}

Dr. William R. Bromer,

wbromer@stfrancis.edu

\section{EDUCATION, PHYSICS}

Dr. Andrew D. Gavrin,

agavrin@iupui.edu

\section{ENGINEERING, ENVIRONMENTAL}

Dr. Eileen M. Cashman,

eileen.cashman@humboldt.edu

\section{ENVIRONMENTAL SCIENCE}

Dr. Eileen M. Cashman,

eileen.cashman@humboldt.edu

\section{GEOLOGY}

Dr. Larry Davis,

ldavis@csbsju.edu

\section{HONORARY EDITORIAL}

\section{BOARD MEMBER}

Dr. Lorrie Clemo,

lorrie.clemo@oswego.edu

\section{KINESIOLOGY / EXERCISE SCIENCE}

Dr. David Senchina,

david.senchina@drake.edu

LITERARY STUDIES

Dr. Douglas Guerra,

douglas.guerra@oswego.edu
MATHEMATICS

Dr. Nkechi M. Agwu, nagwu@bmcc.cuny.edu

Dr. John Emert,

emert@bsu.edu

Dr. Jeffrey J. Boats,

boatsjj@udmercy.edu

Dr. J.D. Phillips,

jophilli@nmu.edu

Dr. Dele Oluwade,

deleoluwade@yahoo.com

METEOROLOGY

Dr. Steven Skubis,

steven.skubis@oswego.edu

\section{NEUROSCIENCE}

Dr. Pamela E. Scott-Johnson, pamela.scottjohnson@morgan.edu

Dr. Amy Overman,

aoverman@elon.edu

PHYSICS

Dr. Carolina Ilie, carolina.ilie@oswego.edu

\section{PSYCHOLOGY}

Dr. Amy Overman, aoverman@elon.edu

Dr. Pamela E. Scott-Johnson,

pamela.scottjohnson@morgan.edu

\section{STATISTICS}

Dr. Mark Ecker,

mark.ecker@uni.edu

\section{TECHNOLOGY, ENGINEERING}

Dr. Recayi Pecen,

regpecen@na.edu 


\title{
Category Theoretic Interpretation of Rings
}

\author{
Edward Poon \\ Department of Mathematics and Statistics, University of Ottawa, ON, Canada \\ Student:epoon061@uottawa.ca, Mentor: Alistair Savage
}

\begin{abstract}
We enhance the category of rings and the category of idempotented rings to 2-categories. After doing this, we prove an equivalence of 1-categories and 2-categories between the category of rings and the category of small preadditive categories with one object and between the category of idempotented rings and the category of small preadditive categories with finitely many objects. Under these equivalences, we demonstrate some analogues between notions in category theory and ring theory.
\end{abstract}

\section{KEYWORDS}

Ring, Idempotent, Preadditive Category

\section{INTRODUCTION}

One of the most famous problems in mathematics was the proof for Fermat's Last Theorem, dating back to 1637, that states that there do not exist three positive integers $a, b, c$ such that $a^{n}+b^{n}=c^{n}$ for an integer $n$ greater than two. ${ }^{1}$ Through attempts to prove this theorem, the concept of a ring was introduced by Richard Dedekind in the 1800's which provided a generalization of arithmetic. However, it was not until the 1920's that (commutative) rings were axiomatically defined by Emmy Noether and Wolfgang Krull in their theory of ideals. ${ }^{2}$ Ring theory has since grown to be an active field of research with interesting connections to algebraic number theory and algebraic geometry.

In comparison to a ring, the concept of a category is much younger with category theory being a field of mathematics introduced by Samuel Eilenberg and Saunders Mac Lane in 1945 as part of their work in topology. ${ }^{3}$ However, applications to other fields of mathematics have since grown tremendously. Notably, Alexander Grothendieck almost single-handedly shaped modern algebraic geometry with the use of category theory whereas William Lawvere applied category theory to logic to develop the field of categorical logic. ${ }^{4,5}$ While there are many diverse uses of category theory, applications to abstract algebra are especially interesting since one can interpret various algebraic structures such as sets, monoids and groups as categories and vice-versa in an effort to study them in an uniform fashion. By doing so, one can translate propositions proven in categories into results in their respective algebraic structures.

In the literature, especially in the field of categorification, one often views a ring together with a collection of idempotents as a category with an object for each idempotent. For instance, this is the point of view taken in the recent categorification of quantum groups, where one typically categorifies the modified enveloping algebra of a quantum group. ${ }^{6-11}$ This algebra has an idempotent for every element of the weight lattice. We adopt this approach in the current paper. Our goal is to make the connection between rings (with idempotents) and categories as precise as possible, and create a dictionary between the two points of view. In particular, we show how we can view a ring as a small preadditive category with one object and an idempotented ring as a small preadditive category with finitely many objects. We then prove an equivalence of 1-categories and 2-categories between the category of rings and the category of small preadditive categories with one object and between the category of idempotented rings and the category of small preadditive categories with 
finitely many objects. Under these equivalences, we show in Proposition 6.3 that two functors between two small preadditive categories with one object form an adjunction if and only if there exist specific 2-morphisms of rings and mutually inverse bijections of sets.

\section{BACKGROUND ON RING THEORY}

In this section, we recall the definitions of a ring as well as different types of idempotents and systems of such idempotents within a ring. Furthermore, the notion of an idempotented ring is introduced and the example of a matrix ring is given. Lastly, we give a proof that every idempotented ring is isomorphic to a matrix ring.

Definition 2.1 (Ring)

A ring is a set $R$ equipped with two binary operations (denoted by addition and multiplication) satisfying the following axioms:

- (Commutativity of addition) For all $a, b \in R, a+b=b+a$.

- (Associativity of addition) For all $a, b, c \in R, a+(b+c)=(a+b)+c$.

- (Additive identity) There exists an additive identity $0_{R} \in R$ such that for all $a \in R, 0_{R}+a=a=a+0_{R}$.

- (Additive inverse) For all $a \in R$, there exists an element $-a \in R$ such that $a+(-a)=0_{R}$.

- (Associativity of multiplication) For all $a, b, c \in R, a \cdot(b \cdot c)=(a \cdot b) \cdot c$.

- (Multiplicative identity) There exists a multiplicative identity $1_{R} \in R$ such that for all $a \in R, 1_{R} \cdot a=a=a \cdot 1_{R}$.

- (Left distributive property) For all $a, b, c \in R, a \cdot(b+c)=a \cdot b+a \cdot c$.

- (Right distributive property) For all $a, b, c \in R,(b+c) \cdot a=b \cdot a+c \cdot a$.

Definition 2.2 (Idempotent, orthogonal idempotent)

An element $e$ in a ring $R$ is idempotent if $e^{2}=e$. Idempotents $e$ and $e^{\prime}$ in $R$ are a pair of orthogonal idempotents if $e \cdot e^{\prime}=e^{\prime} \cdot e=0$.

Definition 2.3 (Complete multiset of orthogonal idempotents)

Let $R$ be a ring with a multiset $I=\left\{e_{1}, e_{2}, \cdots, e_{n}\right\} \in R$. We call $I$ a complete multiset of orthogonal idempotents if:

- For distinct $e_{i}, e_{j}$, in $I, e_{i}, e_{j}$, is a pair of orthogonal idempotents.

- $1_{R}=e_{1}+e_{2}+\cdots+e_{n}$.

Definition 2.4 (Idempotented ring)

An idempotented ring is a pair $(R, I)$, where $R$ is a ring and $I$ a complete multiset of orthogonal idempotents. 
Example 2.5 (Matrix ring)

Suppose $(R, I)$ is an idempotented ring with $I=\left\{e_{1}, e_{2}, \cdots, e_{n}\right\}$. We define a ring $M_{n}(R)$ with multiplicative and additive identities $1_{M_{n}(R)}$ and $0_{M_{n}(R)}$ as follows.

$$
\begin{aligned}
& M_{n}(R)=\left\{\left(\begin{array}{cccc}
e_{1} r e_{1} & e_{1} r e_{2} & \cdots & e_{1} r e_{n} \\
e_{2} r e_{1} & e_{2} r e_{2} & \cdots & e_{2} r e_{n} \\
\vdots & \vdots & \ddots & \vdots \\
e_{n} r e_{1} & e_{n} r e_{2} & \cdots & e_{n} r e_{n}
\end{array}\right): r \in R\right\}, \\
& 1_{M_{n}(R)}=\left(\begin{array}{cccc}
e_{1} & 0_{R} & \cdots & 0_{R} \\
0_{R} & e_{2} & \cdots & 0_{R} \\
\vdots & \vdots & \ddots & \vdots \\
0_{R} & 0_{R} & \cdots & e_{n}
\end{array}\right), 0_{M_{n}(R)}=\left(\begin{array}{cccc}
0_{R} & 0_{R} & \cdots & 0_{R} \\
0_{R} & 0_{R} & \cdots & 0_{R} \\
\vdots & \vdots & \ddots & \vdots \\
0_{R} & 0_{R} & \cdots & 0_{R}
\end{array}\right) .
\end{aligned}
$$

Next, we define a multiset $I_{M_{n}(R)} \subseteq M_{n}(R)$ in this fashion,

$$
I_{M_{n}(R)}=\left\{\left(\begin{array}{cccc}
e_{1} & 0_{R} & \cdots & 0_{R} \\
0_{R} & 0_{R} & \cdots & 0_{R} \\
\vdots & \vdots & \ddots & \vdots \\
0_{R} & 0_{R} & \cdots & 0_{R}
\end{array}\right),\left(\begin{array}{cccc}
0_{R} & 0_{R} & \cdots & 0_{R} \\
0_{R} & e_{2} & \cdots & 0_{R} \\
\vdots & \vdots & \ddots & \vdots \\
0_{R} & 0_{R} & \cdots & 0_{R}
\end{array}\right), \cdots,\left(\begin{array}{cccc}
0_{R} & 0_{R} & \cdots & 0_{R} \\
0_{R} & 0_{R} & \cdots & 0_{R} \\
\vdots & \vdots & \ddots & \vdots \\
0_{R} & 0_{R} & \cdots & e_{n}
\end{array}\right)\right\} .
$$

It is fairly simple to prove that $M_{n}(R)$ is a ring since most of the ring axioms follow from the properties of matrices and so we will omit the proof. Likewise, it is also straightforward to prove that every pair of $M, N \in I_{M_{n}(R)}$ is pairwise orthogonal and that the sum of all $M \in I_{M_{n}(R)}=1_{M_{n}(R) \text {. }}$ Thus, $I_{M_{n}(R)}$ is a complete multiset of orthogonal idempotents.

Proposition 2.6 (Every idempotented ring is isomorphic to a matrix ring)

Proof.

Let $(R, I)$ be an idempotented ring and $\left(M_{n}(R), I_{M_{n}}(I)\right)$ be the matrix ring over $(R, I)$. We define $\phi:(R, I) \rightarrow\left(M_{n}(R), I_{M_{n}(R)}\right)$ to be the map $r \mapsto\left(e_{j} r e_{i}\right)$ where $\left(e_{j} r e_{i}\right)$ denotes the $n \times n$ matrix whose $(i, j)$ entry is $e_{j} e_{i}$. We claim that $\phi$ is a ring isomorphism.

- (Injective) Assume that for some $r$ and $r^{\prime}$ in $R, \phi(r)=\phi\left(r^{\prime}\right)$. Then for any index $(i, j)$, $r=\left(e_{1}+\cdots+e_{n}\right) r\left(e_{1}+\cdots+e_{n}\right)=\sum_{i, j=1}^{n} e_{i} r e_{j}=\sum_{i, j=1}^{n} e_{i} r^{\prime} e_{j}$

$$
=\left(e_{1}+\cdots+e_{n}\right) r^{\prime}\left(e_{1}+\cdots+e_{n}\right)=r^{\prime} .
$$

- (Surjective) By the definition of $M_{n}(R)$, for any matrix $M \in M_{n}(R), \phi(r)=M$ for some $r \in R$.

- (Preserves Sums) Let $r, r^{\prime} \in R$, then we have the following set of equalities.

$$
\begin{aligned}
\phi\left(r+r^{\prime}\right) & =\left(\begin{array}{cccc}
e_{1}\left(r+r^{\prime}\right) e_{1} & e_{1}\left(r+r^{\prime}\right) e_{2} & \cdots & e_{1}\left(r+r^{\prime}\right) e_{n} \\
e_{2}\left(r+r^{\prime}\right) e_{1} & e_{2}\left(r+r^{\prime}\right) e_{2} & \cdots & e_{2}\left(r+r^{\prime}\right) e_{n} \\
\vdots & \vdots & \ddots & \vdots \\
e_{n}\left(r+r^{\prime}\right) e_{1} & e_{n}\left(r+r^{\prime}\right) e_{2} & \cdots & e_{n}\left(r+r^{\prime}\right) e_{n}
\end{array}\right) \\
& =\left(\begin{array}{cccc}
e_{1} r e_{1} & e_{1} r e_{2} & \cdots & e_{1} r e_{n} \\
e_{2} r e_{1} & e_{2} r e_{2} & \cdots & e_{2} r e_{n} \\
\vdots & \vdots & \ddots & \vdots \\
e_{n} r e_{1} & e_{n} r e_{2} & \cdots & e_{n} r e_{n}
\end{array}\right)+\left(\begin{array}{cccc}
e_{1} r^{\prime} e_{1} & e_{1} r^{\prime} e_{2} & \cdots & e_{1} r^{\prime} e_{n} \\
e_{2} r^{\prime} e_{1} & e_{2} r^{\prime} e_{2} & \cdots & e_{2} r^{\prime} e_{n} \\
\vdots & \vdots & \ddots & \vdots \\
e_{n} r^{\prime} e_{1} & e_{n} r^{\prime} e_{2} & \cdots & e_{n} r^{\prime} e_{n}
\end{array}\right) \\
& =\phi(r)+f\left(r^{\prime}\right) .
\end{aligned}
$$


- (Preserves Products) Let $r, r^{\prime} \in R$, then we have the following set of equalities.

$$
\begin{aligned}
\phi(r) \cdot \phi\left(r^{\prime}\right) & =\left(\begin{array}{cccc}
e_{1} r e_{1} & e_{1} r e_{2} & \cdots & e_{1} r e_{n} \\
e_{2} r e_{1} & e_{2} r e_{2} & \cdots & e_{2} r e_{n} \\
\vdots & \vdots & \ddots & \vdots \\
e_{n} r e_{1} & e_{n} r e_{2} & \cdots & e_{n} r e_{n}
\end{array}\right)\left(\begin{array}{cccc}
e_{1} r^{\prime} e_{1} & e_{1} r^{\prime} e_{2} & \cdots & e_{1} r^{\prime} e_{n} \\
e_{2} r^{\prime} e_{1} & e_{2} r^{\prime} e_{2} & \cdots & e_{2} r^{\prime} e_{n} \\
\vdots & \vdots & \ddots & \vdots \\
e_{n} r^{\prime} e_{1} & e_{n} r^{\prime} e_{2} & \cdots & e_{n} r^{\prime} e_{n}
\end{array}\right) \\
& =\left(\begin{array}{ccccc}
\sum_{i=1}^{n}\left(e_{1} r e_{i}\right)\left(e_{i} r^{\prime} e_{1}\right) & \sum_{i=1}^{n}\left(e_{1} r e_{i}\right)\left(e_{i} r^{\prime} e_{2}\right) & \cdots & \sum_{i=1}^{n}\left(e_{1} r e_{i}\right)\left(e_{i} r^{\prime} e_{n}\right) \\
\sum_{i=1}^{n}\left(e_{2} r e_{i}\right)\left(e_{i} r^{\prime} e_{1}\right) & \sum_{i=1}^{n}\left(e_{2} r e_{i}\right)\left(e_{i} r^{\prime} e_{2}\right) & \cdots & \sum_{i=1}^{n}\left(e_{2} r e_{i}\right)\left(e_{i} r^{\prime} e_{n}\right) \\
\vdots & \vdots & \ddots & \ddots & \vdots \\
\sum_{i=1}^{n}\left(e_{n} r e_{i}\right)\left(e_{i} r^{\prime} e_{1}\right) & \sum_{i=1}^{n}\left(e_{n} r e_{i}\right)\left(e_{i} r^{\prime} e_{2}\right) & \cdots & \sum_{i=1}^{n}\left(e_{n} r e_{i}\right)\left(e_{i} r^{\prime} e_{n}\right)
\end{array}\right) \\
& =\left(\begin{array}{ccccc}
\sum_{i=1}^{n}\left(e_{1} r e_{i} r^{\prime} e_{1}\right) & \sum_{i=1}^{n}\left(e_{1} r e_{i} r^{\prime} e_{2}\right) & \cdots & \sum_{i=1}^{n}\left(e_{1} r e_{i} r^{\prime} e_{n}\right) \\
\sum_{i=1}^{n}\left(e_{2} r e_{i} r^{\prime} e_{1}\right) & \sum_{i=1}^{n}\left(e_{2} r e_{i} r^{\prime} e_{2}\right) & \cdots & \sum_{i=1}^{n}\left(e_{2} r e_{i} r^{\prime} e_{n}\right) \\
\vdots & \vdots & \ddots & \vdots \\
\sum_{i=1}^{n}\left(e_{n} r e_{i} r^{\prime} e_{1}\right) & \sum_{i=1}^{n}\left(e_{n} r e_{i} r^{\prime} e_{2}\right) & \cdots & \sum_{i=1}^{n}\left(e_{n} r e_{i} r^{\prime} e_{n}\right)
\end{array}\right) \\
& =\left(\begin{array}{cccc}
e_{1}\left(r \cdot r^{\prime}\right) e_{1} & e_{1}\left(r \cdot r^{\prime}\right) e_{2} & \cdots & e_{1}\left(r \cdot r^{\prime}\right) e_{n} \\
e_{2}\left(r \cdot r^{\prime}\right) e_{1} & e_{2}\left(r \cdot r^{\prime}\right) e_{2} & \cdots & e_{2}\left(r \cdot r^{\prime}\right) e_{n} \\
\vdots & \vdots & \ddots & \vdots \\
e_{n}\left(r \cdot r^{\prime}\right) e_{1} & e_{n}\left(r \cdot r^{\prime}\right) e_{2} & \cdots & e_{n}\left(r \cdot r^{\prime}\right) e_{n}
\end{array}\right) \\
& \phi\left(r \cdot r^{\prime}\right)
\end{aligned}
$$

- (Preserves Identity)

$$
\phi\left(1_{R}\right)=\left(\begin{array}{ccccc}
e_{1} \cdot 1_{R} \cdot e_{1} & e_{1} \cdot 1_{R} \cdot e_{2} & \cdots & e_{1} \cdot 1_{R} \cdot e_{n} \\
e_{2} \cdot 1_{R} \cdot e_{1} & e_{2} \cdot 1_{R} \cdot e_{2} & \cdots & e_{2} \cdot 1_{R} \cdot e_{n} \\
\vdots & \vdots & \ddots & \vdots \\
e_{n} \cdot 1_{R} \cdot e_{1} & e_{n} \cdot 1_{R} \cdot e_{2} & \cdots & e_{n} \cdot 1_{R} \cdot e_{n}
\end{array}\right)=\left(\begin{array}{cccc}
e_{1} & 0_{R} & \cdots & 0_{R} \\
0_{R} & e_{2} & \cdots & 0_{R} \\
\vdots & \vdots & \ddots & \vdots \\
0_{R} & 0_{R} & \cdots & e_{n}
\end{array}\right) .
$$




\section{BACKGROUND ON CATEGORY THEORY}

In this section, we recall the notion of a category and give some examples involving algebraic structures. We then consider mappings between categories with the definition of a functor and consider relations between functors through natural transformations and adjunctions. Lastly, we give the definition of an equivalence of categories which we will use to great effect in later sections.

Definition 3.1 (Category, small category)

A category $C$ consists of a class of objects $\mathrm{ObC}$ and for every pair of objects $X, Y$, a class of morphisms $\operatorname{Mor} C(X, Y)$, writing $f: X \rightarrow Y$ to denote a morphism in $\operatorname{Mor}_{C}(X, Y)$. These classes must satisfy the following:

- (Composition) For any $X, Y, Z \in \mathrm{Ob} C$, there is a map of composition $\operatorname{Mor}_{C}(Y, Z) \times \operatorname{Mor} C(X, Y) \rightarrow \operatorname{Mor}_{C}(X, Z)$.

- (Identity) For any object $X \in \mathrm{Ob} C$, there exists an identity morphism of $X, \operatorname{id}_{X}: X \rightarrow X$, such that for any morphism $f: X \rightarrow Y, f \circ \operatorname{id}_{X}=f=\operatorname{id}_{Y} \circ f \in \operatorname{Mor}_{C}(X, Y)$.

- (Associativity) Given $f \in \operatorname{Mor} C\left(X, X^{\prime}\right), g \in \operatorname{Mor}_{C}\left(X^{\prime}, Y\right)$ and $h \in \operatorname{Mor} C\left(Y, Y^{\prime}\right)$, $(h \circ g) \circ f=h \circ(g \circ f) \in \operatorname{Mor} C\left(X, Y^{\prime}\right)$.

A category $C$ is small if the class of objects and the class of morphisms are both sets.

Example 3.2 (Category of finite-dimensional vector spaces)

Let FinVect $\mathbb{F}$ denote the category of finite-dimensional vector spaces over a fixed field $\mathbb{F}$. The objects of FinVect $\mathbb{F}$ are finite-dimensional vector spaces over $\mathbb{F}$ and for any $V, W \in$ Ob FinVect $_{\mathbb{F}}$, $\operatorname{Mor}_{\text {FinVectF }}(V, W)$ is the class of linear maps from $V$ to $W$. The axioms of a category are satisfied with the following data.

- (Identity) For any $V \in \mathrm{Ob}$ FinVect $\mathbb{F}$, id $V$ is the identity map from $V$ onto itself.

- (Composition) The composition of morphisms in FinVect $F$ is the usual composition of linear maps.

- (Associativity) Associativity holds because the composition of linear maps is associative.

Example 3.3 (Category of matrices)

Let Mat $(\mathbb{F})$ denote the category of matrices over a fixed field $\mathbb{F}$. The objects of Mat $(\mathbb{F})$ are the natural numbers, and for any $m, n \in \mathrm{Ob} \operatorname{Mat}(\mathbb{F})$, $\operatorname{Mor} \operatorname{Mat} \mathbb{F}(m, n)$ is the class of $n \times m$ matrices. The axioms of a category are satisfied with the data below.

- (Identity) For any $m \in \mathrm{ObMat}(\mathbb{F}), \mathrm{id}_{m}$ is the identity $m \times m$ matrix.

- (Composition) The composition of morphisms in $\operatorname{Mat}(\mathbb{F})$ is the multiplication of matrices.

- (Associativity) Associativity holds because the multiplication of matrices is associative.

Definition 3.4 (Isomorphism)

Suppose $C$ is a category. A morphism $f \in \operatorname{Mor}_{C}(X, Y)$ is an isomorphism between $X$ and $Y$ if there exists a morphism $g: Y \rightarrow X$ such that $f \circ g=\operatorname{id}_{Y}$ and $g \circ f=\operatorname{id}_{X}$. We say that the objects $X$ and $Y$ are isomorphic, which is denoted as $X \cong Y$. 
Definition 3.5 (Preadditive category)

A category $C$ is preadditive if for any $X, Y \in \operatorname{Ob} C, \operatorname{Mor} C(X, Y)$ has the structure of an abelian group, which we write additively. Furthermore, we require that composition is distributive over this addition. So for any $f, f^{\prime} \in \operatorname{Mor}_{C}(X, Y)$ and $g, g^{\prime} \in \operatorname{Mor}_{C}(Y, Z)$,

- $\left(g+g^{\prime}\right) \circ f=g \circ f+g^{\prime} \circ f \in \operatorname{Mor}_{C}(X, Z)$,

- $g \circ\left(f+f^{\prime}\right)=g \circ f+g \circ f^{\prime} \in \operatorname{Mor} C(X, Z)$.

Remark 3.6 We will refer to the identity with respect to the addition operation as the zero morphism from $X$ to $Y$, denoting it as $0_{X, Y}$.

\section{Example 3.7}

The category FinVect $\mathbb{F}$ is preadditive because for any $V, W \in \mathrm{ObFinVect}_{\mathbb{F}}, \operatorname{Mor}_{F i n V e c t}(V, W)$ is a class of linear maps equipped with a commutative addition operation (addition of linear maps) with $0 v, W$ being the linear map $v \mapsto 0 W$. Furthermore, the composition of linear maps is distributive over addition.

\section{Example 3.8}

The category $\operatorname{Mat}(\mathbb{F})$ is preadditive since for any $m, n \in \operatorname{ObMat}(\mathbb{F})$, we have that $\operatorname{Mor} \operatorname{Mat}(\mathbb{F})(m, n)$ is an abelian group with respect to the addition operation with $0_{m, n}$ being the zero $n \times m$ matrix. The distributive property then follows from the distributive property of matrices.

Definition 3.9 (Category of rings, category of idempotented rings)

Let Ring denote the category of rings, where the objects are rings and the morphisms are ring homomorphisms. We let Ring $\perp$ denote the category of idempotented rings. In this case, the objects of Ring $\perp$ are idempotented rings and $\operatorname{Mor}_{\operatorname{Ring}}((R, I),(S, J))$ consists of homomorphisms $h: R \rightarrow S$ such that $h(e) \in J$ for all $e \in I$.

Definition 3.10 (Functor)

Suppose $C$ and $\mathcal{D}$ are categories. A functor $F$ from $C$ to $\mathcal{D}$ (written as $\mathrm{F}: C \rightarrow \mathcal{D}$ ) consists of the following:

- $F$ maps every object $X \in \mathrm{Ob} C$ to an object $F(X) \in \mathrm{Ob} \mathcal{D}$ and every morphism $f: X \rightarrow Y$ to a morphism $F(f): F(X) \rightarrow F(Y) \in \operatorname{Mor} \mathcal{D}(F(X), F(Y))$.

- (Preservation of identity) For any $X \in \mathrm{Ob} C, F\left(\mathrm{id}_{X}\right)=\mathrm{id}_{F(X)} \in \mathrm{Ob} \mathcal{D}$.

- (Preservation of composition) Given $f \in \operatorname{Mor}_{C}(X, Y)$ and $g \in \operatorname{Mor}_{C}(Y, Z)$, $F(g \circ f)=F(g) \circ F(f) \in \operatorname{Mor} \mathfrak{D}(F(X), F(Z))$.

Example 3.11 (Identity functor)

Just as there exists an identity mapping for any set, there exists an identity functor $\operatorname{id} C: C \rightarrow C$ for any category $C$ such that $\operatorname{id} C(X)=X$ for all $X \in \mathrm{Ob} C$ and $\operatorname{id} C(f)=f$ for all $f \in \operatorname{Mor} C(X, Y)$.

Example 3.12 (Double dual functor)

An example of a functor from linear algebra is the double dual functor $F:$ FinVect $\mathbb{F}_{F} \rightarrow$ FinVect $F$. For $V \in \mathrm{ObFinVect}_{\mathbb{F}}, F(V)=V^{* *}$, and for $f \in \operatorname{Mor}_{\text {FinVect }}(V, W), F(f)=f^{* *}: V^{* *} \rightarrow W^{* *}$. That is, $F$ maps a vector space to its double dual and a linear map to its double transpose. It is straightforward to verify that $F$ is a functor. 
Definition 3.13 (Additive functor)

Suppose $C$ and $\mathcal{D}$ are preadditive categories. A functor $F: C \rightarrow \mathcal{D}$ is additive if for all $X, Y \in \mathrm{Ob} C, F: \operatorname{Mor} C(X, Y) \rightarrow \operatorname{Mor} \mathcal{D}(F(X), F(Y))$ has the structure of a group homomorphism with respect to addition. That is for any $f, g \in \operatorname{Mor}_{C}(X, Y), F(f+g)=F(f)+F(g) \in \operatorname{Mor} \mathcal{D}(F(X), F(Y))$

Definition 3.14 (Category of small preadditive categories with one object)

Let PreaddCat ${ }_{1}$ denote the category of small preadditive categories with one object. As the name suggests, the objects are small preadditive categories with one object and $\operatorname{Mor}_{\operatorname{PreaddCat}}(C, \mathcal{D})$ is the class of additive functors from $C$ to $\mathcal{D}$.

Definition 3.15 (Category of small preadditive categories with finitely many objects)

Let PreaddCatFin denote the category of small preadditive categories with finitely many objects. The objects are small preadditive categories with finitely many objects and $\operatorname{Mor}_{\operatorname{PreaddCat}}(C, \mathcal{D})$ is the class of additive functors from $C$ to $\mathcal{D}$.

Definition 3.16 (Full functor, faithful functor)

Let $C$ and $\mathcal{D}$ be categories. Recall that a functor $F: C \rightarrow \mathcal{D}$ gives rise to map $F_{X, Y}: \operatorname{Mor} C(X, Y) \rightarrow$ $\operatorname{Mor} \mathcal{D}(F(X), F(Y))$, for all $X, Y \in \mathrm{Ob} C$. The functor $F$ is full on morphisms if $F_{X, Y}$ is surjective and faithful if $F_{X, Y}$ is injective for all $X, Y \in \mathrm{ObC}$. If $F$ is both full and faithful, it is called fully faithful.

Definition 3.17 (Essentially surjective)

Suppose and $C$ and $\mathcal{D}$ are categories. A functor $F: C \rightarrow \mathcal{D}$ is essentially surjective on objects if for all $Y \in \mathrm{Ob} \mathcal{D}$, there exists a $X \in \mathrm{Ob} C$ such that $F(X) \cong Y$.

Definition 3.18 (Natural transformation, natural isomorphism)

Suppose $C$ and $\mathcal{D}$ are categories and $F, G: C \rightarrow \mathcal{D}$ functors. A natural transformation $\eta$ from $F$ to $G$, denoted as $\eta$ : $F \Rightarrow G$, is a mapping from $\mathrm{Ob} C$ to Mor $\mathcal{D}$ that associates to every $X \in \mathrm{Ob} C$ a morphism $\eta_{X} \in \operatorname{Mor} \mathcal{D}(F(X), G(X))$ such that the following diagram commutes for any $f \in \operatorname{Mor}_{C}(X, Y)$.

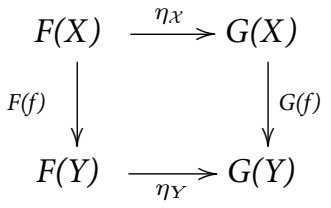

If $\eta X: F(X) \rightarrow G(X)$ is an isomorphism for all $X \in \mathrm{Ob} C$, then $\eta$ is a natural isomorphism.

Example 3.19 (Identity natural transformation)

Let $C, \mathcal{D}$ be categories and $F: C \rightarrow \mathcal{D}$ a functor. The identity natural transformation on $F, \operatorname{id}_{F}: F \Rightarrow F$, is the map $X \mapsto \operatorname{id}_{F(X)} \in \operatorname{Mor} \mathfrak{D}(F(X), F(X))$ for all $X \in \mathrm{ObC}$.

\section{Example 3.20}

Let $\operatorname{id}_{\text {FinVect }}:$ FinVect $_{\mathbb{F}} \rightarrow$ FinVect $_{\mathbb{F}}$ be the identity functor and recall the double dual functor $F:$ FinVect $\mathbb{F} \rightarrow$ FinVect $\mathbb{F}$ from Example 3.12. We define a natural isomorphism $\eta:$ id $_{\text {FinVect }} \Rightarrow F$ like so: for any $V \in \mathrm{Ob}$ FinVect $\mathbb{F}, \eta V \in \operatorname{Mor}_{F_{i n V e c t}}\left(V, V^{* *}\right)$, where $\eta_{V}$ is the isomorphism defined as $\eta_{V}(v): V^{*} \rightarrow \mathbb{F}$ for all $v \in V$ and where $\eta_{V}(v)(f)=f(v)$, for all $f \in V^{*}$. It is then straightforward to check that the following diagram commutes for any $V, W \in$ Ob FinVect $\mathbb{F}$ and $f: V \rightarrow W$.

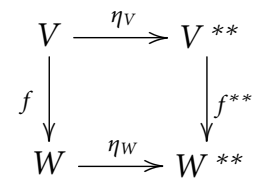


Definition 3.21 (Equivalence of categories)

Two categories $C$ and $\mathcal{D}$ are equivalent (written $C \simeq \mathcal{D}$ ) if there exists a pair of functors $F: C \rightarrow \mathcal{D}$, $G: \mathcal{D} \rightarrow C$ and a pair of natural isomorphisms $\eta: \operatorname{id}_{C} \Rightarrow G \circ F, \epsilon: \operatorname{id} \mathcal{D} \Rightarrow F \circ G$.

Lemma 3.22 $2^{12}$ Suppose $C$ and $\mathcal{D}$ are categories and $F: C \rightarrow \mathcal{D}$ a functor. The functor $F$ yields an equivalence of categories if and only if $F$ is fully faithful on morphisms and essentially surjective on objects.

\section{Example 3.23}

Recall the category of matrices $\operatorname{Mat}(\mathbb{F})$ from Example 3.8 and the category of finite-dimensional vector spaces FinVect $\mathbb{F}$ from Example 3.7. Consider the functor $F: \operatorname{Mat}(\mathbb{F}) \rightarrow$ FinVect $\mathbb{F}$ that maps any $n \in \mathrm{ObMat}(\mathbb{F})$ to $\mathbb{F}^{n}$ and any matrix to its corresponding linear map with respect to the standard bases. Then for any $V \in \mathrm{ObFinVect} \mathbb{F}$, there exists a $n \in \mathrm{ObMat}(\mathbb{F})$ such that $\operatorname{dim}\left(\mathbb{F}^{n}\right)=\operatorname{dim}(V)$ and so $F(n)=\mathbb{F}^{n} \cong V$. Thus $F$ is essentially surjective on objects and fully faithful on morphisms by basic results in linear algebra on the correspondences between linear maps and matrices, and so $\operatorname{Mat}(\mathbb{F}) \simeq \operatorname{FinVect} \mathbb{F}$.

Definition 3.24 (Adjoint functors)

Let $C$ and $\mathcal{D}$ be categories and $H: C \rightarrow \mathcal{D}, J: D \rightarrow C$ functors. The functor $H$ is left adjoint to $J$, denoted $H \dashv J$, if there exist natural transformations $\eta: \operatorname{id}_{C} \Rightarrow J \circ H$ and $\epsilon: H \circ J \Rightarrow \operatorname{id} \mathcal{D}$, such that the following diagrams commute for any $X \in \mathrm{ObC}$ and $Y \in \mathrm{ObD}$.
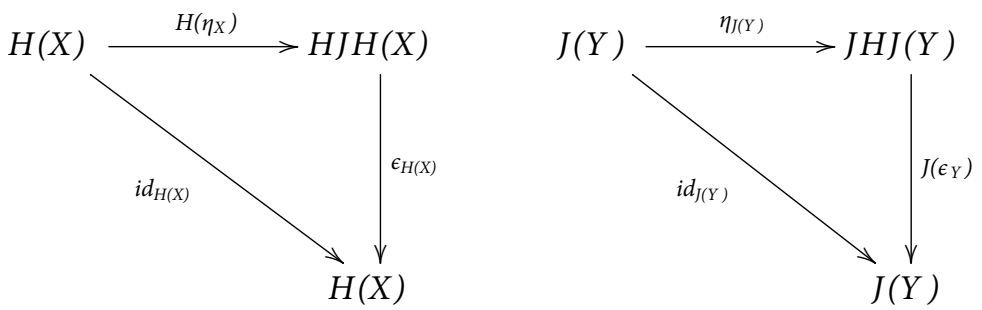

The commutative diagrams above are referred to as the triangle identities. 


\section{HIGHER CATEGORY THEORY}

In this section, we extend some earlier definitions from category theory into higher category theory, namely categories and functors. We will show how the earlier examples of the category of rings and category of idempotented rings can be enhanced to 2-categories. Lastly, the definition of an equivalence of 2-categories is given.

Definition 4.1 (Strict 2-category)

A strict 2-category $C$ is a category where for every $X, Y \in \operatorname{Ob} C, \operatorname{Mor} C(X, Y)$ is a category whose objects are the morphisms from $X$ to $Y$ together with the axioms below. To avoid confusion, we will refer to morphisms between objects of $C$ as 1-morphisms and denote their composition with the usual $\circ$ and morphisms between objects of $\operatorname{Mor} C(X, Y)$ as 2-morphisms for all $X, Y \in \mathrm{Ob} C$. We will write $\alpha: f \Rightarrow g$ to denote that $\alpha$ is a 2-morphism from $\operatorname{Mor}_{M_{C}}(X, Y)(f, g)$.

- (Vertical composition) Let $f, g, h \in \operatorname{Ob} \operatorname{Mor} C(X, Y)$. Composition in the category $\operatorname{Mor} C(X, Y)$ is called vertical composition and is denoted as ${ }^{\circ}$. That is, for 2-morphisms $\alpha: f \Rightarrow g$ and $\beta: g$ $\Rightarrow h$, there is a 2-morphism $\beta \circ_{v} \alpha: f \Rightarrow h$. Furthermore, this vertical composition is associative.

- (Horizontal composition) Let $f, g \in \operatorname{Ob}_{\operatorname{Mor}}(X, Y), f^{\prime}, g^{\prime} \in \operatorname{Ob}_{\operatorname{Mor}}(Y, Z)$. There is a map of horizontal composition

$$
\operatorname{Mor}_{M_{C}}(Y, Z)\left(f^{\prime}, g^{\prime}\right) \times \operatorname{Mor}_{M_{C}}(X, Y)(f, g) \rightarrow \operatorname{Mor}_{M_{C}}(X, Z)\left(f^{\prime} \circ f, g^{\prime} \circ g\right)
$$

and we denote this composition as $\circ$. That is, for 2-morphisms $\alpha: f \Rightarrow g$ and $\alpha^{\prime}: f^{\prime} \Rightarrow g^{\prime}$, there is a 2-morphism $\alpha^{\prime} \circ h \alpha: f^{\prime} \circ f \Rightarrow g^{\prime} \circ \mathrm{g}$. Furthermore, this horizontal composition is associative.

- (Interchange law) Let $f, g, h \in \operatorname{Ob} \operatorname{Mor} C(X, Y), f^{\prime}, g^{\prime}, h^{\prime} \in \operatorname{Ob} \operatorname{Mor} C(Y, Z)$. Given 2-morphisms $\alpha: f \Rightarrow g, \beta: g \Rightarrow h, \alpha^{\prime}: f^{\prime} \Rightarrow g^{\prime}$ and $\beta^{\prime}: g^{\prime} \Rightarrow h^{\prime},\left(\beta^{\prime} \circ_{\nu} \alpha^{\prime}\right) \circ h\left(\beta \circ_{\nu} \alpha\right)=\left(\beta^{\prime} \circ h \beta \circ_{\nu}\left(\alpha^{\prime} \circ h \alpha\right)\right.$.

- (Identity) For any $X \in \mathrm{Ob} C$, there exists an identity 1-morphism id $X$ and an identity 2-morphism $\operatorname{id}_{i_{X}}: \operatorname{id}_{X} \Rightarrow \operatorname{id}_{X}$. The 2-morphism $\operatorname{id}_{i_{X}}$ serves as an identity for both vertical and horizontal composition. That is, for any $\alpha: \operatorname{id}_{X} \Rightarrow \operatorname{id}_{X}, \operatorname{id}_{X}{ }^{\circ} \gamma \alpha=\alpha=\alpha \circ_{v} \operatorname{id} X$. For any 2-morphism $\beta: f \Rightarrow g$ where $f$ and $g$ are 1-morphisms in $\operatorname{Mor}_{C}(\mathrm{X}, \mathrm{Y}), \beta \circ h \operatorname{idid}_{X}=\beta=\operatorname{idid}_{Y} \circ h \beta$.

Remark 4.2 A 2-category differs from a strict 2-category in that it does not require the composition of 1-morphisms to be associative. Instead, we require that $h \circ(g \circ f) \cong(h \circ g) \circ f$ for composable 1-morphisms $h, g$, and $f$. Throughout this document, we will assume that all 2 -categories are strict and so we will omit the term "strict" and refer to them just as 2-categories.

Example 4.3 (Category of small categories)

The category of small categories is an example of a 2-category and we will simply denote it by Cat. As the name suggests, objects of Cat are small categories and the morphisms between these categories are functors. It is straightforward to verify that Cat is truly a category since the axioms of a functor imply the domain, codomain, identity, composition and associativity axioms of a morphism in a category. Then Cat can be enhanced to a 2-category with the 2-morphisms being natural transformations.

Proposition 4.4 (Category of small categories)

Recall the category of rings, Ring, whose objects are rings and whose morphisms are homomorphisms. Then Ring can be enhanced to a 2-category with 2-morphisms being defined as follows: For rings $R, S$ and homomorphisms $f: R \rightarrow S$ and $g: R \rightarrow S$, a 2-morphism $\alpha: f \Rightarrow g$ is an element $\alpha \in S$ such that $\alpha f(r)=g(r) \alpha$ for all $r \in R$. 
Proof.

- (Vertical composition) Let $R, S \in \mathrm{Ob} \operatorname{Ring}$ and $f, g, h \in \operatorname{Mor} \operatorname{Ring}(R, S)$. Given 2-morphisms $\alpha: f \Rightarrow g$ and $\beta: g \Rightarrow h$, we want to show that there exists the vertical composition $\beta \circ_{\nu} \alpha$ such that $\left(\beta \circ_{\nu} \alpha\right) f(r)=g(r)\left(\beta \circ_{v} \alpha\right)$ for all $r \in R$. In this case, vertical composition corresponds to the multiplication operation - in $S$, so we will often abuse notation and omit ${ }_{\nu}$. Note that associativity of ${ }^{\circ} v$ follows from the associativity of multiplication in a ring. Since $\alpha$ and $\beta$ are 2-morphisms, our definition tells us that $\alpha f(r)=g(r) \alpha$ and $\beta g(r)=h(r) \beta$ for all $r \in R$.

Combining these two facts, we get:

$$
\begin{gathered}
\alpha f(r)=g(r) \alpha \\
\Longrightarrow \beta \alpha f(r)=\beta g(r) \alpha \\
\Longrightarrow \beta \alpha f(r)=h(r) \beta \alpha
\end{gathered}
$$

So $\beta \alpha$ is precisely an element of $S$ such that $\beta \alpha f(r)=h(r) \beta \alpha$ for all $r \in R$.

- (Horizontal composition) Let $R, S, T \in \mathrm{ObRing}, f, g \in \operatorname{Mor} \operatorname{Ring}(R, S)$ and $f^{\prime}, g^{\prime} \in \operatorname{Mor} \operatorname{Ring}(S, T)$. Given 2-morphisms $\alpha: f \Rightarrow g$ and $\alpha^{\prime}: f^{\prime} \Rightarrow g^{\prime}$, we define the horizontal composition $\alpha^{\prime} \circ h \alpha$ to be the element $\alpha^{\prime} f^{\prime}(\alpha)$ in T. This leads to the following equalities:

$$
\begin{gathered}
\alpha f(r)=g(r) \alpha \\
\Longrightarrow f^{\prime}(\alpha f(r))=f^{\prime}(g(r) \alpha) \\
\Longrightarrow f^{\prime}(\alpha) f^{\prime}(f(r))=f^{\prime}(g(r)) f^{\prime}(\alpha) \\
\Longrightarrow \alpha^{\prime} f^{\prime}(\alpha) f^{\prime}(f(r))=\alpha^{\prime} f^{\prime}(g(r)) f^{\prime}(\alpha) \\
\Longrightarrow \alpha^{\prime} f^{\prime}(\alpha) f^{\prime}(f(r))=g^{\prime}(g(r)) \alpha^{\prime} f^{\prime}(\alpha)
\end{gathered}
$$

Given another 2-morphism $\alpha^{\prime \prime}: f^{\prime \prime} \Rightarrow g^{\prime \prime}$ where $f^{\prime \prime}, g^{\prime \prime} \in \operatorname{Mor} \operatorname{Ring}(T, U)$, we have:

$$
\begin{aligned}
\left(\alpha^{\prime \prime} \circ h \alpha\right) \circ h \alpha & =\left(\alpha^{\prime \prime} f^{\prime \prime}\left(\alpha^{\prime}\right)\right) \circ h \alpha \\
& =\alpha^{\prime \prime} f^{\prime \prime}\left(\alpha^{\prime}\right) f^{\prime \prime} f^{\prime}(\alpha) \\
& =\alpha^{\prime \prime}\left(f^{\prime \prime}\left(\alpha^{\prime} f^{\prime}(\alpha)\right)\right. \\
& =\alpha^{\prime \prime}\left(f^{\prime \prime}\left(\alpha^{\prime} \circ h \alpha\right)\right) \\
& =\alpha^{\prime \prime} \circ h\left(\alpha^{\prime} \circ h \alpha\right)
\end{aligned}
$$

and so horizontal composition is associative.

- (Interchange law) Let $R, S, T \in \mathrm{Ob}$ Ring with 1-morphisms $f, g, h \in \operatorname{Mor} \operatorname{Ring}(R, S), f^{\prime}, g^{\prime}, h^{\prime} \in$ MorRing $(S, T)$. Given 2-morphisms $\alpha: f \Rightarrow g, \beta: g \Rightarrow h, \alpha^{\prime}: f^{\prime} \Rightarrow g^{\prime}$ and $\beta^{\prime}: g^{\prime} \Rightarrow h^{\prime}$ we have the following equalities:

$$
\begin{gathered}
\left(\beta^{\prime} \alpha^{\prime}\right) \circ h(\beta \alpha)=\beta^{\prime} \alpha^{\prime} f^{\prime}(\beta \alpha), \\
\beta^{\prime} \circ h \beta=\beta^{\prime} g^{\prime}(\beta), \\
\alpha^{\prime} \circ h \alpha=\alpha^{\prime} f^{\prime}(\alpha) .
\end{gathered}
$$

Then we know that:

$$
\begin{aligned}
\left(\beta^{\prime} \circ h \beta\right) \circ_{v}\left(\alpha^{\prime} \circ h \alpha\right) & =\beta^{\prime} g^{\prime}(\beta) \alpha^{\prime} f^{\prime}(\alpha) \\
& =\beta^{\prime} \alpha^{\prime} f^{\prime}(\beta) f^{\prime}(\alpha) \\
& =\beta^{\prime} \alpha^{\prime} f^{\prime}(\beta \alpha) \\
& =\left(\beta^{\prime} \circ \circ_{v} \alpha^{\prime}\right) \circ h\left(\beta \circ_{v} \alpha\right)
\end{aligned}
$$


- (Identity) Assume that $R, S \in \mathrm{Ob}$ Ring with 1-morphisms $f, g \in \operatorname{MorRing}(R, S)$ and $\operatorname{id}_{R} \in$ $\operatorname{Mor}_{\text {Ring }}(R, R)$. We claim that the multiplicative ring identity $1_{R}: \operatorname{id}_{R} \Rightarrow \mathrm{id}_{R}$ is an identity for both vertical composition from $\operatorname{id}_{R}$ to $\mathrm{id}_{R}$ and horizontal composition from $R$ to $S$. Recall that vertical composition corresponds to ring multiplication, so for all $\alpha: \operatorname{id}_{R} \Rightarrow \operatorname{id}_{R}, 1_{R} \circ_{\nu} \alpha=$ $\alpha=\alpha \circ_{v} 1_{R}$. Moreover, for all $\beta: f \Rightarrow g$,

$$
\beta \circ h 1_{R}=\beta \circ_{\nu} f\left(1_{R}\right)=\beta=1_{S} \circ_{\nu} \operatorname{id} S(\beta)=1 S \circ h \beta .
$$

\section{Proposition 4.5}

Recall the category Ring $\perp$ whose objects are idempotented rings and whose morphisms are ring homomorphisms that respect the complete multiset of orthogonal idempotents. We claim that Ring $\perp$ is a 2-category with 2-morphisms being defined as follows. Let $(R, I),(S, J) \in \mathrm{Ob}$ Ring $\perp$ and 1-morphisms $f, g \in \operatorname{Mor}_{\text {Ring }}((R, I),(S, J))$. A 2-morphism $\alpha: f \Rightarrow g$ in Ring $\perp$ is a function $\alpha: I \rightarrow S$ such that for any $e$ in $I, \alpha(e)$ is an element of $g(e) S f(e) \subseteq S$ and for any $r \in R$ and $e^{\prime} \in I, \alpha\left(e^{\prime}\right) f\left(e^{\prime} r e\right)=g\left(e^{\prime} r e\right) \alpha(e)$.

Proof.

- (Vertical composition) Let $(R, I),(S, J) \in \mathrm{Ob}$ Ring $\perp$ with 1-morphisms

$$
f, g, \mathrm{~h} \in \operatorname{MorRing}_{\perp}((R, I),(S, J)) .
$$

Given 2-morphisms $\alpha: f \Rightarrow g$ and $\beta: g \Rightarrow h$, we define $\beta \circ_{\nu} \alpha$ to be the function from $I$ to $S$ such that for any $e \in I, \beta \circ_{v} \alpha(e)=\beta(e) \cdot \alpha(e)$ where $\cdot$ is the usual multiplication in the ring $S$, which we will sometimes omit. Since $\alpha\left(e^{\prime}\right) f\left(e^{\prime} r e\right)=g\left(e^{\prime} r e\right) \alpha(e)$ and $\beta\left(e^{\prime}\right) g\left(e^{\prime} r e\right)=h\left(e^{\prime} r e\right) \beta(e)$, the equalities below hold.

$$
\begin{gathered}
\alpha\left(e^{\prime}\right) f\left(e^{\prime} r e\right)=g\left(e^{\prime} r e\right) \alpha(e) \\
\Longrightarrow \beta\left(e^{\prime}\right) \alpha\left(e^{\prime}\right) f\left(e^{\prime} r e\right)=\beta\left(e^{\prime}\right) g\left(e^{\prime} r e\right) \alpha(e) \\
\Longrightarrow \beta\left(e^{\prime}\right) \alpha\left(e^{\prime}\right) f\left(e^{\prime} r e\right)=h\left(e^{\prime} r e\right) \beta(e) \alpha(e)
\end{gathered}
$$

So $\beta \circ_{v} \alpha$ is indeed a 2-morphism from $f$ to $h$. Furthermore, when given another 2-morphism $\gamma: h \Rightarrow j$, where $j \in \operatorname{Mor}_{\text {Ring }_{\perp}}((R, I),(S, J))$, vertical composition is associative since

$$
\begin{aligned}
\left(\left(\gamma \circ{ }_{\nu} \beta\right) \circ_{\nu} \alpha\right)(e) & =\left(\gamma \circ_{\nu} \beta\right)(e) \alpha(e) \\
& =\gamma(e) \beta(e) \alpha(e) \\
& =\gamma(e) \circ\left(\beta \circ_{\nu} \alpha\right)(e) \\
& =\left(\gamma \circ_{\nu}\left(\beta \circ_{\nu} \alpha\right)\right)(e) .
\end{aligned}
$$

- (Horizontal composition) Let $(R, I),(S, J),(T, K) \in \mathrm{Ob}_{\text {Ring }} \perp$ with $f, g,:(R, I) \rightarrow(S, J)$ and $f^{\prime}, g^{\prime}:(S, J) \rightarrow(T, K)$. Given 2 morphisms $\alpha: f \Rightarrow g$ and $\alpha^{\prime}: f^{\prime} \Rightarrow g^{\prime}$, we define $\alpha^{\prime} \circ h \alpha$ to be the following function from $I$ to $T$ : For any $e \in I, \alpha^{\prime} \circ h \alpha(e)=\alpha^{\prime}(g(e)) f^{\prime}(\alpha(e))$. Then we have the following equalities:

$$
\begin{aligned}
\alpha\left(e^{\prime}\right) f\left(e^{\prime} r e\right) & =g\left(e^{\prime} r e\right) \alpha(e) \\
\Longrightarrow f^{\prime}\left(\alpha\left(e^{\prime}\right) f\left(e^{\prime} r e\right)\right) & =f^{\prime}\left(g\left(e^{\prime} r e\right) \alpha(e)\right) \\
\Longrightarrow f^{\prime}\left(\alpha\left(e^{\prime}\right)\right) f^{\prime}\left(f\left(e^{\prime} r e\right)\right) & =f^{\prime}\left(g\left(e^{\prime} r e\right)\right) f^{\prime}(\alpha(e)) \\
\Longrightarrow \alpha^{\prime}\left(g\left(e^{\prime}\right)\right) f^{\prime}\left(\alpha\left(e^{\prime}\right)\right) f^{\prime}\left(f\left(e^{\prime} r e\right)\right) & =\alpha^{\prime}\left(g\left(e^{\prime}\right)\right) f^{\prime}\left(g\left(e^{\prime} r e\right)\right) f^{\prime}(\alpha(e)) \\
\Longrightarrow \alpha^{\prime}\left(g\left(e^{\prime}\right)\right) f^{\prime}\left(\alpha\left(e^{\prime}\right)\right) f^{\prime}\left(f\left(e^{\prime} r e\right)\right) & =g^{\prime}\left(g\left(e^{\prime} r e\right)\right) \alpha^{\prime}(g(e)) f^{\prime}(\alpha(e))
\end{aligned}
$$


and so $\beta \circ h \alpha$ is indeed a 2-morphism from $f^{\prime} \circ f$ to $g^{\prime} \circ g$. Given another object $(U, L)$ in Ring $\perp$ with 1-morphisms $f^{\prime \prime}, g^{\prime \prime} \in \operatorname{Mor}_{\text {Ring }}((T, K),(U, L))$ and 2-morphism $\alpha^{\prime \prime}: f^{\prime \prime} \Rightarrow g^{\prime \prime}$, we have that for any $e \in I$,

$$
\begin{aligned}
\left(\alpha^{\prime \prime} \circ h\left(\alpha^{\prime} \circ h \alpha\right)\right)(e) & =\alpha^{\prime \prime}\left(g^{\prime} g(e)\right) f^{\prime \prime}\left(\left(\alpha^{\prime} \circ h \alpha\right)(e)\right) \\
& =\alpha^{\prime \prime}\left(g^{\prime} g(e)\right) f^{\prime \prime}\left(\alpha^{\prime}\left((g(e)) f^{\prime}(\alpha(e))\right)\right. \\
& =\alpha^{\prime \prime}\left(g^{\prime} g(e)\right) f^{\prime \prime}\left(\alpha^{\prime}(g(e))\right) f^{\prime \prime} f(\alpha(e)) \\
& =\left(\alpha^{\prime \prime} \circ \alpha^{\prime}(g(e)) f^{\prime \prime} f(\alpha(e))\right. \\
& =\left(\left(\alpha^{\prime \prime} \circ{ }^{\prime} \alpha^{\prime}\right) \circ \alpha\right)(e)
\end{aligned}
$$

and so this horizontal composition is associative.

- (Interchange law) Suppose $(R, I),(S, J),(T, K) \in O$ Ob Ring $\perp$ with 1-morphisms $f, g, h \in$ $\operatorname{Mor}_{\text {Ring }}((R, I),(S, J)), f^{\prime}, g^{\prime}, h^{\prime} \in \operatorname{Mor}_{\text {Ring }}((S, J),(T, K))$. Let $\alpha: f \Rightarrow g, \beta: g \Rightarrow h, \alpha^{\prime}: f^{\prime} \Rightarrow g^{\prime}$ and $\beta^{\prime}: g^{\prime} \Rightarrow h^{\prime}$ be 2-morphisms. We know that $\beta(e) \in h(e) S g(e) \subseteq S$ and that $g(e)$ and $h(e)$ are in $J$. Then for any idempotent $h(e) \in T$, there exists an element $\alpha^{\prime}(g(e)) \in T$ such that $\alpha^{\prime}(h(e)) f^{\prime}(h(e)$ $s g(e))=g^{\prime}(h(e) s g(e)) \alpha^{\prime}(g(e))$. If we let that $h(e) s g(e)=\beta(e)$, we get that $\alpha^{\prime}(h(e)) f^{\prime}(\beta(e))=g^{\prime}(\beta(e))$ $\alpha^{\prime}(g(e))$. Thus,

$$
\begin{aligned}
\left(\left(\beta^{\prime} \circ h \beta\right) \circ_{v}\left(\alpha^{\prime} \circ h \alpha\right)\right)(e) & =\left(\beta^{\prime} \circ h \beta\right)(e) \circ_{v}\left(\alpha^{\prime} \circ h \alpha\right)(e) \\
& =\beta^{\prime}(h(e)) g^{\prime}(\beta(e)) \circ_{\nu} \alpha^{\prime}(g(e)) f^{\prime}(\alpha(e)) \\
& =\beta^{\prime}(h(e)) g^{\prime}(\beta(e)) \alpha^{\prime}(g(e)) f^{\prime}(\alpha(e)) \\
& =\beta^{\prime}(h(e)) \alpha^{\prime}(h(e)) f^{\prime}(\beta(e)) f^{\prime}(\alpha(e)) \\
& =\left(\beta^{\prime} \circ_{v} \alpha^{\prime}\right)(h(e)) f^{\prime}\left(\left(\beta \circ_{v} \alpha\right)(e)\right) \\
& =\left(\left(\beta^{\prime} \circ_{v} \alpha^{\prime}\right) \circ_{h}\left(\beta \circ_{v} \alpha\right)\right)(e) .
\end{aligned}
$$

- (Identity) Let $(R, I) \in \mathrm{Ob} \operatorname{Ring} \perp$ with $f, g \in \operatorname{Mor}_{\operatorname{Ring}}((R, I),(S, J))$ and $\operatorname{id}_{R} \in \operatorname{Mor}_{\operatorname{Ring}}((R, I)$, $(R, I)$ ), the identity homomorphism from $R$ to $R$. We define $\operatorname{id}_{\mathrm{id}_{R}}: \mathrm{id}_{R} \Rightarrow \mathrm{id}_{R}$ to be the inclusion map of $I$ into $R$. Then for any $\alpha: \operatorname{id}_{R} \Rightarrow \operatorname{id}_{R}$ and $e \in I, \alpha(e)=e r e \in e R e$ for some $r \in R$, and so

$\left(\alpha \circ{ }_{\nu} \operatorname{idid}_{R}\right)(e)=\alpha(e) \operatorname{idid}_{R}(e)=$ eree $=$ ere $=$ eere

$$
=e \alpha(e)=\operatorname{idid}_{R}(e) \alpha(e)=\left(\operatorname{idid}_{R}{ }^{\circ} \alpha\right)(e) .
$$

Furthermore, let $(S, J) \in \mathrm{Ob} \operatorname{Ring} \perp$ with $f, g \in \operatorname{MorRing}_{\perp}((R, I),(S, J))$ and $\beta: f \Rightarrow g$. For any $e \in I, \beta(e)$ $=g(e) s f(e) \in g(e) S f(e)$ for some $s \in S$. Then we have the following:

$$
\begin{aligned}
\left(\beta \circ h \operatorname{idid}_{R}\right)(e)=\beta\left(\operatorname{id}_{R}(e)\right) f\left(\operatorname{id}_{\operatorname{id}_{R}}(e)\right)=g(e) s f(e) f(e)=g(e) s f(e) & =\beta(e) \\
=g(e) g(e) s f(e) & =\operatorname{idid}_{S}(g(e)) \operatorname{id}_{S}(\beta(e))=\left(\operatorname{idid}_{S} \circ h \beta\right)(e)
\end{aligned}
$$


Definition 4.6 (2-functor)

Suppose $C$ and $D$ are 2-categories. A map $F: C \rightarrow \mathcal{D}$ is a 2 -functor if it satisfies the following properties:

- $F$ maps every object $X \in C$ to an object $F(X) \in \mathcal{D}$, every 1-morphism $f \in \operatorname{Mor}_{C}(X, Y)$ to a 1-morphism $F(f) \in \operatorname{Mor} \mathcal{D}(F(X), F(Y))$ and every 2-morphism $\alpha: f \Rightarrow g$ to a 2-morphism $F(\alpha): F(f) \Rightarrow F(g)$.

- (Preservation of identity) For any $X \in \operatorname{Ob} C, F\left(\operatorname{id}_{X}\right)=\operatorname{id}_{F(X)} \in \operatorname{Mor} \mathcal{D}(F(X), F(X))$. For any $\operatorname{id}_{i_{X}}: \operatorname{id}_{X} \Rightarrow \operatorname{id}_{X}, F\left(\operatorname{id}_{i_{X}}\right)=\operatorname{id}_{i d_{F(X)}}, \operatorname{id}_{i d_{F(X)}}: \operatorname{id}_{F(X)} \Rightarrow \operatorname{id}_{F(X)}$.

- (Preservation of composition) Given $f \in \operatorname{Mor}_{C}(X, Y)$ and $g \in \operatorname{Mor}_{C}(Y, Z), F(g \circ f)=F(g) \circ F(f)$ $\in \operatorname{Mor} \mathcal{D}(F(X), F(Z))$.

- (Preservation of vertical composition) Given 1-morphisms $f, g, h \in \operatorname{Mor}_{C}(X, Y)$ and 2-morphisms $\alpha: f \Rightarrow g$ and $\beta: g \Rightarrow h, F\left(\beta \circ_{\nu} \alpha\right)=F(\beta) \circ_{\nu} F(\alpha): F(f) \Rightarrow F(h)$.

- (Preservation of horizontal composition) Given 1-morphisms $f, g \in \operatorname{Mor} C(X, Y), f^{\prime}, g^{\prime} \in$ $\operatorname{Mor}_{C}(Y, Z)$ and 2-morphisms $\alpha: f \Rightarrow g, \alpha^{\prime}: f^{\prime} \Rightarrow g^{\prime}, F\left(\alpha^{\prime} \circ h \alpha\right)=F\left(\alpha^{\prime}\right) \circ h F(\alpha): F\left(f^{\prime}\right) \circ F(f) \Rightarrow$ $F\left(g^{\prime}\right) \circ F(g)$.

Definition 4.7 (2-natural transformation)

Let $C$ and $\mathcal{D}$ be 2 -categories with 2-functors $F, G: C \rightarrow \mathcal{D}$. A 2-natural transformation $\alpha$ from $F$ to $G$ denoted as $\alpha: F \Rightarrow G$, is a natural transformation that commutes with the action of $F$ and $G$ on 2-morphisms. That is, for objects $X, Y \in \mathrm{Ob} C$ with a 1 -morphism $f \in \operatorname{Mor}_{C}(X, Y)$, we have a 2-morphism $\alpha_{f}: G(f) \circ \eta_{X} \Rightarrow \eta Y \circ F(f)$ such that the following diagram commutes.

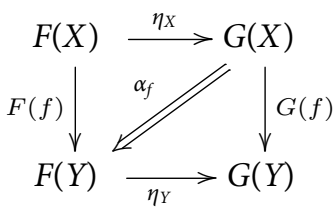

Definition 4.8 (Equivalence of 2-categories)

Suppose $C$ and $\mathcal{D}$ are 2-categories. The categories $C$ and $\mathcal{D}$ are equivalent as 2-categories if there exist 2-functors $F: C \rightarrow \mathcal{D}$ and $G: \mathcal{D} \rightarrow C$ with 2-natural isomorphisms $F \circ G \cong \operatorname{id} \mathcal{D}$ and $G \circ F \cong \operatorname{id}_{C}$.

Lemma 4.9 Let $C$ and $\mathcal{D}$ be 2-categories and $F: C \rightarrow \mathcal{D}$ a 2-functor. Then $F$ yields an equivalence of 2-categories if and only if $F$ is essentially surjective on objects and fully faithful on both 1-morphisms and 2-morphisms.

Proof.

The 2-functor $F$ is fully faithful on 1-morphisms and 2-morphisms if, for all $X, Y \in \mathrm{Ob} C$, it induces an isomorphism of categories $\operatorname{Mor} C(X, Y) \rightarrow \operatorname{Mor} \mathcal{D}(F(X), F(Y))$. Then the statement follows from a more general argument concerning $\mathcal{V}$-encriched categories, ${ }^{13}$ after taking $\mathcal{V}$ to be the category of small categories. 


\section{RINGS AND CATEGORIES}

Many familiar algebraic structures with an algebraic definition also have a category theoretic one. For example, a ring $R$ can be viewed as a preadditive category with one object by defining the ring category associated to the ring $R$ to be the category $C_{R}$ such that $\mathrm{Ob} C_{R}=\{*\}$ and $\operatorname{Mor} C_{R}=R$. So the object of $C_{R}$ is a formal one and the morphisms of $C_{R}$ are elements of $R$. We let the composition and addition of morphisms be the respective multiplication and addition operation in $R$. It follows that the identity and zero morphism are the multiplicative and additive identities respectively in $R$. Likewise, to any preadditive category $C$ with one object *, we can associate the ring $R_{C}=\operatorname{Mor} C(*, *)$. So the multiplication and addition in the ring is the respective composition and addition in the class of morphisms of a category. We complete our definition of $R_{C}$ by letting the additive and multiplicative identities of the ring be the zero and identity morphisms of $\operatorname{Mor} C(*, *)$. From a category theorist's point of view, a ring and a preadditive category with one object are the same thing seen through two different lenses.

Lemma 5.1 If $C$ is a preadditive category, then for all $X \in \operatorname{Ob} C, \operatorname{Mor} C(X, X)$ is a ring.

Proof.

For any $X \in \mathrm{Ob} C, \operatorname{Mor} C(X, X)$ has the structure of an abelian group with respect to addition and the definition of a category gives us the associative composition operation with an identity morphism id $x$. Finally, the axioms of a preadditive category give us the distributive property.

Lemma 5.2 If $C$ and $\mathcal{D}$ are preadditive categories and $F: C \rightarrow \mathcal{D}$ an additive functor, then for all $X \in \operatorname{Ob} C, F_{X}, X: \operatorname{Mor} C(X, X) \rightarrow \operatorname{Mor} \mathfrak{D}(F(X), F(X))$ is a ring homomorphism.

Proof.

- (Preserves identity) The map $F_{X}, X: \operatorname{Mor} C(X, X) \rightarrow \operatorname{Mor} \mathcal{D}(F(X), F(X))$ preserves the identity morphism which we have taken to be the identity element of our ring. Thus we have that $F\left(\operatorname{id}_{X}\right)=\operatorname{id}_{F(X)}$.

- (Preserves products) Recall that we took our multiplication operation to be the composition of morphisms. The axioms of a functor gives us that for all $f, g \in \operatorname{Mor}_{C}(X, X)$, we have that $F_{X, X}(f \circ g)=F_{X, X}(f) \circ F_{X, X}(g)$.

- (Preserves sums) Since $F$ is an additive functor, it acts like a group homomorphism on $\operatorname{Mor}_{C}(X, X)$ with respect to addition. So for $f, g \in \operatorname{Mor}_{C}(X, X), F_{X, X}(f+g)=F_{X, X}(f)+F_{X, X}(g)$. 


\section{Proposition 5.3}

Similar to how we can associate every ring with a preadditive category with one object, we can also associate an idempotented ring with with a category that preserves the idempotent structure. For an idempotented ring $(R, I)$, we can construct a preadditive category $C_{(R, I)}$ where $\mathrm{Ob} C_{(R, I)}=I$ and $\operatorname{Mor}_{(R, I)}\left(e_{i}, e_{j}\right)=\left\{e_{j} R e_{i}=e_{j} r e_{i}: r \in R\right\}$. Composition in the category is just multiplication in the ring and so the identity morphism is $e_{i}$ for any $e_{i} \in \mathrm{Ob} C(R, I)$.

Proof.

- (Identity) For any $e_{j} r e_{i} \in \operatorname{Mor}_{(R, I)}\left(e_{i}, e_{j}\right),\left(e_{j} r e_{i}\right)\left(e_{i}\right)=e_{j} r e_{i} e_{i}=e_{j} r e_{i}=e_{j} e_{j} r e_{i}=\left(e_{j}\right)\left(e_{j} r e_{i}\right)$.

- (Composition) For any $e_{j} r e_{i} \in \operatorname{Mor}_{(R, I)}\left(e_{i}, e_{j}\right), e_{k} s e_{j} \in \operatorname{Mor}_{(R, I)}\left(e_{j}, e_{k}\right),\left(e_{k} r e_{j}\right)\left(e_{j} s e_{i}\right)=e_{k} r e_{j} e_{j} s e_{i}=$ $e_{k r e j s e} \in \operatorname{Mor}_{(R, I)}\left(e_{i}, e_{k}\right)$.

- (Associativity) Since the morphisms of $C_{(R, I)}$ are just elements in $R$, associativity of morphisms follows from the associativity of multiplication in $R$.

- (Abelian group structure) For any $e_{j} r e_{i} \in \operatorname{Mor}_{(R, I)}\left(e_{i}, e_{j}\right)$, we have a morphism $e_{j} r e_{i}+e_{j} s e_{i}=$ $e_{j}(r+s) e_{i}=e_{j}(s+r) e_{i}=e_{j} s e_{i}+e_{j} r e_{i} \in \operatorname{Mor}_{(R, I)}\left(e_{i}, e_{j}\right)$.

- (Left distributive property) For any $e_{j} r e_{i}, e_{j} s e_{i} \in \operatorname{Mor}_{(R, I)}\left(e_{i}, e_{j}\right)$ and $e_{k} t e_{j}, e_{k} u e_{j} \in \operatorname{Mor}_{(R, I)}$ $\left(e_{j}, e_{k}\right)$, we have that:

$$
\begin{aligned}
\left(e_{k} t e_{j}\right)\left(e_{j} r e_{i}+e_{j} s e_{i}\right) & =\left(e_{k} t e_{j}\right)\left(e_{j}(r+s) e_{i}\right) \\
& =\left(e_{k} t e_{j}(r+s) e_{j}\right) \\
& =\left(e_{k} t e_{j} r e_{i}\right)+\left(e_{k} t e_{j} s e_{i}\right) \\
& =\left(e_{k} t e_{j} e_{j} r e_{i}\right)+\left(e_{k} t e_{j} e_{j} s e_{i}\right) \\
& =\left(e_{k} t e_{j}\right)\left(e_{j} r e_{i}\right)+\left(e_{k} t e_{j}\right)\left(e_{j} s e_{i}\right)
\end{aligned}
$$

- (Right distributive property) For any $e_{k} t e_{j}, e_{k} u e_{j} \in \operatorname{Mor} C_{(R, I)}\left(e_{j}, e_{k}\right)$ and $e_{j r} e_{i} \in \operatorname{Mor} C_{(R, I)}\left(e_{i}, e_{j}\right)$,

$$
\begin{aligned}
\left(e_{k} t e_{j}+e_{k} u e_{j}\right)\left(e_{j} r e_{i}\right) & =\left(e_{k}(t+u) e_{j}\right)\left(e_{j} r e_{i}\right) \\
& =\left(e_{k}(t+u) e_{j} e_{j} r e_{i}\right) \\
& =\left(e_{k}(t+u) e_{j} r e_{i}\right) \\
& =\left(e_{k} t e_{j} r e_{i}+e_{k} u e_{j} r e_{i}\right) \\
& =\left(e_{k} t e_{j} r e_{i}\right)+\left(e_{k} u e_{j} r e_{i}\right) \\
& =\left(e_{k} t e_{j}\right)\left(e_{j} r e_{i}\right)+\left(e_{k} u e_{j}\right)\left(e_{j} r e_{i}\right)
\end{aligned}
$$

Remark 5.4 We will denote elements of a direct sum $\oplus_{G \in \Gamma} G$ of abelian groups by formal sums $\sum_{G \in \Gamma} g_{G}$, where $g_{G} \in G$ for all $G \in \Gamma$. By convention, we omit zero summands. For instance, any element $g$ of some $G \in \Gamma$ can be thought of as an element of $\bigoplus G \in \Gamma G$, where all other summands are zero. 
Proposition 5.5 Suppose $C$ is a preadditive category with finitely many objects. Let $R_{C}=\oplus_{X}, Y \in \mathrm{ObC}$ $\operatorname{Mor} C(X, Y)$ and $I_{C}=\left\{\operatorname{id}_{X}: X \in \mathrm{Ob} C\right\}$. Then $\left(R_{C}, I_{C}\right)$ is an idempotented ring with addition and multiplication given by the addition and composition of morphisms.

Proof.

We let addition and multiplication in $R_{C}$ be the component-wise addition of morphisms in $C$ and the composition of morphisms in $C$ respectively. That is:

$$
\begin{gathered}
\sum_{X, Y \in \mathrm{Ob} \mathcal{C}} f_{X, Y}+\sum_{X, Y \in \mathrm{Ob} \mathcal{C}} g_{X, Y}=\sum_{X, Y \in \mathrm{Ob} \mathcal{C}}\left(f_{X, Y}+g_{X, Y}\right) \\
\left(\sum_{Y, Y^{\prime} \in \mathrm{Ob} \mathcal{C}} g_{Y, Y^{\prime}}\right) \cdot\left(\sum_{X, X^{\prime} \in \mathrm{Ob} \mathcal{C}} f_{X, X^{\prime}}\right)=\sum_{X, X^{\prime}, Y^{\prime} \in \mathrm{Ob} \mathcal{C}}\left(g_{X^{\prime}, Y^{\prime}} \circ f_{X, X^{\prime}}\right)
\end{gathered}
$$

(Note that the resulting sum above is direct over $X$ and $Y^{\prime}$ but not over $X^{\prime}$.)

We will define the composition of non-composable morphisms to be zero and the respective additive and multiplicative identities to be:

$$
0_{R_{\mathcal{C}}}=\sum_{X, Y \in \mathrm{Ob} \mathcal{C}} 0_{X, Y}, \quad 1_{R_{\mathcal{C}}}=\sum_{X \in \mathrm{Ob} \mathcal{C}} \mathrm{id}_{X} .
$$

Note that for any $X, Y \in \mathrm{Ob} C, \operatorname{id}_{X} \cdot \operatorname{id}_{X}=\operatorname{id}_{X}$ and $\operatorname{id}_{X} \cdot \operatorname{id}_{Y}=0$ by our definition of multiplication. Thus $I_{C}$ is truly a complete multiset of orthogonal idempotents. We check the remaining axioms of an idempotented ring below.

- (Commutativity of addition)

$$
\begin{aligned}
\sum_{X, Y \in \mathrm{Ob} \mathcal{C}} f_{X, Y}+\sum_{X, Y \in \mathrm{Ob} \mathcal{C}} g_{X, Y}=\sum_{X, Y \in \mathrm{Ob} \mathcal{C}}\left(f_{X, Y}+g_{X, Y}\right) \\
=\sum_{X, Y \in \mathrm{Ob} \mathcal{C}}\left(g_{X, Y}+f_{X, Y}\right)=\sum_{X, Y \in \mathrm{Ob} \mathcal{C}} g_{X, Y}+\sum_{X, Y \in \mathrm{Ob} \mathcal{C}} f_{X, Y}
\end{aligned}
$$

- (Associativity of addition)

$$
\begin{aligned}
\left(\sum_{X, Y \in \mathrm{Ob} \mathcal{C}} f_{X, Y}+\sum_{X, Y \in \mathrm{Ob} \mathcal{C}} g_{X, Y}\right)+\sum_{X, Y \in \mathrm{Ob} \mathcal{C}} h_{X, Y} & =\sum_{X, Y \in \mathrm{Ob} \mathcal{C}}\left(f_{X, Y}+g_{X, Y}\right)+\sum_{X, Y \in \mathrm{Ob} \mathcal{C}} h_{X, Y} \\
& =\sum_{X, Y \in \mathrm{Ob} \mathcal{C}}\left(f_{X, Y}+g_{X, Y}+h_{X, Y}\right) \\
& =\sum_{X, Y \in \mathrm{Ob} \mathcal{C}} f_{X, Y}+\sum_{X, Y \in \mathrm{Ob} \mathcal{C}}\left(g_{X, Y}+h_{X, Y}\right) \\
& =\sum_{X, Y \in \mathrm{Ob} \mathcal{C}} f_{X, Y}+\left(\sum_{X, Y \in \mathrm{Ob} \mathcal{C}} g_{X, Y}+\sum_{X, Y \in \mathrm{Ob} \mathcal{C}} h_{X, Y}\right)
\end{aligned}
$$

\section{AJUR volume 12 | Issue 4 | November 2015}


- (Additive identity)

$$
\begin{aligned}
\sum_{X, Y \in \mathrm{Ob} \mathcal{C}} 0_{X, Y}+\sum_{X, Y \in \mathrm{Ob} \mathcal{C}} f_{X, Y}=\sum_{X, Y \in \mathrm{Ob} \mathcal{C}}\left(0_{X, Y}+f_{X, Y}\right)=\sum_{X, Y \in \mathrm{Ob} \mathcal{C}} f_{X, Y} \\
=\sum_{X, Y \in \mathrm{Ob} \mathcal{C}}\left(f_{X, Y}+0_{X, Y}\right)=\sum_{X, Y \in \mathrm{Ob} \mathcal{C}} f_{X, Y}+\sum_{X, Y \in \mathrm{Ob} \mathcal{C}} 0_{X, Y}
\end{aligned}
$$

- (Additive inverses) The existence of additive inverses follows from the fact that for all $X, Y \in \mathrm{Ob} C, \operatorname{Mor} C(X, Y)$ has the structure of an abelian group.

$$
\begin{array}{r}
\sum_{X, Y \in \mathrm{Ob} \mathcal{C}} f_{X, Y}+\sum_{X, Y \in \mathrm{Ob} \mathcal{C}}\left(-f_{X, Y}\right)=\sum_{X, Y \in \mathrm{Ob} \mathcal{C}}\left(f_{X, Y}-f_{X, Y}\right)=\sum_{X, Y \in \mathrm{Ob} \mathcal{C}} 0_{X, Y}=\sum_{X, Y \in \mathrm{Ob} \mathcal{C}}\left(-f_{X, Y}+f_{X, Y}\right) \\
=\sum_{X, Y \in \mathrm{Ob} \mathcal{C}}\left(-f_{X, Y}\right)+\sum_{X, Y \in \mathrm{Ob} \mathcal{C}} f_{X, Y}
\end{array}
$$

- (Associativity of multiplication)

$$
\begin{aligned}
& \left(\left(\sum_{Z, Z^{\prime} \in \mathrm{Ob} \mathcal{C}} h_{Z, Z^{\prime}}\right) \cdot\left(\sum_{Y, Y^{\prime} \in \mathrm{Ob} \mathcal{C}} g_{Y, Y^{\prime}}\right)\right) \cdot\left(\sum_{X, X^{\prime} \in \mathrm{Ob} \mathcal{C}} f_{X, X^{\prime}}\right) \\
& =\left(\sum_{Y, Y^{\prime}, Z, Z^{\prime} \in \mathrm{Ob} \mathcal{C}}\left(h_{Z, Z^{\prime}} \circ g_{Y, Y^{\prime}}\right)\right) \cdot\left(\sum_{X, X^{\prime} \in \mathrm{Ob} \mathcal{C}} f_{X, X^{\prime}}\right) \\
& =\left(\sum_{Y, Y^{\prime}, Z^{\prime} \in \mathrm{Ob} \mathcal{C}}\left(h_{Y^{\prime}, Z^{\prime}} \circ g_{Y, Y^{\prime}}\right)\right) \cdot\left(\sum_{X, X^{\prime} \in \mathrm{Ob} \mathcal{C}} f_{X, X^{\prime}}\right) \\
& =\left(\sum_{X, X^{\prime}, Y, Y^{\prime}, F \in \mathrm{Ob} \mathcal{C}}\left(h_{Y^{\prime}, Z^{\prime}} \circ g_{Y, Y^{\prime}} \circ f_{X, X^{\prime}}\right)\right) \\
& =\left(\sum_{X, Y^{\prime}, Y, Z^{\prime} \in \mathrm{Ob} \mathcal{C}}\left(h_{Y^{\prime}, Z^{\prime}} \circ g_{Y, Y^{\prime}} \circ f_{X, Y}\right)\right) \\
& =\left(\sum_{Y^{\prime}, Z^{\prime} \in \mathrm{Ob} \mathcal{C}} h_{Y^{\prime}, Z^{\prime}}\right) \cdot\left(\sum_{X, Y, Y^{\prime} \in \mathrm{Ob} \mathcal{C}}\left(g_{Y, Y^{\prime}} \circ f_{X, Y}\right)\right) \\
& =\left(\sum_{Y^{\prime}, Z^{\prime} \in \mathrm{Ob} \mathcal{C}} h_{Y^{\prime}, Z^{\prime}}\right) \cdot\left(\left(\sum_{Y, Y^{\prime} \in \mathrm{Ob} \mathcal{C}} g_{Y, Y^{\prime}}\right) \cdot\left(\sum_{X, Y \in \mathrm{Ob} \mathcal{C}} f_{X, Y}\right)\right) \\
& =\left(\sum_{Z, Z^{\prime} \in \mathrm{Ob} \mathcal{C}} h_{Z, Z^{\prime}}\right) \cdot\left(\left(\sum_{Y, Y^{\prime} \in \mathrm{Ob} \mathcal{C}} g_{Y, Y^{\prime}}\right) \cdot\left(\sum_{X, X^{\prime} \in \mathrm{Ob} \mathcal{C}} f_{X, X^{\prime}}\right)\right)
\end{aligned}
$$


- (Multiplicative identity)

$$
\begin{aligned}
\left(\sum_{X \in \mathrm{Ob} \mathcal{C}} \operatorname{id}_{X}\right) \cdot\left(\sum_{Y, Z \in \mathrm{Ob} \mathcal{C}} f_{Y, Z}\right) & =\sum_{X, Y, Z \in \mathrm{Ob} \mathcal{C}}\left(\operatorname{id}_{X} \circ f_{Y, Z}\right) \\
& =\sum_{Y, Z \in \mathrm{Ob} \mathcal{C}}\left(\operatorname{id}_{Z} \circ f_{Y, Z}\right) \\
& =\sum_{Y, Z \in \mathrm{Ob} \mathcal{C}} f_{Y, Z} \\
& =\sum_{Y, Z \in \mathrm{Ob} \mathcal{C}}\left(f_{Y, Z} \circ \mathrm{id}_{Y}\right) \\
& =\sum_{X, Y, Z \in \mathrm{Ob} \mathcal{C}}\left(f_{Y, Z} \circ \mathrm{id}_{X}\right) \\
& =\left(\sum_{Y, Z \in \mathrm{Ob} \mathcal{C}} f_{Y, Z}\right) \circ\left(\sum_{X \in \mathrm{Ob} \mathcal{C}} \mathrm{id}_{X}\right)
\end{aligned}
$$

- (Left distributive property)

$$
\begin{aligned}
\left(\sum_{Y, Y^{\prime} \in \mathrm{Ob} \mathcal{C}} h_{Y, Y^{\prime}}\right) & \left.\cdot\left(\sum_{X, X^{\prime} \in \mathrm{Ob} \mathcal{C}} g_{X, X^{\prime}}\right)+\left(\sum_{X, X^{\prime} \in \mathrm{Ob} \mathcal{C}} f_{X, X^{\prime}}\right)\right) \\
& =\left(\sum_{Y, Y^{\prime} \in \mathrm{Ob} \mathcal{C}} h_{Y, Y^{\prime}}\right) \cdot\left(\sum_{X, X^{\prime} \in \mathrm{Ob} \mathcal{C}}\left(g_{X, X^{\prime}}+f_{X, X^{\prime}}\right)\right) \\
& =\left(\sum_{X, X^{\prime}, Y, Y^{\prime} \in \mathrm{Ob} \mathcal{C}} h_{Y, Y^{\prime}} \circ\left(g_{X, X^{\prime}}+f_{X, X^{\prime}}\right)\right) \\
& =\left(\sum_{X, X^{\prime}, Y, Y^{\prime} \in \mathrm{Ob} \mathcal{C}}\left(h_{Y, Y^{\prime}} \circ g_{X, X^{\prime}}\right)+\left(h_{Y, Y^{\prime}} \circ f_{X, X^{\prime}}\right)\right) \\
& =\left(\sum_{X, X^{\prime}, Y^{\prime} \in \mathrm{Ob} \mathcal{C}}\left(h_{X^{\prime}, Y^{\prime}} \circ g_{X, X^{\prime}}\right)+\left(h_{X^{\prime}, Y^{\prime}} \circ f_{X, X^{\prime}}\right)\right) \\
& =\left(\sum_{X, X^{\prime}, Y^{\prime} \in \mathrm{Ob} \mathcal{C}}\left(h_{X^{\prime}, Y^{\prime}} \circ g_{X, X^{\prime}}\right)\right)+\left(\sum_{X, X^{\prime}, Y^{\prime} \in \mathrm{Ob} \mathcal{C}}\left(h_{X^{\prime}, Y^{\prime}} \circ f_{X, X^{\prime}}\right)\right) \\
& =\left(\sum_{Y, Y^{\prime}} h_{Y, Y^{\prime}}\right) \cdot\left(\sum_{X, X^{\prime} \in \mathrm{Ob} \mathcal{C}} g_{X, X^{\prime}}\right)+\left(\sum_{Y, Y^{\prime} \in \mathrm{Ob} \mathcal{C}} h_{Y, Y^{\prime}}\right) \cdot\left(\sum_{X, X^{\prime} \in \mathrm{Ob} \mathcal{C}} f_{X, X^{\prime}}\right)
\end{aligned}
$$


- (Right distributive property)

$$
\begin{aligned}
\sum_{X, X^{\prime} \in \mathrm{Ob} \mathcal{C}} g_{X, X^{\prime}} & \left.+\sum_{X, X^{\prime} \in \mathrm{Ob} \mathcal{C}} f_{X, X^{\prime}}\right) \cdot \sum_{Y, Y^{\prime} \in \mathrm{Ob} \mathcal{C}} h_{Y, Y^{\prime}} \\
& =\left(\sum_{X, X^{\prime} \in \mathrm{Ob} \mathcal{C}}\left(g_{X, X^{\prime}}+f_{X, X^{\prime}}\right)\right) \cdot \sum_{Y, Y^{\prime} \in \mathrm{Ob} \mathcal{C}} h_{Y, Y^{\prime}} \\
& =\left(\sum_{X, X^{\prime}, Y, Y^{\prime} \in \mathrm{Ob} \mathcal{C}}\left(g_{X, X^{\prime}}+f_{X, X^{\prime}}\right) \circ h_{Y, Y^{\prime}}\right) \\
& =\left(\sum_{X, X^{\prime}, Y, Y^{\prime} \in \mathrm{Ob} \mathcal{C}} g_{X, X^{\prime}} \circ h_{Y, Y^{\prime}}+f_{X, X^{\prime}} \circ h_{Y, Y^{\prime}}\right) \\
& =\left(\sum_{X, X^{\prime} Y \in \mathrm{Ob} \mathcal{C}} g_{X, X^{\prime}} \circ h_{Y, X}+f_{X, X^{\prime}} \circ h_{Y, X}\right) \\
& =\left(\sum_{X, X^{\prime}, Y \in \mathrm{Ob} \mathcal{C}} g_{X, X^{\prime}} \circ h_{Y, X}\right)+\left(\sum_{X, X^{\prime}, Y \in \mathrm{Ob} \mathcal{C}} f_{X, X^{\prime}} \circ h_{Y, X}\right) \\
& =\left(\sum_{X, X^{\prime} \in \mathrm{Ob} \mathcal{C}} g_{X, X^{\prime}}\right) \cdot\left(\sum_{Y, Y^{\prime} \in \mathrm{Ob} \mathcal{C}} h_{Y, Y^{\prime}}\right)+\left(\sum_{X, X^{\prime} \in \mathrm{Ob} \mathcal{C}} f_{X, X^{\prime}}\right) \cdot\left(\sum_{Y, Y^{\prime} \in \mathrm{Ob} \mathcal{C}} h_{Y, Y^{\prime}}\right)
\end{aligned}
$$

It is straightforward to verify that $1_{R_{C}}$ is the sum of all elements in $I_{C}$.

\section{AJUR Volume 12 | Issue 4 | November 2015}




\section{EQUIVALENCES}

In this section, we prove an equivalence of categories between the category of rings and the category of small preadditive categories with one object starting with Proposition 6.1 and between the category of idempotented rings and the category of small preadditive categories with finitely many objects under Proposition 6.4.

Proposition 6.1 The category Ring is equivalent to the category PreaddCat 1.

Proof.

We define a functor $G$ : PreaddCat ${ }_{1} \rightarrow$ Ring as follows:

$$
\begin{gathered}
G(C)=R_{C} \in \text { Ob Ring for all } C \in \text { Ob PreaddCat }_{1}, \\
G(H)(r)=(H)(r) \in \operatorname{Mor} \mathcal{D}(*, *) \text { for all } r \in \operatorname{Mor}_{C}(*, *) \text { and } H \in \text { MorPreaddCat }_{1}(C, \mathcal{D}) .
\end{gathered}
$$

That is, $G$ maps a preadditive category to its corresponding ring. Let $H \in \operatorname{MorPreaddCat}_{1}(C, \mathcal{D})$, an additive functor between preadditive categories $C$ and $\mathcal{D}$ containing one object. By Lemma 5.2, any $H \in$ MorPreaddCat $t_{1}(C, D)$ is a ring homomorphism. So we define $G(H)$ to be this ring homomorphism. Then $G(H) \in \operatorname{Mor} R i n g(G(C), G(\mathcal{D}))=\operatorname{MorRing}\left(R_{C}, R \mathcal{D}\right)=\operatorname{Mor} R i n g(\operatorname{Mor} C(* *), \operatorname{Mor} \mathscr{D}(*, *))$. It is straightforward to verify that $G \circ F=\mathrm{id}$ Ring and that $G$ is a functor.

- (Preserves identity morphisms) Let $C \in$ Ob PreaddCat 1 . Then for all $r \in \operatorname{Mor} C(*, *)$, $G\left(\operatorname{id}_{C}\right)(r)=\operatorname{id}_{C}(r)=r$, and so $G\left(\operatorname{id}_{C}\right)$ is the identity ring homomorphism from $R_{C}$ to $R_{C}$.

- (Preserves composition) Let $C, \mathcal{D}, \mathcal{E} \in$ Ob PreaddCat ${ }_{1}$ with additive functors $H: C \rightarrow \mathcal{D}$ and $H^{\prime}: \mathcal{D} \rightarrow \mathcal{E}$. Then for all $r \in R, G\left(H^{\prime} \circ H\right)(r)=H^{\prime} \circ H(r)=G\left(H^{\prime}\right) \circ G(H)(r) \in \operatorname{Mor} \mathcal{E}(*, *)$. We claim that $G$ yields an equivalence of Ring and PreaddCat 1 .

- (Full) Let $R, S \in \mathrm{Ob}$ Ring and $h \in \operatorname{Mor} \operatorname{Ming}(R, S)$. Define categories $C_{R}, C_{S} \in$ Ob PreaddCat $_{1}$ where $\mathrm{Ob}_{R}=\{*\}, \operatorname{Mor}_{R}(*, *)=R$ and $\mathrm{Ob} C_{S}=\{*\}, \operatorname{Mor}_{C_{S}}(*, *)=S$. Next we construct a functor $H: C_{R} \rightarrow C_{S}$ that maps the singleton object $* \in \mathrm{Ob} C_{R}$ to $* \in \mathrm{Ob} C_{S}$. Then for any $r \in \operatorname{Mor}_{R}(*, *), H(r)=h(r) \in \operatorname{Mor}_{S}(*, *)$. Thus, $G(H)=h$.

- (Faithful) Let $H, J \in \operatorname{MorPreaddCat}_{1}(C, \mathcal{D})$, be such that $G(H)=G(J)$. Since $\mathrm{Ob} C$ and $\mathrm{Ob} \mathcal{D}$ consist of only one object *, $H$ and $J$ must map them to each other, so $H$ and $J$ are the same on objects. Furthermore we have that:

$$
\begin{gathered}
G(H)=G(J) \\
\Longrightarrow G(H)(r)=G(J)(r) \text { for all } r \in R \\
\Longrightarrow H(r)=J(r) \text { for all } r \in R \\
\Longrightarrow H=J
\end{gathered}
$$

- (Essentially surjective) For all $R \in \mathrm{Ob}$ Ring, we construct the category $C_{R} \in$ PreaddCat 1 where $\mathrm{Ob} C_{R}=\{*\}$ and $\operatorname{Mor}_{R}\left({ }^{*},{ }^{*}\right)=R$. So $G\left(C_{R}\right)=R$ and clearly $R \cong R$. 
The following proposition shows that the categories Ring and PreaddCat 1 are equivalent not only as 1-categories, but also as 2-categories.

Proposition 6.2 The 2-category Ring is equivalent to the 2-category PreaddCat ${ }_{1}$ as 2-categories.

Proof.

From Proposition 6.1, we defined the fully faithful and essentially surjective functor $G$ : PreaddCat $_{1}$ $\rightarrow$ Ring to show that Ring $\simeq$ PreaddCat ${ }_{1}$ as 1 -categories. To prove their equivalence as 2 -categories, we will extend this functor $G:$ PreaddCat $_{1} \rightarrow$ Ring to a 2 -functor by defining its behaviour on 2-morphisms. Let $C, \mathcal{D} \in \mathrm{Ob}_{\text {PreaddCat }}, H, J \in$ MorPreaddCat $_{1}(C, D)$. Then for any $\alpha: H \Rightarrow J$,

$$
G(\alpha): G(H) \Rightarrow G(J) \quad G(\alpha)=\alpha_{*} \in \mathrm{R} \mathcal{D}=\operatorname{Mor} \mathscr{D}(*, *) .
$$

The functor $G$ is an extension of the 1 -functor mentioned in Proposition 6.1 so it has been proven that it preserves identity 1-morphisms and the composition of 1-morphisms. We will verify that $G$ is a 2 -functor by the following:

- (Preserves vertical composition) For $C$ and $\mathcal{D}$ in Ob PreaddCat $1, H, J, K: C \rightarrow \mathcal{D}, \alpha: H \Rightarrow J$ and $\beta: J \Rightarrow K$, we have that $G\left(\beta \circ_{\nu} \alpha\right)=\left(\beta \circ_{\nu} \alpha\right)_{*}=\beta *_{*} \circ_{\nu} \alpha *=G(\beta) \circ_{\nu} G(\alpha): G(H) \Rightarrow G(K)$.

- (Preserves horizontal composition) For $\mathcal{C}, \mathcal{D}, \mathcal{E} \in$ Ob PreaddCat $_{1}, H, J: C \rightarrow \mathcal{D}, H^{\prime}, J^{\prime}: \mathcal{D} \rightarrow \mathcal{E}$, $\alpha: H \Rightarrow J$ and $\alpha^{\prime}: H^{\prime} \Rightarrow J^{\prime}$, we have that $G\left(\alpha^{\prime} \circ h \alpha\right)=\left(\alpha^{\prime} \circ h \alpha\right)_{*}=\alpha_{*}^{\prime} \circ_{\nu} G\left(J^{\prime}\right)\left(\alpha_{*}\right)=G\left(\alpha^{\prime}\right) \circ h G(\alpha)$ : $G\left(H^{\prime} \circ H\right) \Rightarrow G\left(J^{\prime} \circ J\right)$.

- (Preserves identity 2-morphisms) For $C \in \mathrm{Ob}_{\text {PreaddCat }}, \operatorname{id}_{C}: C \rightarrow C$ and $\operatorname{id}_{i_{C}}: \operatorname{id}_{C} \Rightarrow \operatorname{id}_{C}$, we have $G\left(\operatorname{id}_{i_{C}}\right)=\operatorname{id}_{\operatorname{id}_{C_{*}}}=\operatorname{id}_{*}: \operatorname{id}_{i C_{C_{R}}} \Rightarrow \operatorname{id}_{\operatorname{id}_{C_{R}}}$.

We claim that $G$ yields an equivalence of Ring and PreaddCat ${ }_{1}$ as 2-categories. We know that $G$ is essentially surjective on objects and fully faithful on 1-morphisms from the proof that Ring $\simeq$ PreaddCat 1 as 1-categories from Proposition 6.1, so we just need to show that $G$ is fully faithful on 2-morphisms.

- (Faithful on 2-morphisms) Let $C, \mathcal{D} \in \mathrm{Ob}$ PreaddCat $_{1}, H, J \in$ MorpreaddCat $_{1}(C, \mathcal{D})$ and $\alpha, \beta: H \Rightarrow J$. If we assume that $G(\alpha)=G(\beta)$, then we have that $\alpha_{*}=\beta_{*}$. This implies that $\alpha=\beta$ since $\mathrm{Ob} C$ contains only one object $*$ and both natural transformations assign the same morphism to this object.

- (Full on 2-morphisms) Let $R, S \in \mathrm{Ob} \operatorname{Ring}, f, h \in \operatorname{Mor}_{\text {Ring }}(R, S)$ and $\alpha: f \Rightarrow g$. We define objects $C_{R}$ and $C_{S} \in \mathrm{Ob}$ PreaddCat 1 where $\mathrm{Ob} C_{R}=\{*\}, \operatorname{Mor}_{C_{R}}(*, *)=R$ and $\mathrm{Ob} C_{S}=\{*\}$, $\operatorname{Mor} C_{S}(*, *)$. Thus $\alpha$ is a morphism in $\operatorname{Mor} C_{S}(*, *)$. Since $G$ is fully faithful on 1-morphisms, there exist functors $F, H: C_{R} \rightarrow C_{S}$ such that $G(F)=f$ and $G(H)=h$. Then let $\eta: F \Rightarrow H$ be the natural transformation that associates to $* \in \mathrm{Ob} C_{R}$ the morphism $\alpha$ in $\operatorname{Mor}_{C_{S}}(* * *)$. This then gives us that $G(\eta)=\eta_{*}=\alpha: f \Rightarrow h$. 
The following proposition explains the ring-theoretic analogue of adjoint functors, under the correspondences given above.

Proposition 6.3 For $C, \mathcal{D} \in \mathrm{Ob}_{\operatorname{PreaddCat}} H \in \operatorname{MorPreaddCat}_{1}(\mathcal{C}, \mathcal{D})$ and $J \in \operatorname{MorPreaddCat}_{1}(\mathcal{D}, \mathcal{C})$, let $G(C)=R, G(D)=S, G(H)=h \in \operatorname{MorRing}(R, S), G(J)=j \in \operatorname{MorRing}(S, R)$. Then H is left adjoint to $J$ if and only if there exist 2-morphisms $\eta: \mathrm{id}_{R} \Rightarrow j \circ h$ and $\epsilon: h \circ j \Rightarrow \mathrm{id}_{S}$ in Ring such that the maps $\phi: r \mapsto \epsilon h(r)$ and $\psi: s \mapsto j(s) \eta$ are mutually inverse bijections of sets.

Proof. $\Longrightarrow$ Assume that $H \dashv J$. By the definition of adjointness, there exist natural transformations $\eta: \operatorname{id}_{C} \Rightarrow J \circ H$ and $\epsilon: H \circ J \Rightarrow \operatorname{id} \mathcal{D}$ such that $\epsilon_{H(*)} H\left(\eta_{*}\right)=\operatorname{id}_{\left.J_{(*}\right)}$ and $J\left(\epsilon_{*}\right) \eta_{*}=\operatorname{id}_{H(*)}$. Under the correspondences given in Proposition 6.2, the natural transformations $\eta$ and $\epsilon$ correspond to 2-morphisms in Ring such that the following equations hold:

$$
\begin{aligned}
\eta r=j h(r) \eta, & \text { for all } r \in R, \\
\epsilon h j(s)=s \epsilon, & \text { for all } s \in S .
\end{aligned}
$$

Furthermore, since $H \dashv J$, we have that $\epsilon h(\eta)=1_{S}$ and $j(\epsilon) \eta=1_{R}$ in Ring. Then, for any $r \in R$, we have

$$
\psi(\phi(r))=\psi(\epsilon h(r))=j(\epsilon h(r)) \eta=j(\epsilon)(j h(r)) \eta=j(\epsilon) \eta r=r,
$$

and for any $s \in S$,

$$
\phi(\psi(s))=\phi(j(s) \eta)=\epsilon h(j(s) \eta)=\epsilon(h j(s)) h(\eta)=s \epsilon h(\eta)=s .
$$

So $\phi$ and $\psi$ are mutually inverse bijections of sets.

$\Longleftarrow$ Assume that $\eta: \operatorname{id}_{R} \Rightarrow j \circ h$ and $\epsilon: h \circ j \Rightarrow$ id $s$ are 2-morphisms in Ring and that $\phi: r \mapsto \epsilon h(r)$ and $\psi: s \mapsto j(s) \eta$ are mutually inverse bijections of sets. By the definition of a 2-morphism in Ring, for any $r \in R$, we have that $\eta r=j h(r) \eta$. Likewise, for any $s \in S, \epsilon h j(s)=s \epsilon$. Since $\psi$ is the left-inverse of $\phi, \psi(\phi(r))=\operatorname{id}_{R}(r)$ for all $r \in R$. Taking $r=1_{R}$, we get that

$$
\psi\left(\phi\left(1_{R}\right)\right)=\operatorname{id}_{R}\left(1_{R}\right) \Longrightarrow j\left(\epsilon h\left(1_{R}\right)\right) \eta=1_{R} \Longrightarrow j(\epsilon) j \circ h\left(1_{R}\right) \eta=1_{R} \Longrightarrow j(\epsilon) \eta=1_{R} .
$$

Similarly, since $\phi$ is the left-inverse of $\psi, \phi(\psi(s))=\operatorname{id} s(s)$ for all $s \in S$. Taking $s=1 s$, we get that

$$
\phi\left(\psi\left(1_{S}\right)\right)=\operatorname{id}_{S}\left(1_{S}\right) \Longrightarrow \epsilon h\left(j\left(1_{S}\right) \eta\right)=1_{S} \Longrightarrow \epsilon h \circ j\left(1_{S}\right) h(\eta)=1_{S} \Longrightarrow \epsilon h(\eta)=1_{S} .
$$

We can interpret $R$ and $S$ respectively as categories $C$ and $\mathcal{D}$ with a formal object whose morphisms are the rings themselves. Then the ring homomorphisms $j$ and $h$ correspond to functors $J: C \rightarrow D$ and $H: D \rightarrow C$. Similarly, the 2-morphisms $\epsilon$ and $\eta$ in Ring coincide with natural transformations $\epsilon: H \circ J \Rightarrow \operatorname{id} \mathcal{D}$ and $\eta: \operatorname{id}_{C} \rightarrow J \circ H$ in PreaddCat 1 . Thus the equations $j(\epsilon) \eta=1_{R}$ and $\epsilon h(\eta)=1_{S} \mathrm{im}$ plies that $\epsilon_{H(*)} H\left(\eta_{*}\right)=\operatorname{id} J(*)$ and $J\left(\epsilon_{*}\right) \eta_{*}=\operatorname{id}_{H(*)}$ and so $H$ is left adjoint to $J$.

Proposition 6.4 The category Ring $\perp$ is equivalent to the category PreaddCatFin.

Proof.

We define an additive functor $G$ : PreaddCat $F i n \rightarrow$ Ring $_{\perp}$ as follows:

$$
\begin{gathered}
G(\mathcal{C})=\left(R_{\mathcal{C}}, I_{\mathcal{C}}\right) \in \mathrm{Ob} R i n g_{\perp} \quad \text { for all } \mathcal{C} \in \text { Ob PreaddCat }_{\mathrm{Fin}}, \\
G(H)\left(\sum_{X, Y \in \mathrm{Ob} \mathcal{C}} f_{X, Y}\right)=\left(\sum_{X, Y \in \mathrm{Ob} \mathcal{C}} H\left(f_{X, Y}\right)\right) \text { for all } H \in \operatorname{Mor}_{\text {PreaddCat }_{F i n}}(C, D),
\end{gathered}
$$

Note that for any $C, \mathcal{D} \in \mathrm{Ob}$ PreaddCat Fin, we have the multisets $I_{C}=O b C$ and $I_{\mathcal{D}}=\mathrm{Ob} \mathcal{D}$. Then for $H: C \rightarrow \mathcal{D}$ and $X \in O b C, G(H)\left(\operatorname{id}_{X}\right)=H\left(\operatorname{id}_{X}\right)=\operatorname{id}_{H(X)} \in I_{\mathcal{D}}$. Thus the distinguished sets $I_{C}$ and $I_{\mathcal{D}}$ are respected by the morphism $G(H) \in \operatorname{Mor}_{\text {PreaddCatFin }}\left(\left(R_{C}, I_{C}\right),\left(R_{\mathcal{D}}, I_{\mathcal{D}}\right)\right)$. Furthermore, it is 
important to point out that for $\sum_{X, Y \in O b C} f_{X, Y} \in R_{C}$, the element $G(H)\left(\sum_{X, Y \in O b C} f_{X, Y}\right)$ may not be a direct sum since it is possible that $G$ is not injective on objects. With that being said, we proceed to verify that $G$ is a functor below.

- (Preserves identity morphism) For any $C \in$ Ob PreaddCatFin,

$G\left(\operatorname{id}_{\mathcal{C}}\right)\left(\sum_{X, Y \in \mathrm{Ob} \mathcal{C}} f_{X, Y}\right)=\left(\sum_{X, Y \in \mathrm{Ob} \mathcal{C}} \operatorname{id}_{\mathcal{C}}\left(f_{X, Y}\right)\right)=\left(\sum_{X, Y \in \mathrm{Ob} \mathcal{C}} f_{X, Y}\right)=\operatorname{id}_{G(\mathcal{C})}\left(\sum_{X, Y \in \mathrm{Ob} \mathcal{C}} f_{X, Y}\right)$.

- (Preserves Composition) Let $C, \mathcal{D}, \mathcal{E} \in$ Ob PreaddCatFin with morphisms $H: C \rightarrow \mathcal{D}$ and $H^{\prime}: \mathcal{D} \rightarrow \mathcal{E}$. For all $\sum_{X, Y \in O b C} f X, Y$,

$G\left(H^{\prime} \circ H\right)\left(\sum_{X, Y \in \mathrm{Ob} \mathcal{C}} f_{X, Y}\right)=\sum_{X, Y \in \mathrm{Ob} \mathcal{C}} H^{\prime} \circ H\left(f_{X, Y}\right)=G\left(H^{\prime}\right) \circ G(H)\left(\sum_{X, Y \in \mathrm{Ob} \mathcal{C}} f_{X, Y}\right)$.

We claim that $G$ yields an equivalence of Ring $\perp$ and PreaddCat $F i n$.

- (Full) Let $h \in \operatorname{Mor}_{R_{i n g}}(G(C), G(\mathcal{D}))=\operatorname{Mor}_{R_{i n g}}\left(\left(R_{C}, I_{C}\right),\left(R_{D}, I_{D}\right)\right)$. Then we define a functor $H \in$ MorPreaddCat $_{\mathrm{Fin}}(\mathcal{C}, \mathcal{D})$ as follows:

$$
\begin{gathered}
H(X)=\operatorname{Dom}\left(h\left(\operatorname{id}_{X}\right)\right) \quad \text { for all } X \in \operatorname{Ob} C \\
\mathrm{H}\left(f_{X, Y}\right)=h\left(f_{X, Y}\right) \quad \text { for all } f_{X, Y} \in \operatorname{Mor}_{C}(X, Y) \text { and } X, Y \in \operatorname{Ob} C .
\end{gathered}
$$

Then $H\left(f_{X, Y}\right)$ is a morphism from $H(X)$ to $H(Y)$ since $H\left(f_{X, Y}\right)=H\left(\operatorname{id}_{Y} f_{X, Y} \operatorname{id} X\right)=H\left(\operatorname{id}_{Y}\right) H\left(f_{X, Y}\right)$ $H\left(\operatorname{id}_{X}\right)=\operatorname{id}_{H(Y)} H\left(f_{X, Y}\right) \operatorname{id}_{H(X)}$. So for all $\left(\sum_{X, Y \in O b C} f_{X, Y}\right) \in\left(R_{C}, I_{C}\right)$, we have that:

$$
\begin{aligned}
G(H)\left(\sum_{X, Y \in \mathrm{Ob} \mathcal{C}} f_{X, Y}\right) & =\left(\sum_{X, Y \in \mathrm{Ob} \mathcal{C}} H\left(f_{X, Y}\right)\right) \\
& =\left(\sum_{X, Y \in \mathrm{Ob} \mathcal{C}} h\left(f_{X, Y}\right)\right) \\
& =h\left(\sum_{X, Y \in \mathrm{Ob} \mathcal{C}} f_{X, Y}\right) \\
& \Longrightarrow G(H)=h
\end{aligned}
$$

- (Faithful) Assume that $G(H)=G(J), H, J \in \operatorname{MorPreaddCat}_{\text {Fin }}(C, \mathcal{D})$. Since $G(H)$ and $G(J)$ are the same ring homomorphism, they are the same map on the idempotents. Thus for all $X \in O b C$, we have that $G(H)\left(\mathrm{id}_{X}\right)=G(J)\left(\mathrm{id}_{X}\right)$ and that $H\left(\mathrm{id}_{X}\right)=J\left(\mathrm{id}_{X}\right)$. This implies that $\operatorname{id}_{H(X)}=\operatorname{id}_{J(X)}$ and so $H(X)=J(X)$, so $H$ and $J$ must be the same map on objects. More generally, for all $f_{X, Y} \in R_{C}$,

$$
\begin{gathered}
G(H)\left(f_{X, Y}\right)=G(J)\left(f_{X, Y}\right) \\
\Longrightarrow\left(\sum_{X, Y \in \mathrm{Ob} \mathcal{C}} H\left(f_{X, Y}\right)\right)=\left(\sum_{X, Y \in \mathrm{Ob} \mathcal{C}} J\left(f_{X, Y}\right)\right) \\
\Longrightarrow H\left(f_{X, Y}\right)=J\left(f_{X, Y}\right) \quad \text { for all } X, Y \in \mathrm{Ob} \mathcal{C}, f_{X, Y} \in \operatorname{Mor}_{\mathcal{C}}(X, Y) \\
\Longrightarrow H=J
\end{gathered}
$$

\section{AJUR Volume 12 | Issue 4 | November 2015}


- (Essentially surjective) For all $(R, I) \in \mathrm{Ob}$ Ring $\perp$ where $I=\left\{e_{1}, e_{2}, \cdots, e_{n}\right\}$ we are able to construct the preadditive category $C_{(R, I)}$ where $\operatorname{Ob} C_{(R, I)}=I$ and $\operatorname{Mor}_{(R, I)}\left(e_{i}, e_{j}\right)=e_{j} R e_{i}$ for all $e_{i}, e_{j} \in \mathrm{Ob} C_{(R, I)}$. We claim that $\mathrm{G}\left(C_{(R, I)} \cong\left(M_{n}(R), I_{\left.M_{n}(R)\right)}\right.\right.$. Consider the function

$$
\phi:\left(M_{n}(R), I_{M_{n}(R)}\right) \rightarrow G\left(\mathcal{C}_{(R, I)}\right), \quad \phi\left(\left(e_{j} r e_{i}\right)\right)=\left(\sum_{i, j=1}^{n} e_{j} r e_{i}\right),
$$

where $\left(e_{j} r e_{i}\right)$ is the $n \times n$ matrix whose $(i, j)$ entry is $e_{j} r e_{i}$. Then the proof of most of the ring isomorphism axioms are essentially the same as the ones from Example 2.6. Having just proved that $G\left(C_{(R, I)}\right) \cong\left(M_{n}(R), I_{M_{n}(R)}\right)$, Proposition 2.6 gives us that $\left(M_{n}(R), I_{M_{n}(R)}\right) \cong(R, I)$, and so $G\left(C_{(R, I)} \cong(R, I)\right.$.

The following proposition shows that equivalence of 1-categories between Ring $\perp$ and PreaddCat Fin can be extended to 2-categories.

Proposition 6.5 The 2-category Ring $\perp$ is equivalent to the 2-category PreaddCatFin as 2-categories.

Proof.

In Proposition 6.4, we defined the 1 -functor $G$ to prove that Ring $\perp$ and PreaddCat Fin $_{\text {in }}$ are equivalent as 1-categories. We can extend our 1-functor $G$ into a 2 -functor by defining its behaviour on 2-morphisms with the following. Let $C, \mathcal{D} \in$ Ob PreaddCatFin, $H, J: C \rightarrow \mathcal{D}$. Then for any $\alpha: H \Rightarrow J$, the 2-morphism $G(\alpha): G(H) \Rightarrow G(J)$ is the mapping from $I_{C}$ to $R_{\mathcal{D}}$ such that for any $X \in \mathrm{Ob} C$,

$$
G(\alpha)\left(\operatorname{id}_{X}\right)=\alpha_{X} \in G(\mathcal{D})=R \mathscr{D} .
$$

Likewise, $G$ is also an extension of the 1-functor in Proposition 6.4 where it was proven that $G$ preserves identity 1-morphisms and the composition of 1-morphisms. Thus we check that it is indeed a 2 -functor with the following:

- (Preserves vertical composition) Let $C, \mathcal{D} \in$ Ob PreaddCatFin, $H, J, K: C \rightarrow \mathcal{D}, \alpha: H \Rightarrow J$ and $\beta: J \Rightarrow K$. Then for any $X \in \mathrm{Ob} C, G\left(\beta \circ_{\nu} \alpha\right)\left(\mathrm{id}_{X}\right)=\left(\beta \circ_{\nu} \alpha\right)(\mathrm{X})=\beta_{X} \circ \alpha_{X}=G(\beta)\left(\mathrm{id}_{X}\right) \circ_{\nu} G(\alpha)\left(\mathrm{id}_{X}\right)$.

- (Preserves horizontal composition) Let $C, \mathcal{D}, \mathcal{E} \in$ Ob PreaddCatFin, $H, J: C \rightarrow \mathcal{D}, H^{\prime}, J^{\prime}$ : $\mathcal{D} \rightarrow \mathcal{E}, \alpha: H \Rightarrow J$ and $\alpha^{\prime}: H^{\prime} \Rightarrow J^{\prime}$. Then for any $X \in \mathrm{Ob} C, G\left(\alpha^{\prime} \circ h \alpha\right)\left(\operatorname{id}_{X}\right)=\left(\alpha^{\prime} \circ h \alpha\right)(\operatorname{id} X)=$ $\alpha^{\prime} G(J)(X) \circ G(J)\left(\alpha_{x}\right)=G\left(\alpha^{\prime}\right)\left(\operatorname{id}_{X}\right) \circ h G(\alpha)\left(\operatorname{id}_{X}\right)$.

- (Preserves identity 2-morphisms) Let $C \in \mathrm{Ob}_{\text {PreaddCatFin, }} \operatorname{id}_{C}: C \rightarrow C$ and $\operatorname{idid}_{C}: \operatorname{id}_{C} \Rightarrow \operatorname{id}_{C}$. Then for any $X \in \mathrm{Ob} C, G\left(\operatorname{idid}_{C}\right)\left(\operatorname{id}_{X}\right)=\operatorname{idid}_{C}=\operatorname{id} X$.

We claim that $G$ yields an equivalence of 2 -categories between the Ring $\perp$ and PreaddCatFin.

- (Faithful on 2-morphisms) Let $C, \mathcal{D} \in$ Ob PreaddCatFin, $h, j \in$ MorRing $_{\perp}(G(C), G(\mathcal{D})$ ) and $\alpha, \beta: h \Rightarrow j$. Assume that $G(\alpha)=G(\beta)$. Then for any $X \in \mathrm{Ob} C$, we have that

$$
G(\alpha)=G(\beta) \Longrightarrow G(\alpha)\left(\mathrm{id}_{X}\right)=G(\beta)\left(\mathrm{id}_{X}\right) \Longrightarrow \alpha X=\beta X \Longrightarrow \alpha=\beta \text {. }
$$

- (Full on 2-morphisms) Let $C, \mathcal{D} \in$ Ob PreaddCatFin, $h, j: G(C) \rightarrow G(\mathcal{D})$ and $\alpha: h \Rightarrow j$. The 2-morphism $\alpha$ has the property that for any $\operatorname{id}_{X}$ and $\operatorname{id}_{Y}$ in $I_{C}, \alpha\left(\operatorname{id}_{X}\right)$ is an element in $\mathrm{R}_{\mathcal{D}}$ such that

$$
j\left(\operatorname{id}_{Y}\left(\sum_{X, Y \in \mathrm{Ob}_{\mathcal{C}}} f_{X, Y}\right) \mathrm{id}_{X}\right) \alpha\left(\operatorname{id}_{X}\right)=\alpha\left(\operatorname{id}_{Y}\right) h\left(\operatorname{id}_{Y}\left(\sum_{X, Y \in \mathrm{Ob} \mathcal{C}} f_{X, Y}\right) \operatorname{id}_{X}\right)
$$

for any $\sum_{X, Y \in O b C} f_{X, Y} \in R_{C}$. The functor $G$ is surjective on 1-morphisms, so let $H, J: C \rightarrow \mathcal{D}$ such that $G(H)=h$ and $G(J)=j$. We construct a natural transformation $\beta: H \Rightarrow J$ as so: for any $X \in \operatorname{Ob} C, \beta_{X}=\alpha\left(\operatorname{id}_{X}\right)$. Any $f \in \operatorname{Mor}_{C}(X, Y)$ is an element in $R_{C}$, so we have that $\beta_{X} H(f)=J(f) \beta_{Y}$, thus proving naturality of $\beta$. Then for all $\operatorname{id}_{X} \in R_{C}, G(\beta)\left(\operatorname{id}_{X}\right)=\beta_{X}=\alpha\left(\operatorname{id}_{X}\right)$ and so $G(\beta)=\alpha$. 


\section{ACKNOWLEDGMENTS}

This work was completed as part of a Co-op Work-Study Program at the University of Ottawa. The author thanks the University of Ottawa and the Department of Mathematics and Statistics for its support. He also thanks Alistair Savage for guidance throughout the project.

\section{REFERENCES}

[1] Wiles, A. (1995) Modular elliptic curves and Fermat's last theorem. Ann. of Math. (2), 141(3):443-551.

[2] Noether, E. (1921) Idealtheorie in Ringbereichen. Math. Ann, 83(1-2):24-66.

[3] Eilenberg, S., and MacLane, S. (1945) General theory of natural equivalences. Trans. Amer. Math. Soc, 58:231-294.

[4] Grothendieck, A., and Dieudonné, J. A. (1961) Eléments de géométrie algébrique. I, volume 5 of Grundlehren der Mathematischen Wissenschaften. Springer-Verlag, Berlin.

[5] Lawvere, F. W. (1963) Functorial semantics of algebraic theories. Trans. Amer. Math. Soc., 50:869-872.

[6] Chuang, J., and Rouquier, R. (2008) Derived equivalences for symmetric groups and $\mathfrak{S l}_{2} 2$-categorication. Ann. of Math. (2), 167(1):245-298.

[7] Khovanov, M., and Lauda. A. D. (2009) A diagrammatic approach to categorication of quantum groups. I. Represent. Theory, 13:309-347.

[8] Khovanov, M., and Lauda. A. D. (2010) A categorication of quantum sl(n). Quantum Topol., 1(1):1-92.

[9] Khovanov, M., and Lauda. A. D. (2011) A diagrammatic approach to categorication of quantum groups II. Trans. Amer. Math. Soc., 363(5):2685-2700.

[10] Lauda, A. D. (2010) A categorication of quantum sl(2). Adv. Math., 225(6):3327-3424.

[11] Lusztig, G. (1990) Canonical bases arising from quantized enveloping algebras. J. Amer. Math. Soc., 3(2):447-498.

[12] Mac Lane, S. (1998) Categories for the working mathematician, volume 5 of Graduate Texts in Mathematics. Springer-Verlag, New York, second edition, p. 93.

[13] Kelly, G. M. (2005) Basic concepts of enriched category theory. Repr. Theory Appl. Categ., (10): vi+137. Reprint of the 1982 original [Cambridge Univ. Press, Cambridge], p. 24.

\section{ABOUT THE STUDENT AUTHOR}

Edward Poon is a Toronto native who is currently going into his fourth year of the Honours B.Sc. in Mathematics. His research interests include category theory, rings and algebras.

\section{PRESS SUMMARY}

Category theory is a field of mathematics that can be used to study various algebraic structures including rings, which provide a generalization of arithmetic. In particular, a ring can be interpreted as a category and vice-versa. This paper delves into the relationship between categories and rings and some correspondences between the two fields with a view towards applications in the field of categorication. 


\title{
Effect of Manual Ivy Removal on Seedling Recruitment in Forest Park, Portland, OR
}

\author{
Katelin D. Stanley \& Dr. David W. Taylor \\ Department of Biology, University of Portland, Oregon \\ Student: kstanley@bio.fsu.edu,Mentor:taylorda@up.edu
}

\begin{abstract}
English and Irish ivy (Hedera helix and H. hibernica) are invasive lianas which have become especially intrusive in the Pacific Northwest, as evidenced by their invasion of many areas in Forest Park, Portland, OR. The most common strategy for ivy control is currently manual removal, though the potential consequences of this method have not been well studied. The purpose of this study was to evaluate the effectiveness of manual ivy removal with respect to its influence on native plant diversity, abundance, species richness, and evenness by comparing these parameters in $1-\mathrm{m}^{2}$ plots manually cleared of ivy to paired control plots. Prior to plot establishment, evidence of deer herbivory of ivy was observed at this site. Four weeks after manual removal, treated plots were less diverse and hosted a lower abundance of native plants than control plots. Ten weeks after ivy removal, treated and control plots were equally diverse, and treated plots showed greater abundance of plant cover than controls. This trend persisted at twenty-six weeks after treatment. Treated and control plots were not significantly different in species richness or evenness at any time. These results suggest that manual ivy removal temporarily disturbs native plant life, but the negative effects are overcome as quickly as ten weeks after treatment, at which time native plants are more successful. Manual removal as a method to control ivy in this region appears effective within one growing season
\end{abstract}

\section{KEYWORDS}

English Ivy, Irish Ivy, Invasive Species, Pacific Northwest, Forest Park, Hedera helix, Hedera hibernica

\section{INTRODUCTION}

English ivy (Hedera helix) and Irish ivy (Hedera hibernica), henceforth collectively denoted "ivy," are perennial lianas native to Europe which have escaped cultivation and become invasive in North America, especially the Pacific Northwest (PNW). ${ }^{1}$ Washington and Oregon have listed ivy as a noxious weed, and Oregon has even placed it under quarantine. ${ }^{2}$ Though ivy can reproduce sexually, in which its seeds are dispersed by birds, the success of ivy as an invasive species is more commonly attributed to its vegetative reproduction. ${ }^{3}$ Ivy demonstrates rapid vegetative growth and has been reported to ascend trees at a rate of up to $10 \mathrm{~m}$ per year using adventitious roots, resulting in both dominance of the understory and influence on forest canopies. ${ }^{4,5}$ Likely due to its evergreen nature and tolerance of heavy canopy cover, direct sunlight, and a wide range of soil pHs, ivy outcompetes native plant communities in forested areas. ${ }^{6,7}$ Ivy has been known to further disrupt forests by reducing root and leaf development of trees, ${ }^{8,9,10}$ predisposing host trees to weather-related damage, ${ }^{11}$ and preventing understory regeneration and growth by formation of near monotypic ivy mats. ${ }^{10,12}$ Ivy dominance has been correlated with alterations in soil nutrient contents, ${ }^{13}$ and these changes may have lasting impacts on native plant and animal communities. Furthermore, loss of native species diversity and abundance has the potential to reduce the functioning of the ecosystem. ${ }^{14}$

The PNW is a unique and sensitive region home to several endemic species such as the bigleaf maple, vine maple, and inside-out flower. The former two species may suffer from ivy's climbing ability, and each may experience displacement and increased competition from ivy groundcover. The hilly, suburban regions of Portland, Oregon are overrun by ivy in many places, and ivy has encroached on the city's largest urban forest reserve, Forest Park, where it forms dense mats and 
climbs native trees in disturbed areas. Because ivy invasion poses a direct threat to this region already experiencing anthropogenic habitat loss, an examination of ivy control methods is necessary to determine whether management efforts are being productively applied in this area.

Several studies examining possible solutions to the problem of invasive ivy have been conducted in recent years, with several methods of removal showing limited success. Control of ivy using herbicides has not proven effective due to the plant's thick cuticle ${ }^{15}$ though some exceptions have been indicated. ${ }^{16,17}$ Repeated burning and goat browsing have shown success in reducing ivy cover, but the efficacy of these methods is diminished by their significant time and resource requirements. ${ }^{10,18}$ To date, the favored ivy control strategy is manual removal - pulling the vines from the ground and vertical surfaces. ${ }^{19,20,21}$

While manual removal avoids many disadvantages of herbicidal and other treatments, this method, too, may have significant negative effects due to its disturbance of the soil and neighboring plant life. ${ }^{19}$ These effects have been considered minor relative to the consequences of ivy invasion, but few studies have investigated this assumption. The purpose of this study is to evaluate the effectiveness of manual ivy removal in terms of its impact on native plant diversity, abundance, species richness, and evenness. Because removing ivy has the potential to increase light and nutrient availability for native plants, it is hypothesized that greater native plant diversity and abundance will be observed in plots cleared of ivy over control plots.

\section{MATERIALS AND METHODS}

Ten pairs of 1- $\mathrm{m}^{2}$ ivy-covered (99-100\% cover) plots were marked with wooden stakes in northeast Forest Park (45.5924 N, -122.785 W). This location was chosen for its dense understory ivy growth, relatively low degree of slope, and ease of access, with plots sheltered from view yet still within 20 meters of a well-maintained trail. For each pair of plots, one was randomly designated as the treated plot and the other the control. Habitat characteristics such as nearby nursery logs or trees, overhanging branches, presence of surrounding plant species, and unique soil conditions were noted. Percent cover of all plant species was recorded using a 1- $\mathrm{m}^{2}$ PVC quadrat divided into $10 \times 10-\mathrm{cm}$ squares, and a photograph was taken for both control and treated plots upon establishment. Due to their morphological and niche similarities, the two species of ivy, if they were both present, were not differentiated. Plots were established over the course of approximately three weeks in February 2014.

All treated plots were manually cleared of ivy by hand-pulling and use of clippers on February 15th. As woody lateral stems often formed extensive under- and above-ground lattices, the entire underlying ivy network in each plot was removed. Care was taken not to tread in either plot during the removal process. Some native individuals remained after ivy removal, but most were unavoidably uprooted during the process. This effect did not appear dramatic, as the pre-treated average native plant cover for all plots excluding moss was only 10.4. Example photographs for control and treated plots before and after ivy removal are shown in Figure 1.
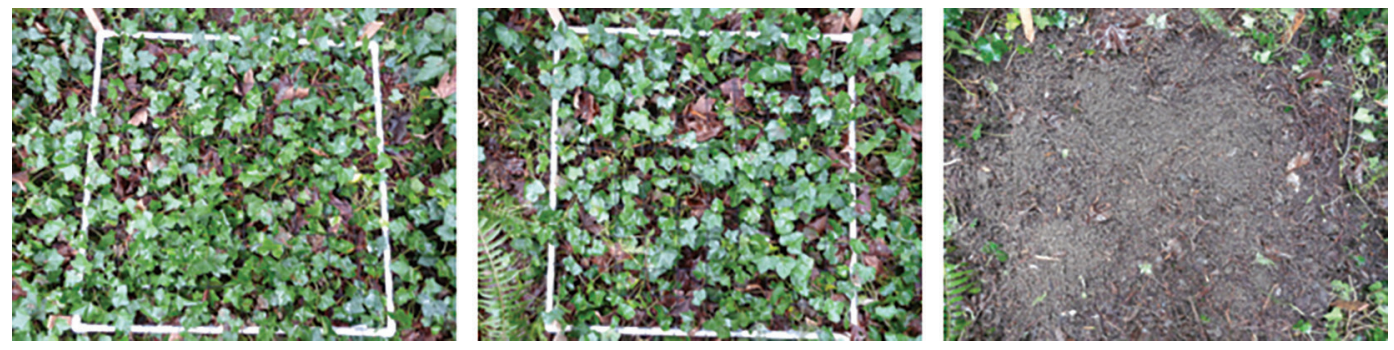

Figure 1. Example control plot (left) and treated plot photographs before (center) and after (right) manual ivy removal. Typical soil disturbance and incidental removal of detritus sustained during the clearing process is represented in the in the cleared treated plot (right). 
Sites were surveyed for vegetation three times after ivy removal: after four weeks (late Marchearly April), after ten weeks (early May), and after twenty-six weeks (mid-September). For each survey, the same quadrat used for plot establishment was placed with the corners at each stake. Percent cover of all plant species in the quadrat was recorded as the number of squares from which the species was observed to be growing. Any ivy root matter which had been overlooked during clearing (an average of 3.1 percent cover per plot) and new ivy growth were included in this survey. Due to the dense ivy mat and, in some plots, extensive growth of Hydrophyllum tenuipes (Pacific waterleaf), and manual manipulation of vegetation was necessary to ensure accurate records of plant presence. Control and treated plots were photographed after each survey. Plants were identified with help from a field guide ${ }^{22}$ and confirmed via taxonomic literature. ${ }^{23}$

Shannon-Weaver diversity indices were calculated based on percent cover values for each treated and control plot. Abundance of native plants was determined using the sum of percent cover values for all plant species in treated and control plots. Species richness and species evenness of all plots were also calculated. Percent cover of ivy was excluded from all calculations. Each variable was tested for significance using two-tailed t-tests. Euclidean distances were used to compare the statistical similarities of plot pairs after each survey; high values indicated high dissimilarity and low values indicated greater similarity between treated and control plots.

\section{RESULTS}

Regrowth of ivy into treated plots

By ten weeks after ivy removal, all but one treated plot contained at least one percent cover of ivy. Twenty-six weeks after treatment, ivy was observed in all treated plots, achieving an average of 12 percent cover.

\section{Native Plant Growth and Emergent Species}

Representative photographs of a treated plot at each of the survey dates are shown in Figure 2. In March/April, four weeks after ivy removal, seedlings were emerging in both control and treated plots, with a total of 13 species observed. After ten weeks, in May, native plant growth was abundant and six additional species were identified. In September, which was after a rather warm and dry summer, little plant growth was observed, though two additional species were identified. All 21 identifiable plant species that emerged during the survey period, with the exception of ivy, were Pacific Northwest natives. Though they could not be identified due to their minimal growth, several other morphologically distinct seedlings were included in analysis as unknowns. 

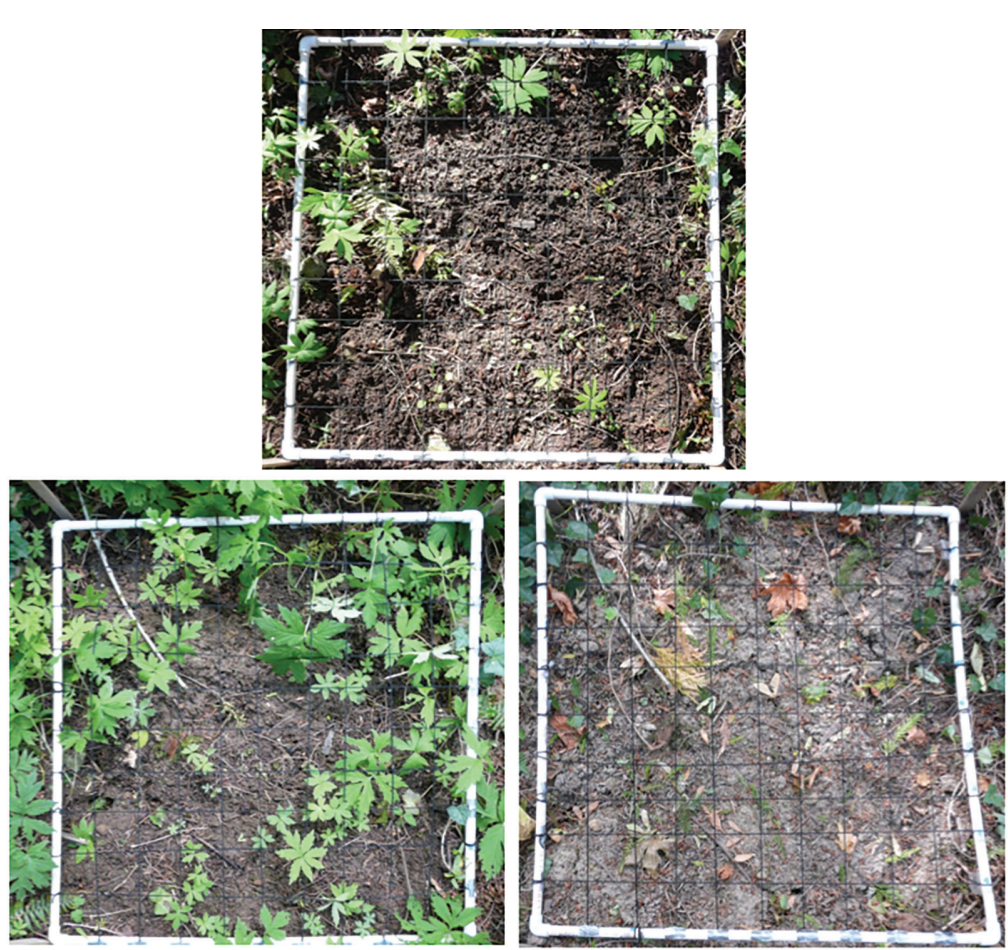

Figure 2. Example treated plot photographs after four weeks (top), after ten weeks (bottom left), and after twenty-six weeks (bottom right). This plot is representative of the average amount of growth observed in treated plots, though some plots showed much less cover and others showed much more. Individuals only observed as pairs of cotyledons, which proved difficult to identify, can be seen in each plot (see discussion). 


\begin{tabular}{|c|c|}
\hline Scientific Name & Common Name \\
\hline Acer circinatum & Vine maple \\
\hline Acer macrophyllum & Bigleaf maple \\
\hline Corylus spp.* & Hazelnut \\
\hline Hedera spp. & English or Irish ivy \\
\hline Hydrophyllum tenuipes & Pacific waterleaf \\
\hline Berberis nervosa & Dull Oregon grape \\
\hline Maianthemum racemosum ${ }^{*}$ & False Solomon's seal \\
\hline Oemleria cerasiformis & Indian plum \\
\hline Osmorhiza spp. & Sweet cicely \\
\hline Polypodium glycyrrhiza & Licorice fern \\
\hline Prosartes smithii & Hooker's fairy bells \\
\hline Rubus parviflorus & Thimbleberry \\
\hline Rubus spectabilis & Salmonberry \\
\hline Rubus ursinus & Trailing blackberry \\
\hline Smilacina racemosa & False Solomon's seal \\
\hline Trillium ovatum & Pacific trillium \\
\hline Unknown & Moss (unidentified) \\
\hline Unknown & Other bryophyte (unidentified; possible liverwort) \\
\hline Unknown, possibly Athyrium filix-femina & Fern (unidentified; possibly Common lady fern) \\
\hline Vancouveria hexandra & White inside-out flower \\
\hline Viola glabella & Stream violet \\
\hline
\end{tabular}

Table 1. List of identified species found in treated and control plots. Species in bold indicate those which were observed in early May, but not during late March/early April. An asterisk $\left({ }^{*}\right)$ denotes species observed only in September.

Effect of ivy removal on native plant diversity, abundance, species richness, and evenness

Four weeks after ivy removal, treated plots were less diverse (mean \pm SEM for Shannon $=1.15$ \pm 0.08 ) and less covered by native species (mean \pm SEM for total native cover $127 \pm 17$ ) than the control plots (Shannon $=1.35 \pm 0.06$, total native cover $159 \pm 15$ ) (Figure 3). Species richness and evenness were comparable between treated and control plots (mean \pm SEM for treated plots $=5.7 \pm$ 0.6 , control plots $=6.5 \pm 0.8$ ), and this trend persisted through subsequent surveys. Ten weeks after ivy removal, treated plots were as diverse (mean \pm SEM for Shannon $=1.08 \pm 0.08)$ as control plots (mean $\pm \mathrm{SEM}=1.03 \pm 0.08$ ) and covered by a higher abundance of native species (mean \pm SEM for total native cover of treated plots $=137 \pm 9$ and for control plots $119 \pm 13$ ). This trend lasted until the 26th week after ivy removal (Figure 3, Table 2). 


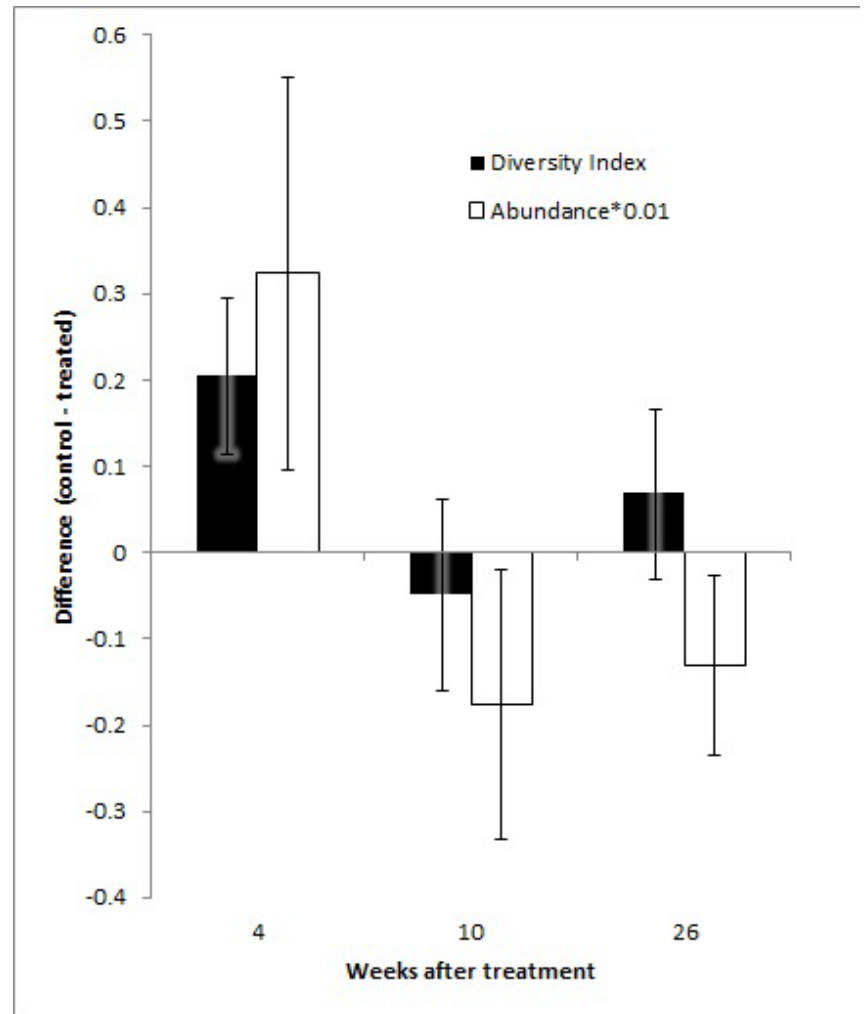

Figure 3. Average difference (control - treated plot) in Shannon diversity index and abundance (x0.01). Error bars show standard errors of the difference between two means.

\begin{tabular}{|c|c|c|c|c|c|c|c|c|c|c|c|c|}
\hline \multirow{2}{*}{$\begin{array}{c}\text { Time after } \\
\text { removal }\end{array}$} & \multicolumn{3}{|c|}{$\begin{array}{l}\text { Shannon-Weaver } \\
\text { Diversity Index }\end{array}$} & \multicolumn{3}{|c|}{ Abundance } & \multicolumn{3}{|c|}{ Species Richness } & \multicolumn{3}{|c|}{ Evenness } \\
\hline & Treated & Control & $p$ & Treated & Control & $p$ & Treated & Control & $p$ & Treated & Control & $p$ \\
\hline $\begin{array}{l}\text { Four } \\
\text { weeks }\end{array}$ & $\begin{array}{l}1.15 \pm \\
0.08\end{array}$ & $\begin{array}{c}1.35 \pm \\
0.06\end{array}$ & 0.0143 & $\begin{array}{c}127 \pm \\
17\end{array}$ & $\begin{array}{c}159 \pm \\
15\end{array}$ & 0.0258 & $\begin{array}{c}5.7 \pm \\
0.6\end{array}$ & $\begin{array}{c}6.5 \pm \\
0.8\end{array}$ & 0.415 & $\begin{array}{c}0.69 \pm \\
0.03\end{array}$ & $\begin{array}{c}0.77 \pm \\
0.04\end{array}$ & 0.144 \\
\hline $\begin{array}{c}\text { Ten } \\
\text { weeks }\end{array}$ & $\begin{array}{c}1.08 \pm \\
0.08\end{array}$ & $\begin{array}{c}1.03 \pm \\
0.08\end{array}$ & 0.568 & $\begin{array}{c}137 \pm \\
9\end{array}$ & $\begin{array}{c}119 \pm \\
13\end{array}$ & 0.0422 & $\begin{array}{c}7.0 \pm \\
0.8\end{array}$ & $\begin{array}{c}6.2 \pm \\
0.8\end{array}$ & 0.477 & $\begin{array}{c}0.57 \pm \\
0.02\end{array}$ & $\begin{array}{c}0.60 \pm \\
0.03\end{array}$ & 0.447 \\
\hline $\begin{array}{l}\text { Twenty-six } \\
\text { weeks }\end{array}$ & $\begin{array}{c}0.196 \pm \\
0.08\end{array}$ & $\begin{array}{c}0.265 \pm \\
0.06\end{array}$ & 0.252 & $\begin{array}{c}68 \pm \\
7\end{array}$ & $\begin{array}{c}55 \pm \\
8\end{array}$ & 0.0573 & $\begin{array}{c}2.1 \pm \\
0.3\end{array}$ & $\begin{array}{c}2.6 \pm \\
0.3\end{array}$ & 0.295 & $\begin{array}{c}0.202 \pm \\
0.06\end{array}$ & $\begin{array}{c}0.245 \pm \\
0.04\end{array}$ & 0.578 \\
\hline
\end{tabular}

Table 2. Shannon-Weaver diversity indices, abundance, species richness, and evenness, each \pm standard error of the mean, for treated and control plots after each survey shown with associated $t$ and $p$ values of two-tailed t-tests with 9 degrees of freedom. Bold values indicate statistical significance. 
Trends in treated plot recovery as represented by decreasing Euclidean distance

Before ivy removal, control and treated plots had an average Euclidean distance of 13.4. The greatest average Euclidean distance (44.2) was observed four weeks after ivy removal, and subsequent values decreased, first sharply (21.8 after 10 weeks), and then gradually (17.0 after 30 weeks; Figure 4).

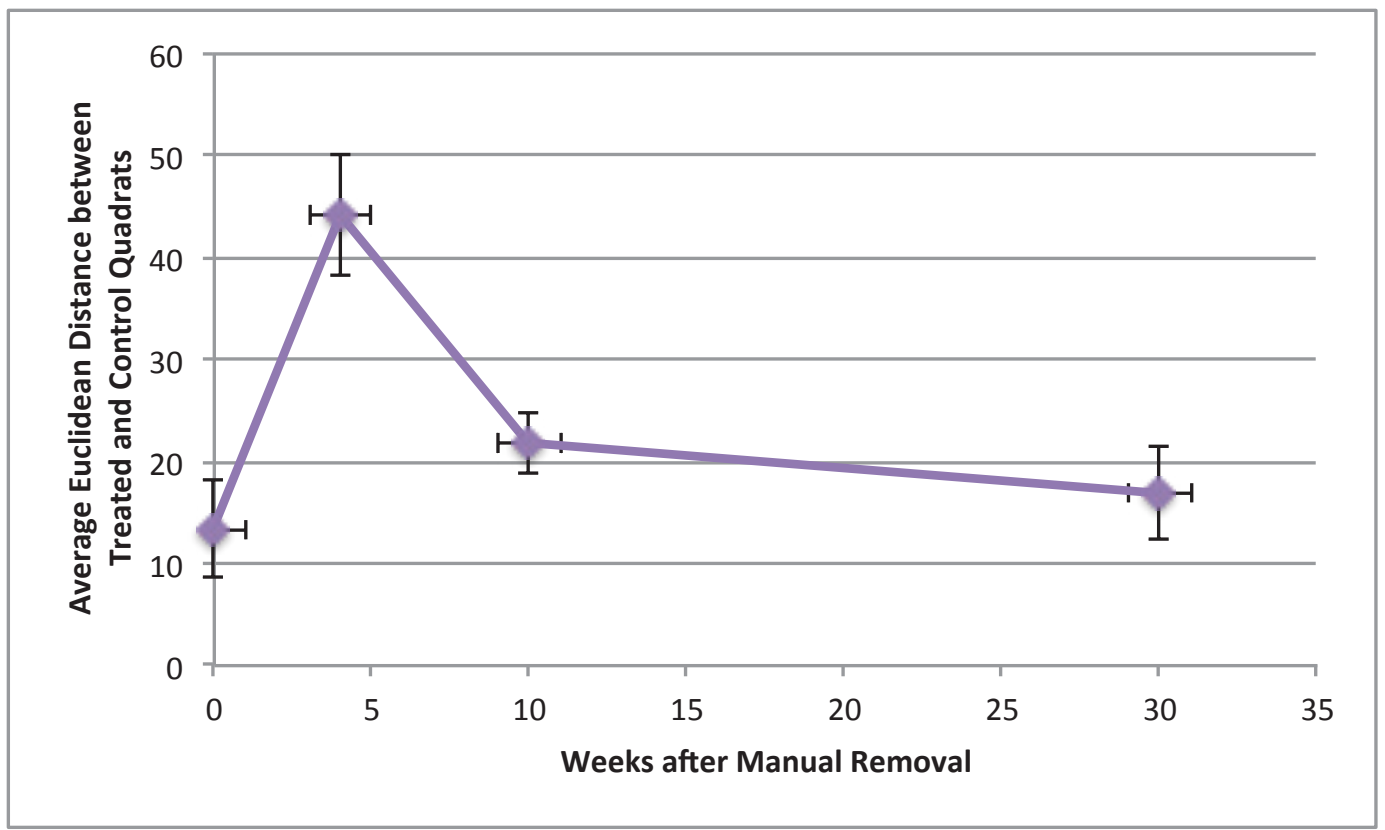

Figure 4. Euclidean distances between treated and control plots at each survey. The occurrence of a large initial dissimilarity followed by quick recovery is consistent with t-test data for plant diversity and abundance. Error bars show standard errors of the mean.

\section{DISCUSSION}

Four weeks after manual removal, treated plots were less diverse and hosted a lower abundance of native plants than control plots. After ten weeks, diversity indices of treated and control plots were no longer significantly different, and treated plots showed greater abundance of plant cover than controls. This trend persisted at twenty-six weeks after treatment. Treated and control plots were not significantly different in species richness or evenness (Table 2). These results suggest that the act of manual ivy removal temporarily disturbs plant life, but the negative effects are overcome as quickly as ten weeks after treatment, at which time a positive trend in native plant abundance may arise. Euclidean distance calculations confirmed the trend of an initial disturbance followed by quick recovery. Euclidean distance increased $230 \%$ four weeks after ivy removal, but decreased rapidly to approach within $30 \%$ of the initial distance after ten weeks (Figure 4). This suggests that the treatment initially increased dissimilarity between treated and control plots, but the effect decreased after ten and twenty-six weeks. Therefore, manual removal as a treatment method appears to be at least moderately long lasting and may allow further positive effects on native seedling recruitment.

It is also encouraging to note that, except for ivy, only native species emerged after ivy removal, though this may have been influenced by the location of the plots within established native communities and without close proximity to other exotic species. Furthermore, despite the dominance of ivy in the surrounding space, no ivy seedlings were observed within treated plots throughout the duration of the study. Thus, it appears ivy has either not established a dominant presence in the 
seed bank or that its seeds are not able to sprout sooner than native seeds. Even so, ivy was observed in treated plots after removal in the form of pioneering shoots, achieving an average of 12 percent cover by twenty-six weeks after removal. From the lack of ivy seedlings and the fact that ivy can establish subterranean roots from its shoots, ${ }^{24}$ vegetative reproduction appears to be the most significant contributing factor to the dominance of ivy in this region.

While the results of this experiment indicate that manual ivy removal has positive effects on native plant abundance after a period of at least ten weeks, it is not realistic to assume this is representative of the long-term effects of manual ivy removal. Ten weeks after treatment, many plants had not yet had the chance to sprout, nor had an entire cycle of seed dispersal-and thus seed bank formation - and germination of forest plants had been allowed to occur. Later in the season, after twenty-six weeks, the spring growth died off and the soil was dry and unfavorable for new plant growth. Accordingly, little plant life was observed other than ivy during the third survey (average of 68 abundance in test plots, in comparison to second survey average abundance of 137). An entire cycle of seeding, germination, and emergence was not reflected in this study, and thus diversity indices did not portray the total diversity of plant life in plots throughout the season, especially through the fall and winter months. It is postulated that more positive results, such as greater native plant diversity and abundance in cleared plots, would be likely to develop after a year or more of community recovery if ivy did not reclaim the cleared areas. Though prior studies have indicated that ivy does not inhibit seed bank formation, ${ }^{20}$ natural remediation time after treatment may be necessary to overcome the disruptive effects of pulling ivy to the soil and surrounding plant life.

Another limitation of this current data set is that many seedlings emerging during the surveys were only observed as a pair of cotyledons and were not identifiable. This led to a large collection of similar individuals being lumped into one or more "unknown" categories, which might have included more than one species. Indeed, some individuals placed in this category during the first survey showed signs of being Hydrophyllum tenuipes, but were not recorded as such due to the uncertainty of the determination. Fortunately, both of these issues affected control and treated plots equally, so they did not likely compromise the validity of the conclusions.

Despite these limitations, the results of this study support two conclusions. First, manual ivy removal is disruptive to the plant community, having immediate adverse effects on diversity and abundance of native species. Considering how deeply embedded the ivy lattices were within the soil in some treated plots, this result could be expected, for detritus, plant matter, and soil were greatly disturbed in the attempt to remove all parts of the ivy (Figure 2). However, the longevity of the negative consequences appears short-lived and may be overcome by the positive effects ivy eradication, which leads to the second important implication: while the native plant community may suffer initially, recovery of diversity and abundance is rather rapid. This is consistent with the hypothesis that native species are more successful in the absence of non-native species, especially those as invasive as ivy. The greater abundance of native plants in treated versus control plots after twenty-six weeks further supports the hypothesis that native plants benefit from ivy removal. Possible explanations for this trend include increased light, nutrient, and space availability for native plants.

Two additional observations from this study remain to be mentioned, the first of which being variance in moss growth patterns. Although the data indicate a similar percent cover by mosses in treated and control plots, mosses tended to form much fuller mats in control plots than in treated plots. Mosses growing in treated plots were characterized by numerous small, filamentous individuals. Full recovery of moss mats in treated plots was not observed during the study period, which presents qualitative evidence of the disruptive effects of manual ivy removal.

Secondly, deer tracks and droppings indicated that defoliation observed throughout the site, which encumbered plot pair establishment, was likely caused by deer. This implies that deer in this area of Forest Park have been using ivy as a winter food source, which in turn suggests that local deer are accustomed to hederin, a secondary metabolite in ivy that normally suppresses herbivore appetite. ${ }^{18}$ It is possible that this browsing damages ivy individuals in such a way that their growth 
may be hindered, or that defoliation followed by vegetative growth of surviving ivy may be contributing to the mesh-like network of ivy growth throughout the understory if new shoots grow over the older, defoliated woody stems. In either case, further investigation into the relationship of ivy to native herbivores is warranted.

Other future avenues of research include studying the effect of manual ivy removal on seedling recruitment in a different location in Forest Park. Likely because of the moist environment, the current site was characterized by an abundance of Hydrophyllum tenuipes, which itself may rapidly overshadow any slower-growing species. Setting up plots in a different location, perhaps nearer to established populations of local invasive species such as Geranium robertianum (herb Robert) or Rubus bifrons (Himalayan blackberry), would also be useful to determine whether successive invasion by nonnative species presents an obstacle for recovery after ivy removal. Moreover, the principal cause of ivy dominance in the PNW has yet to be determined and is instead often speculatively inferred. Like other invasive species, there are many possible mechanisms by which ivy may be achieving dominance in PNW forests, such as light competition, nutrient competition, allelopathy, or alteration of soil microbial composition. ${ }^{26}$ Understanding the underlying cause of ivy invasion may advance the development of an effective solution.

\section{CONCLUSION}

Given the archetypical pattern of ivy invasion in Forest Park relative to much of the PNW, ${ }^{25}$ this study has important implications for ivy control throughout the region. The results support the use of manual ivy removal to promote native plant success, though care should be taken to minimize initial habitat disturbance, although prevention may remain the ideal solution to the problem of ivy in the PNW.

\section{ACKNOWLEDGMENTS}

I thank Portland Parks and Recreation, especially natural resources ecologist Kendra PetersenMorgen, for permitting research at this site. Thanks also go to Dr. Christine Weilhoefer for methodological expertise and Blair Pearson for assistance in conducting field work.

\section{REFERENCES}

[1] Jones, C. and Reichard, S. (2009) Current and potential distributions of three non-native invasive plants in the contiguous USA, Nat Area J, 29(4), 332-343.

[2] USDA, NRCS (2013) The PLANTS Database, National Plant Data Team, http://plants.usda.gov/ core/profile?symbol=HEHE (accessed Nov 2013).

[3] Metcalfe, D. (2005) Hedera helix L., J Ecol, 93, 632-648.

[4] Yaman, B. (2009) Comparative wood anatomy of ivy-hosting and non-hosting oriental plane (Platanus orientalis L.), Plant Biosyst, 143(2), 252-257.

[5] Soll, J. (2005) Controlling English ivy (Hedera helix) in the Pacific Northwest, Global Invasive Species Team, The Nature Conservancy, http://www.invasive.org/gist/moredocs/hedhel02.pdf (accessed Feb 2015).

[6] Van Cowenberghe, R., Collet, C., Lacombe, E., and Gegout, J. (2011) Abundance response of western European forest species along canopy openness and soil pH gradients, Forest Ecol Manag, 262, 1483-1490.

[7] Dlugosch, K. (2005) Understory community changes associated with English ivy invasions in Seattle's urban parks, Northwest Sci, 79(1), 53-60.

[8] Thomas, L. K. (1980) Impact of three exotic plant species on a Potomac Island, Clemson University Libraries, [Online], 58-65 (accessed May 2014). 
[9] Shoup, S. and Whitcomb, C. E. (1981) Interactions between trees and ground covers, J Arboric, 7(7), 186-187.

[10] Reichard, S. (2000) Hedera helix, in Invasive Plants of California's Wildlands, (C. C. Bossard, J. M. Randall, and M. C. Hoshovsky, Eds.) pp. 212-215, University of California Press, Berkeley, CA.

[11] Okerman, A. (2000) Combating the "ivy desert": the invasion of Hedera helix (English ivy) in the Pacific Northwest United States, Restoration and Reclamation Review - Student Online Journal, 6(4), http://conservancy.umn.edu/bitstream/handle/11299/59738/6.4. Okerman.pdf?sequence=1 (accessed Feb 2015).

[12] Jones, C. C., Acker, S. A., and Halpern, C. B. (2010) Combining local- and large-scale models to predict the distributions of invasive plant species, Ecol Appl, 20(2), 311-326.

[13] Vidra, R., Shear, T., and Wentworth, T. (2006) Testing the paradigms of exotic species invasion in urban riparian forests, Nat Area J, 26(4), 339-350.

[14] Chapin, S., Walker, B., Hobbs, R., Hooper, D., Lawton, J., Sala, O., and Tilman, D. (1997) Biotic control over the functioning of ecosystems, Science, 277, 500-504.

[15] Derr, J. F. (1993) English ivy (Hedera helix) response to postemergence herbicides, J Environ Hortic, 11(2), 45-48.

[16] Yang, Q., Wehtje, G., Gilliam, C., McElroy, J., and Sibley, J. (2013) English ivy (Hedera helix) control with postemergence-applied herbicides, IPSM, 6(3), 411-415.

[17] Swearingen, J. and Diedrich, S. (2006) English Ivy (Hedera helix L.) Plant Conservation Alliance's Alien Plant Working Group, http://www.nps.gov/plants/alien/fact/hehe1.htm (accessed Feb 2015).

[18] Ingham, C. and Borman, M. (2010) English ivy (Hedera spp., Araliaceae) response to goat browsing, IPSM, 3(2), 178-181.

[19] Young, S., Simmons, R., and Hamblin-Katnik, C. (2012) Instructions for removing English ivy and discussion of safety, City of Alexandria Dept. Recreation, Parks, and Cultural Activities, Alexandria, Virginia, http://alexandriava.gov/48838 (accessed Nov 2014).

[20] Biggerstaff, M. S. and Beck, C. W. (2007) Effects of English ivy (Hedera helix) on seed bank formation and germination, Am Midl Nat, 157, 250-257.

[21] Bauerle, T. and Chalker-Scott, L. (2001) Relative effectiveness of control mechanisms for juvenile English ivy (Hedera helix) as measured by leaf respiration and root-crown starch content, Hortscience, 36(3), 431

[22] Pojar, J. (2004) Plants of the Pacific Northwest Coast, revised ed. Lone Pine Publishing, Auburn, WA.

[23] Hitchcock, C. L. (1973) Flora of the Pacific Northwest: An Illustrated Manual. University of Washington Press, Seattle, WA.

[24] Melzer, B., Seidel, R., Steinbrecher, T., and Speck, T. (2011) Structure, attachment properties, and ecological importance of the attachment system of English ivy (Hedera helix), J Exp Bot, [Online], err260v1-err260.

[25] City of Portland, Oregon. (2014) The Ivy Files, Parks and Recreation, https://www.portlandoregon.gov/parks/47820 (accessed May 2014).

[26] Bennett, A., Thomsen, M., and Strauss, S. (2011) Multiple mechanisms enable invasive species to suppress native species, Am J Bot, 98(7), 1086-1094. 


\section{ABOUT THE STUDENT AUTHOR}

Katelin D. Stanley is a recent biology graduate from the University of Portland in Portland, Oregon and is currently a Ph.D. student at the Florida State University. Having had the opportunity to participate in several research projects during her undergraduate years, Katelin is eager to pursue a future in ecological and biodiversity research. Her scientific interests include ecology and plant systematics, with special emphasis on the use of biological databases such as herbaria.

\section{PRESS SUMMARY}

The two exotic vine species English and Irish ivy (Hedera helix and $H$. hibernica, respectively) have invaded natural areas in the Pacific Northwest, demonstrating rampant growth and rapid climbing ability. The most common strategy for ivy control is currently removal with clippers and by hand, though the potential consequences of this method have not been well studied. The purpose of this study was to evaluate the effectiveness of this method by investigating its effects on native plant life. Results indicate that manual removal is at first disruptive to native plants, but the negative effects decrease after ten weeks and are replaced by greater native plant abundance. This supports the use of manual ivy removal to control ivy invasions.

\section{AJUR Volume 12 | Issue 4 | November 2015}




\title{
Imaging Noisy Seismic Data using a One Dimensional Inverse Scattering Algorithm
}

\author{
Bogdan G. Nita \& Christopher Smith \\ Department of Mathematical Sciences, Montclair State University, Montclair, NJ \\ Student: chris.smith324@gmail.com \\ Mentor:nitab@mail.montclair.edu
}

\begin{abstract}
We test the capability of an inverse scattering algorithm for imaging noisy seismic data. The algorithm does not require a velocity model or any other a priori information about the medium under investigation. We use three different geometries which capture different types of one-dimensional media with variable velocity. We show that the algorithm can precisely locate the interfaces and discover the correct velocity changes at those interfaces under moderate noise condition. When the signal to noise ratio is too small, the data is de-noised using a threshold filter and then imaged with excellent results.
\end{abstract}

\section{KEYWORDS}

Seismic Imaging, Inversion, Amplitude Correction, Scattering Theory, Noise, Threshold Filter. 2000 MATHEMATICS SUBJECT CLASSIFICATION 86A22, 35J05, 35R30.

\section{INTRODUCTION}

Inverse scattering theory is a framework for determining the characteristics of an object from measurement data of waves or particles scattered from that object. In contrast to other imaging methods, no a priori information about the object or about the medium surrounding the object is necessary to extract the location and the characteristics of the object. This makes inverse scattering theory a very unique tool, presently being the only direct method with this capability. The application of inverse scattering methods to seismic exploration has been extensively discussed in the literature (see for example [1] and [2] and the references therein). ${ }^{1,2}$ In 2009, Nita $^{3}$ found an inverse scattering algorithm for simultaneous imaging and inversion which was recently tested numerically with excellent results. ${ }^{4}$

The data recorded in a seismic experiment contain several types of arrivals like primary reflections, multiple reflections, free surface multiple reflections, source and receiver ghost waves, direct wave from the source, ambient noise and others depending on the location of the receivers. ${ }^{6}$ Out of these, only primary reflections are considered useful signal and all current imaging algorithms are designed to process only this small part of the data. ${ }^{7}$ Any other type of signal is considered noise and consequently eliminated, totally or partially, in a pre-processing phase.

The unwanted part of the data can be further split into two categories: coherent and random noise. Coherent noise usually consists of multiple reflections of the initial wave and many algorithms exist today to attenuate or eliminate it. ${ }^{8}$ In this paper, we test the capability of the inverse scattering algorithm using data contaminated with random noise. We use various earth models to capture the characteristics of several earth configurations (different number of layers, different velocity contrasts and velocity inversions). For consistency and comparison purposes we use the same models as did Tasy. ${ }^{4}$ Following the standard seismic industry pre-processing steps, we assume that the source signature has been deconvolved from the data and that all coherent noise has been eliminated. Therefore, the data that we use in our imaging algorithm only consists of single (primary) reflections and random noise. 


\section{BACKGROUND}

In this section we briefly describe the theory behind the algorithm that we will be testing. We start with the equation describing the acoustic wave propagation in a 1-D constant density variable velocity medium

$$
\left(\frac{d^{2}}{d z^{2}}+k^{2}(z)\right) P(z, \omega)=0 \quad \text { Equation } 2.1
$$

where $P$ is the pressure field at depth $z, k(z)=\omega / c(z)$ is the vertical wavenumber, $\omega$ the angular frequency and $c(z)$ the velocity of sound in the medium. This equation does not include a source term and hence it only models the propagation of an existing waveform and not how the waveform is created. Assume a reference medium represented by an acoustic wholespace with velocity $c_{0}$, and define a perturbation operator ${ }^{1}$

$$
V=k_{0}^{2} \alpha(z) \quad \text { Equation } 2.2
$$

where $k_{0}=\frac{w}{c_{0}}$ and $\alpha(z)=1-\frac{c_{0}^{2}}{c^{2}(z)}$. In this framework, the inverse problem is to solve for $\alpha$ which in turn will provide information about the velocity in the unknown medium, $c(z)$. The inverse scattering series is a power series containing powers of the collected data and, for this problem (one dimensional medium with one parameter - velocity), it takes the form

$$
\alpha(z)=\alpha_{1}(z)+\alpha_{2}(z)+\alpha_{3}(z)+\ldots \quad \text { Equation } 2.3
$$

where $\alpha_{i}(z)$ for any $i \geq 1$ contains the $i-t h$ power of the data.

After solving for the first few terms in the series, and identifying them as either imaging or inversion driving term $\mathrm{s}^{5}$ one can select the desired pieces and group them in a subseries which only performs a targeted task: free surface or internal multiple attenuation, imaging or inversion. Such a subseries for simultaneous imaging and inversion was discovered ${ }^{3}$ which showed that the respective series is convergent for all values of the perturbation operator and the limit is,

$$
\alpha^{S I I}(z)=\int_{-\infty}^{\infty} e^{i k_{0} z} \int_{-\infty}^{\infty} \alpha_{1}\left(z^{\prime}\right) e^{-i k_{0}\left(z^{\prime}+\frac{1}{2} \int_{-\infty}^{z^{\prime}} \alpha_{1}\left(z^{\prime \prime}\right) d z^{\prime \prime}\right)} d z^{\prime} d k_{0} .
$$

Equation 2.4

This is the closed form of the subseries for imaging and inversion which will be tested numerically in this paper with noisy seismic data. As mentioned before, the algorithm assumes that multiples have been removed from the data (in addition to source signature and ghosts) and therefore the data only contains primaries.

Although Equation 2.4 describes only a 1-D algorithm, this algorithm promises the recovery of an image of the actual medium from collected data and knowledge of a reference medium only, without a velocity model or any other assumption about the medium under investigation. All other seismic exploration imaging techniques are dependent on some a priori assumption about the targeted medium. ${ }^{9}$ 


\section{NUMERICAL TESTS FOR THE SIMULTANEOUS IMAGING AND INVERSION ALGORITHM}

In this chapter we test the Simultaneous Imaging and Inversion Algorithm using several data sets collected over three earth models. All numerical tests were performed on a Desktop PC using MAPLE. For each earth model we have to first create the geometry (layers and corresponding velocities), then simulate a seismic experiment to create the data, and finally corrupt this data with random noise. The data is then ran through the imaging algorithm (Equation 2.4) and the image is compared with the initial model. We will observe two main characteristics of the algorithm: its ability to correctly find the depths of the interfaces of the unknown media and its ability to determine the correct amplitudes in the perturbation operator.

\subsection{MODEL 1: MONOTONIC INCREASING VELOCITY}

The first model consists of three interfaces located in water at depths of $z_{1}=100, z_{2}=130$, and $z_{3}=160$ with the sound velocity inside of the layers having the values $c_{0}=1500, c_{1}=1650, c_{2}$ $=1725$, and $c_{3}=1800$ (see Figure 1a). The perturbation operator, $\alpha$, for this earth model is shown in Figure 1b. The data in this case consists of three primary reflections shown in Figure 1c. The output of the algorithm is shown in blue in Figure 1d and it is easily compared with the actual model (in red) and with the first approximation $\alpha_{1}$ in green.

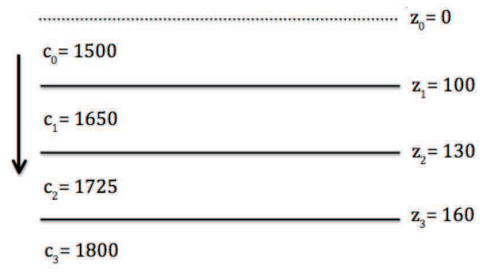

(a) Earth Model 1

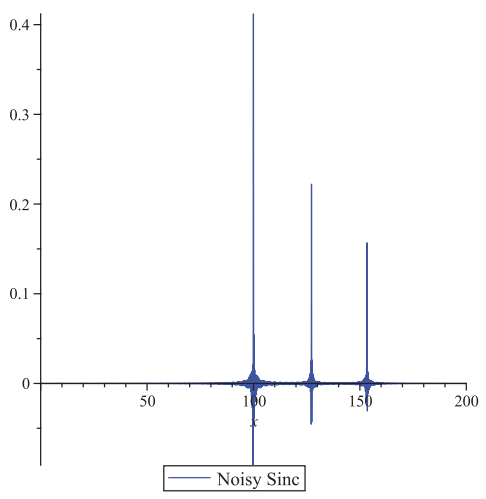

(c) Model 1 no noise data

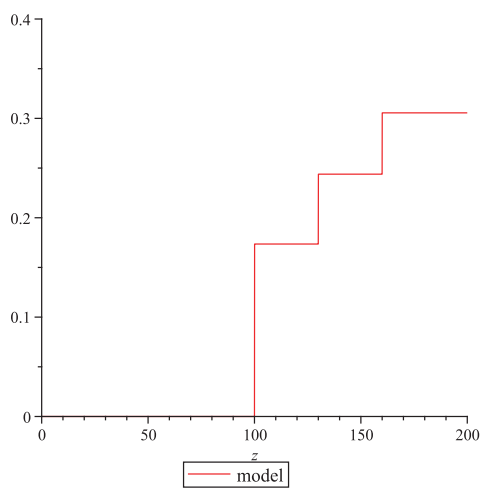

(b) Perturbation Operator for Model 1

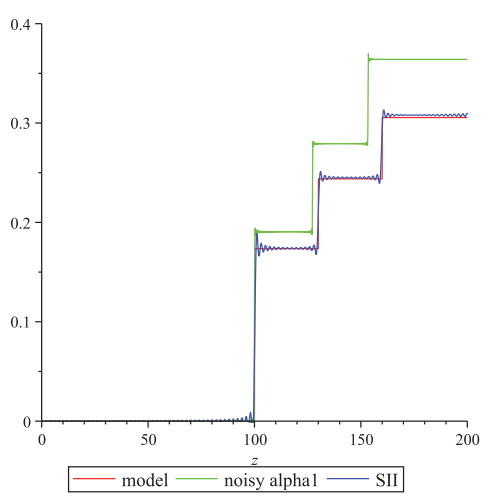

(d) Model 1 no noise image

Figure 1. Model 1 imaging algorithm using noise free data 
We incrementally add noise to the data (with a standard deviation STD ranging from 0.001 to 0.01 ), see Figures $\mathbf{2} \mathbf{a}, \mathbf{2} \mathbf{c}, \mathbf{2} \mathbf{e}$ and notice how the image produced by the algorithm deteriorates in Figures 2b, 2d, $2 f$.

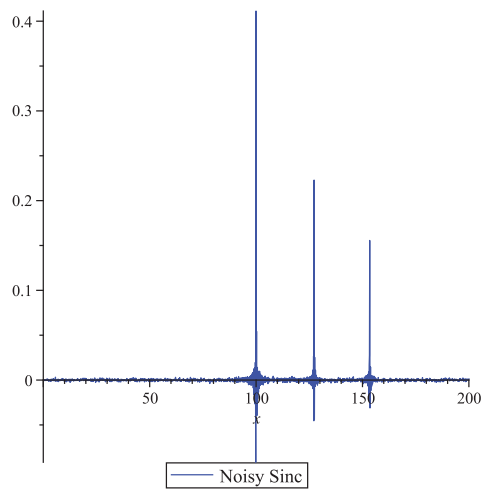

(a) Model 1 STD 0.001 noise data

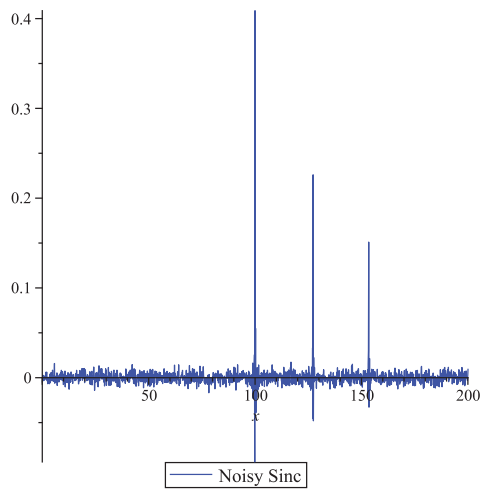

(c) Model 1 STD 0.005 noise data

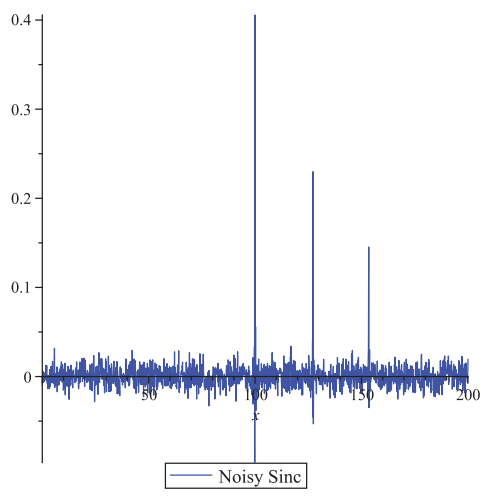

(e) Model 1 STD 0.01 noise data

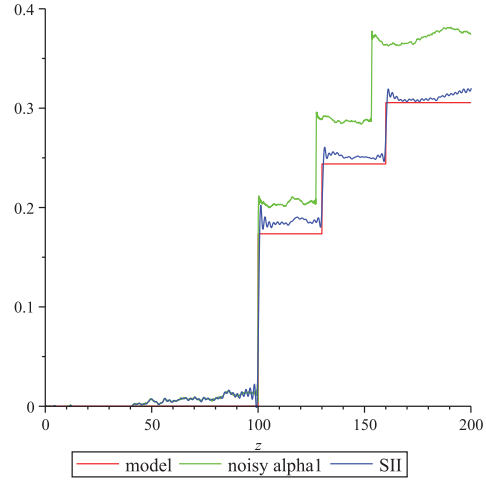

(b) Model 1 STD 0.001 noise image

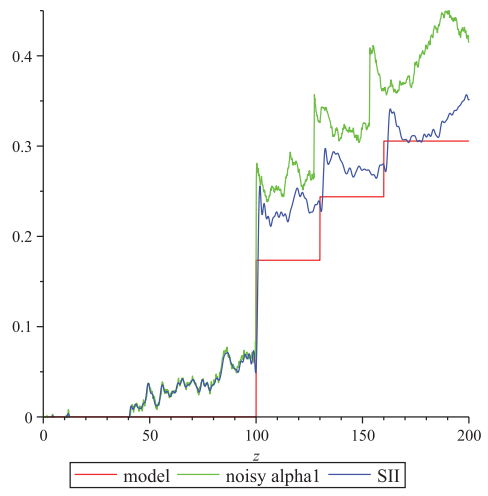

(d) Model 1 STD 0.005 noise image

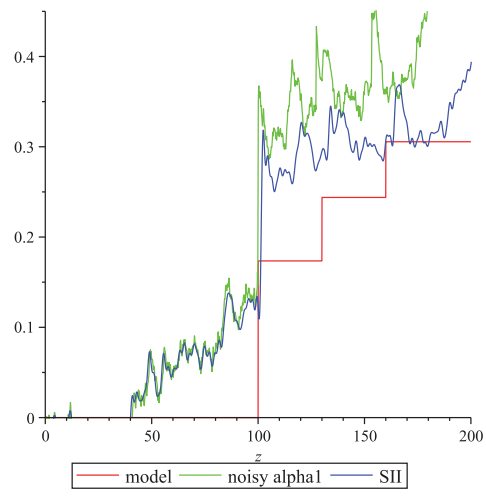

(f) Model 1 STD 0.01 noise image

Figure 2. Model 1 imaging algorithm with increasingly more noise

Not surprisingly, the high level of random noise in the data affects the image negatively. Following the common seismic processing practice, we proceed by applying a filter to the data to clean up some of this noise. There are many types of filters that can be used to attenuate or eliminate random 
noise. For example one can use non-causal prediction filters, ${ }^{10}$ adaptive filters ${ }^{11}$ or some transform methods like seislet transform, ${ }^{12}$ discrete cosine transform ${ }^{13}$ and curvelet transform ${ }^{14}$ to improve the signal to noise ratio. Due to the nature of this project, we decided on a simpler threshold filter which practically mutes all signal with an amplitude less than 0.05 (see Figures 3a, 3c, 3e). We then use the algorithm on the filtered data and obtain the images shown in Figures $\mathbf{3 b}, \mathbf{3 d}, \mathbf{3 f}$.
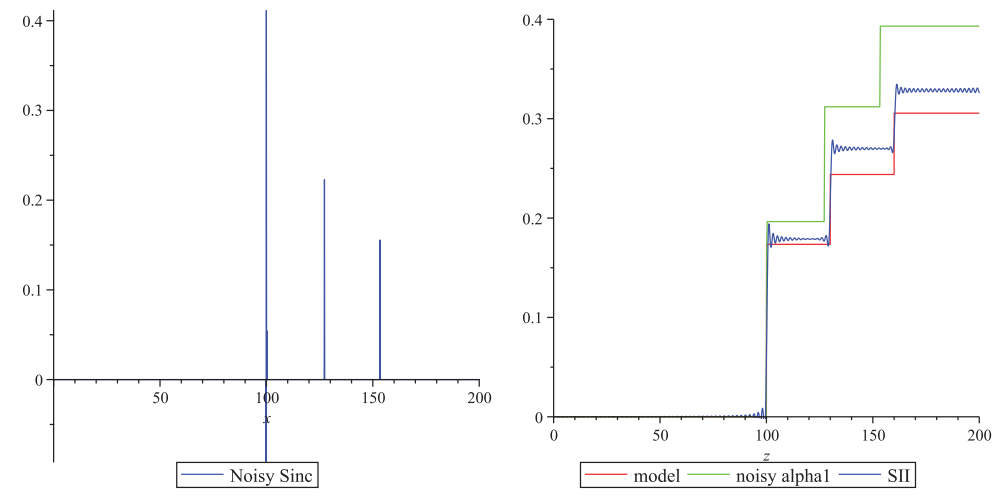

(a) Model 1 STD 0.001 noise filtered data(b) Model 1 STD 0.001 noise filtered im-

age
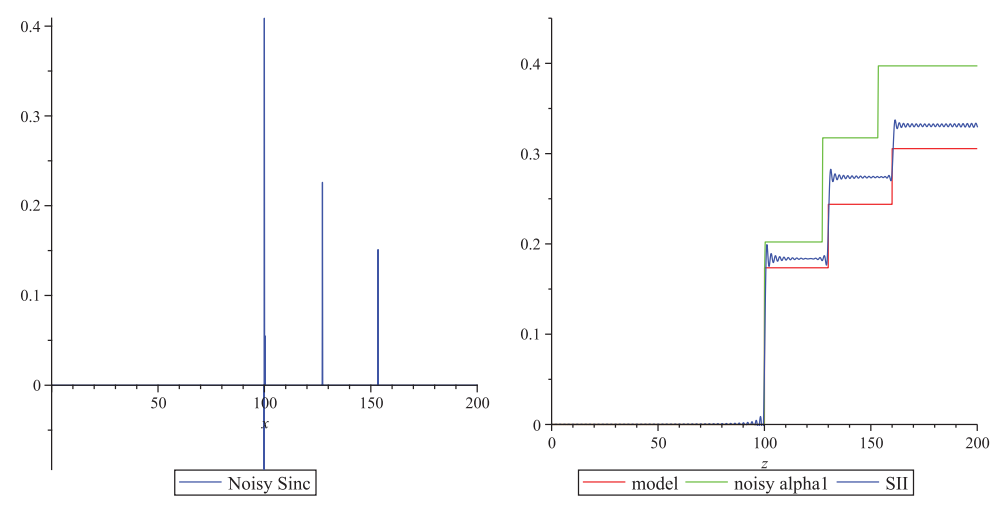

(c) Model 1 STD 0.005 noise filtered data(d) Model 1 STD 0.005 noise filtered image
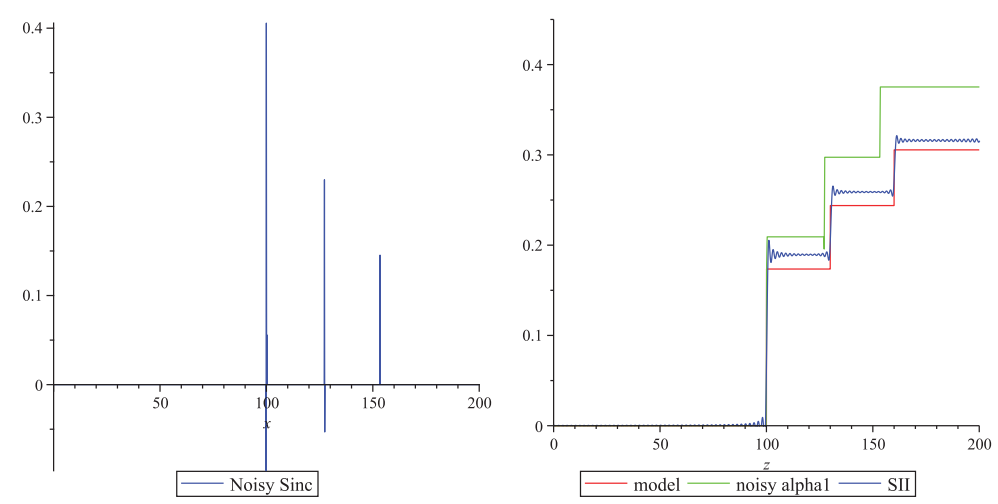

(e) Model 1 STD 0.01 noise filtered data (f) Model 1 STD 0.01 noise filtered image

Figure 3. Model 1 imaging algorithm with increasingly more noise 
The obtained results show that the algorithm is stable and capable of reproducing the correct location of the interfaces even after significant noise contamination. We notice that some amplitude information is lost due to the noise and the applied filter; however this could be improved by using a more complex filtering method.

\subsection{MODEL 2: NON-MONOTONIC VELOCITY}

The second model consists of four interfaces located in water at depths of $z_{1}=100, z_{2}=130$, $z_{3}=160$ and $z_{4}=200$ with the sound velocity inside of the layers having the values $c_{0}=1500, c_{1}=1650$, $c_{2}=1725, c_{3}=1575$ and $c_{4}=1725$ (See Figure 4a). This model is important to examine because, unlike the first example, the velocities in the layers are no longer monotonic. The perturbation operator

for this earth model is shown in Figure 4b. The data in this case consists of four primary refl ections shown in Figure 4c. Notice that the third spike in the data, corresponding to the primary reflection off the third interface, is negative. This is because, at the third interface, the velocity in the deeper layer is less then the velocity in the shallower layer. The output of the algorithm is shown in blue in Figure $\mathbf{4} \mathbf{d}$ together with the actual model (in red) and with the first approximation $\alpha_{1}$ in green.

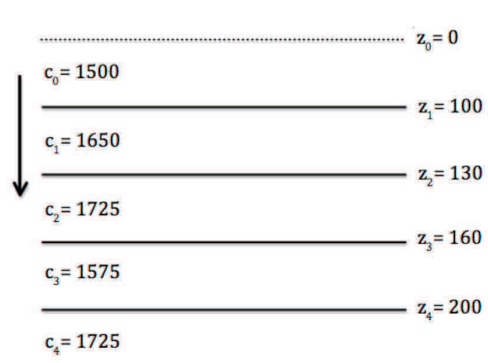

(a) Earth Model 2

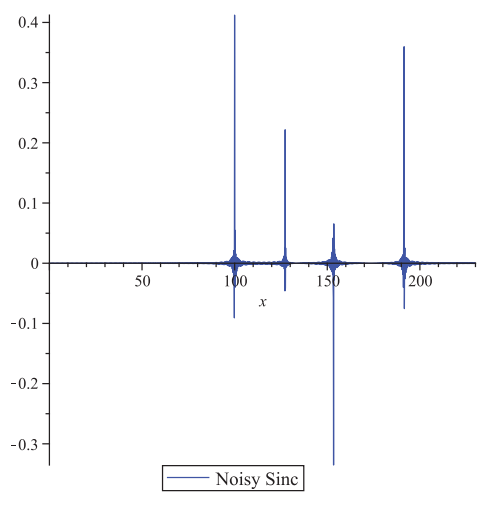

(c) Model 2 no noise data

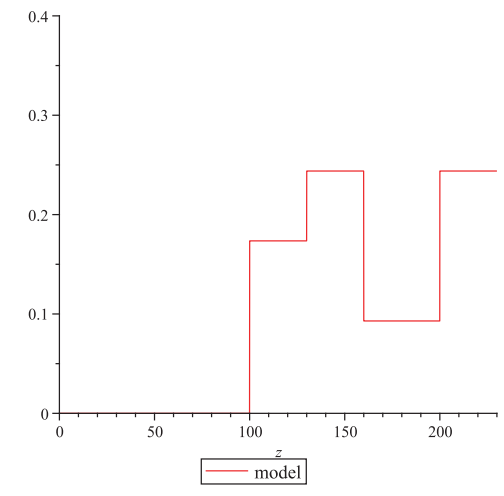

(b) Perturbation Operator for Model 2

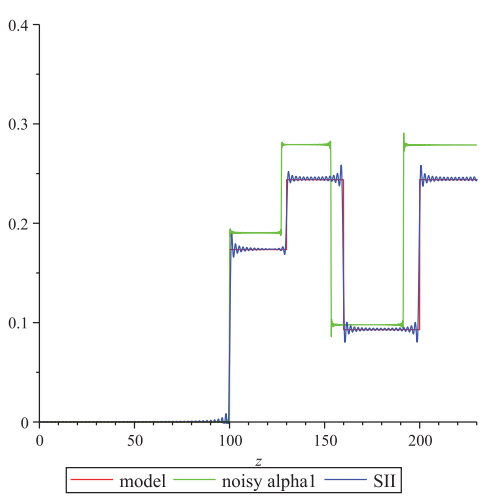

(d) Model 2 no noise image

Figure 4. Model 2 imaging algorithm using noise free data 
As before we add noise to the data, with a standard deviation ranging from 0.001 to 0.01 (see Figures $\mathbf{5 a}, \mathbf{5 c}, \mathbf{5 e}$ ). As expected, the image produced by the algorithm becomes distorted and makes it very difficult to extract any useful information from it (see Figures $\mathbf{5 b}, \mathbf{5 d}, \mathbf{5 f}$ ).

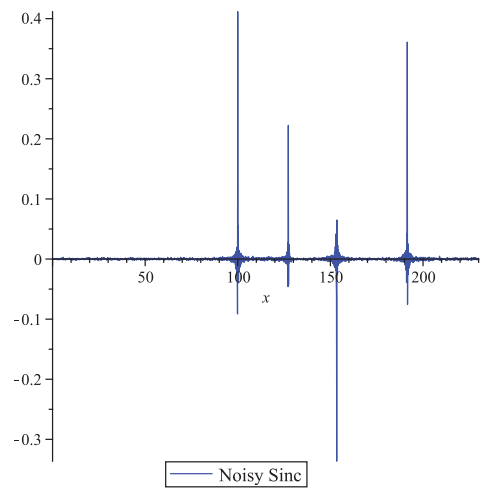

(a) Model 2 STD 0.001 noise data

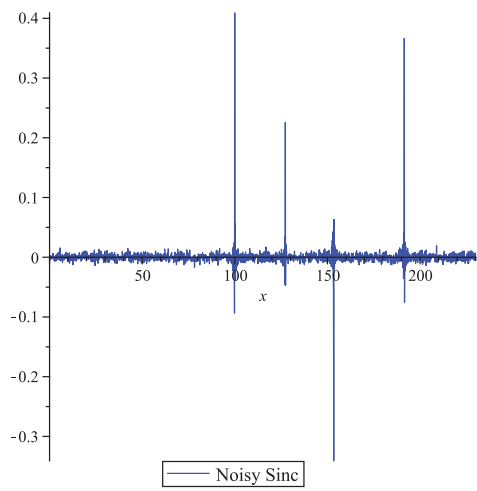

(c) Model 2 STD 0.005 noise data

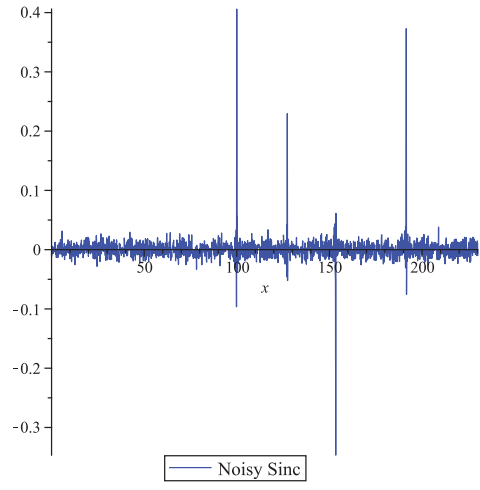

(e) Model 2 STD 0.01 noise data

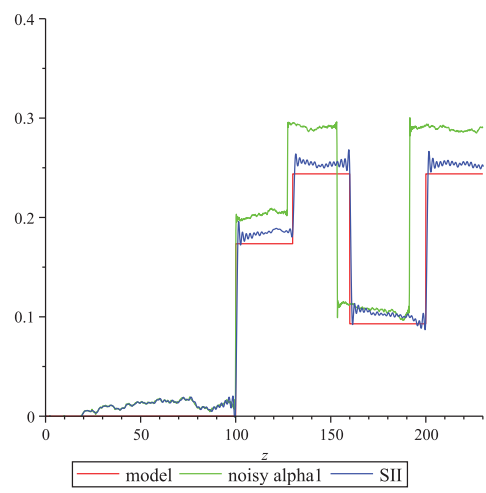

(b) Model 2 STD 0.001 noise image

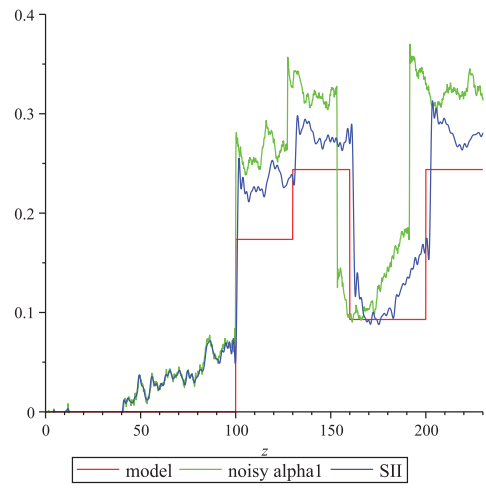

(d) Model 2 STD 0.005 noise image

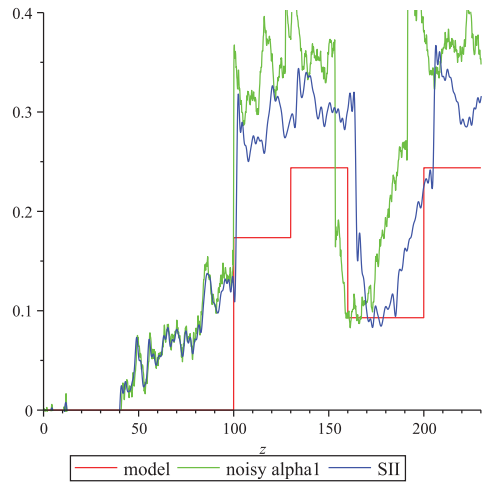

(f) Model 2 STD 0.01 noise image

Figure 5. Model 2 imaging algorithm with increasingly more noise 
After applying the same threshold filter as before (see Figures $\mathbf{6 a}, \mathbf{6 c}, \mathbf{6 e}$ ), the image clears up and, although some amplitude information is lost, the interfaces of the model are clearly delineated for all three levels of noise (see Figures $\mathbf{6 b}, \mathbf{6 d}, \mathbf{6 f}$ ). In conclusion, for this second model, the combination of data filtering and inverse scattering algorithm works very well.
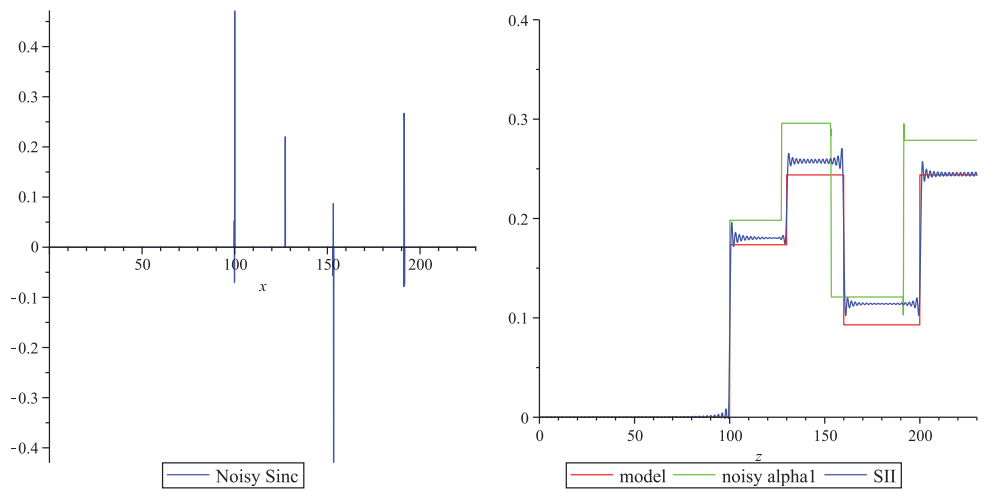

(a) Model 2 STD 0.001 noise filtered data(b) Model 2 STD 0.001 noise filtered image
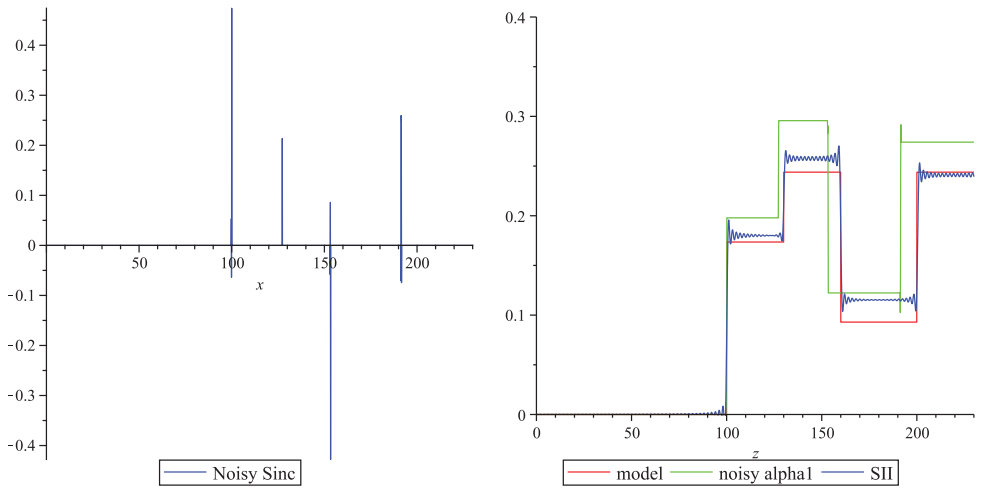

(c) Model 2 STD 0.005 noise filtered data(d) Model 2 STD 0.005 noise filtered image
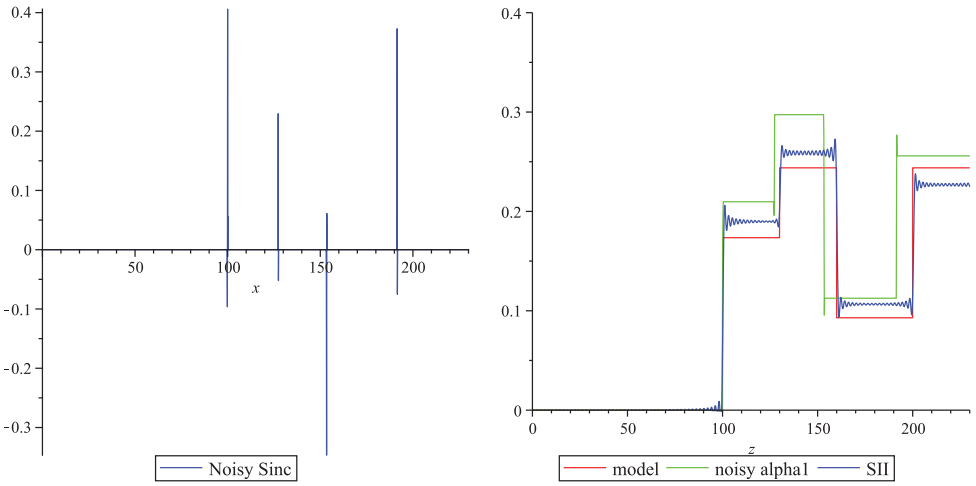

(e) Model 2 STD 0.01 noise filtered data (f) Model 2 STD 0.01 noise filtered image

Figure 6. Model 2 imaging algorithm with increasingly more noise 


\subsection{MODEL 3: OSCILLATING VELOCITY}

The third model consists of six interfaces located in water at depths of $z_{1}=100, z_{2}=130, z_{3}=$ $160, z_{4}=200, z_{5}=240$, and $z_{6}=260$ with the sound velocity inside of the layers having the values $c_{0}=1500$ and then alternating between 1850 and 1625, respectively (see Figure 7a). This model is important to examine because it contains several velocity inversions and large velocity contrasts. The perturbation operator for this earth model is shown in Figure $\mathbf{7 b}$. The data in this case consists of six primary reflections plotted in the depth domain in Figure 7c. Notice that the pulses alternate between positive and negative amplitude, which is consistent with the alternating velocity inversions in the model. The output of the algorithm is shown in blue in Figure $7 \mathrm{~d}$ together with the actual model (in red) and with the first approximation $\alpha_{1}$ in green.

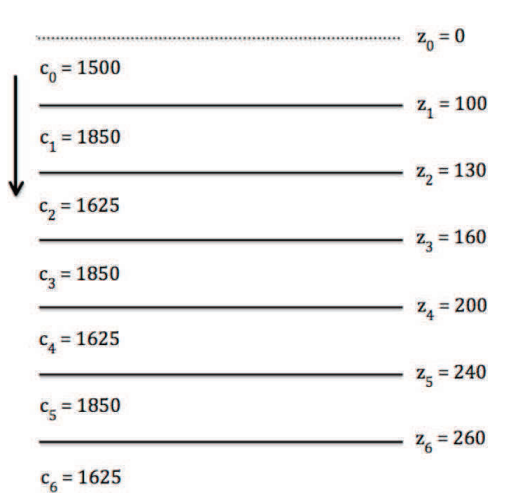

(a) Earth Model 3

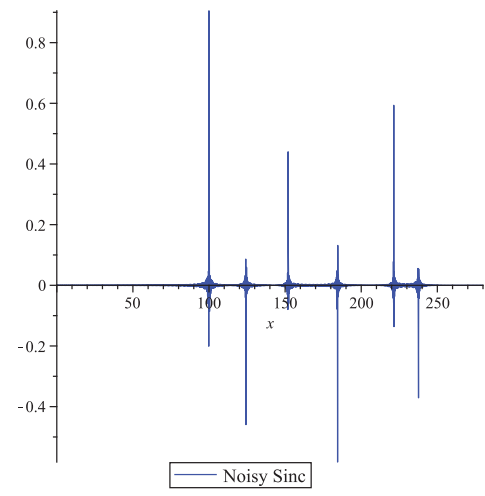

(c) Model 3 no noise data

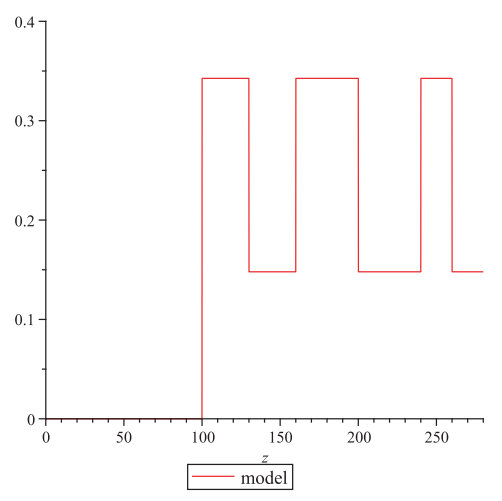

(b) Perturbation Operator for Model 3

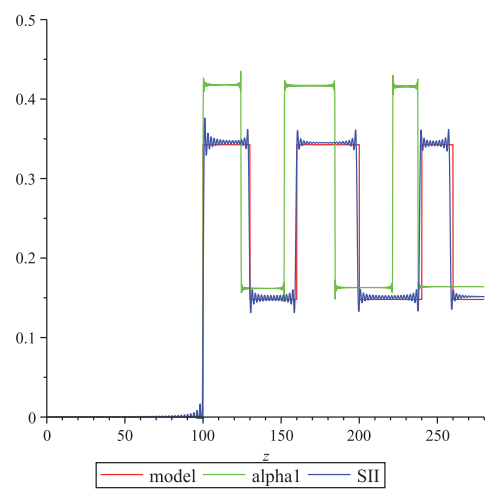

(d) Model 3 no noise image

Figure 7. Model 3 imaging algorithm using noise free data 
In the next step we add noise to the data, with the same standard deviation as before (see Figures $\mathbf{8 a}, \mathbf{8 c}, \mathbf{8 e}$ ). The image produced by the algorithm deteriorates accordingly as it can be seen in Figures $8 b, 8 d, 8 f$.

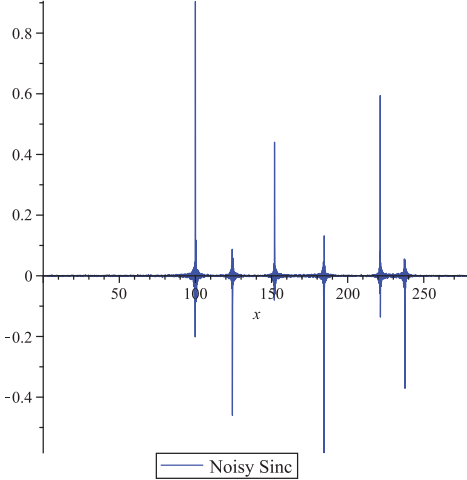

(a) Model 3 STD 0.001 noise data

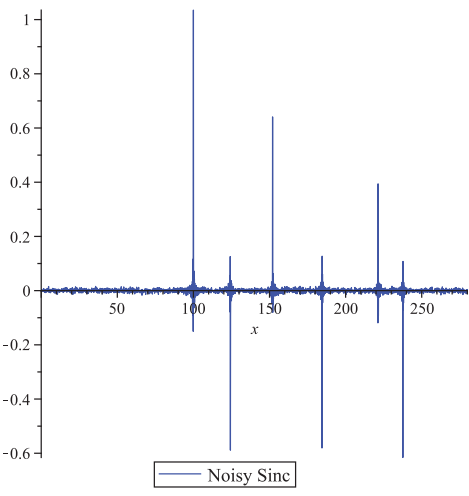

(c) Model 3 STD 0.005 noise data

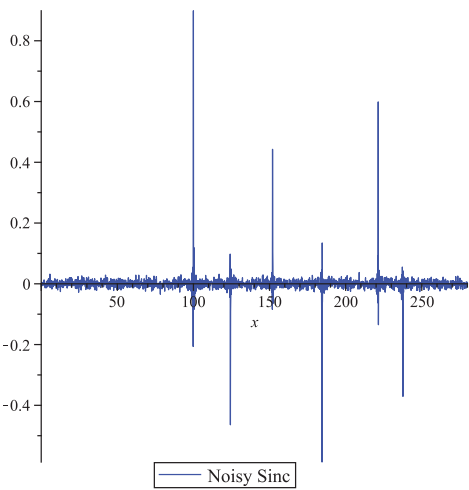

(e) Model 3 STD 0.01 noise data

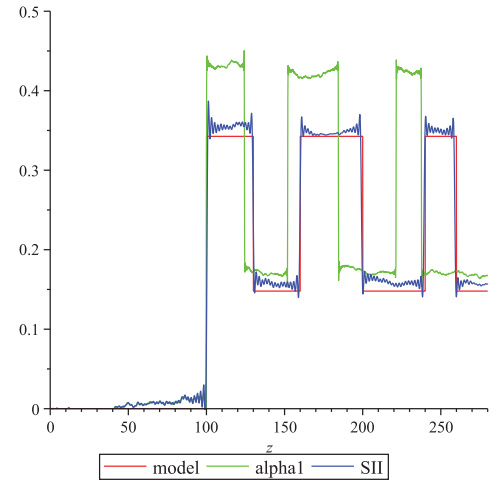

(b) Model 3 STD 0.001 noise image

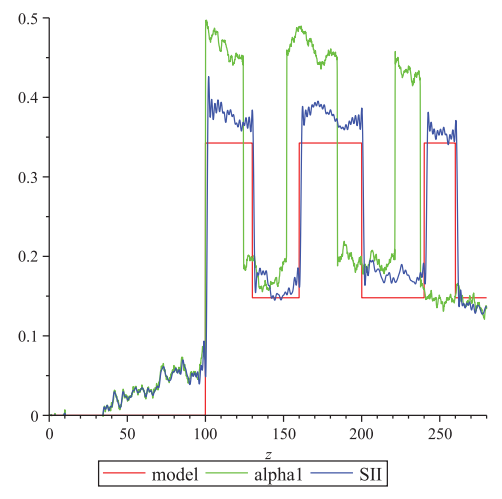

(d) Model 3 STD 0.005 noise image

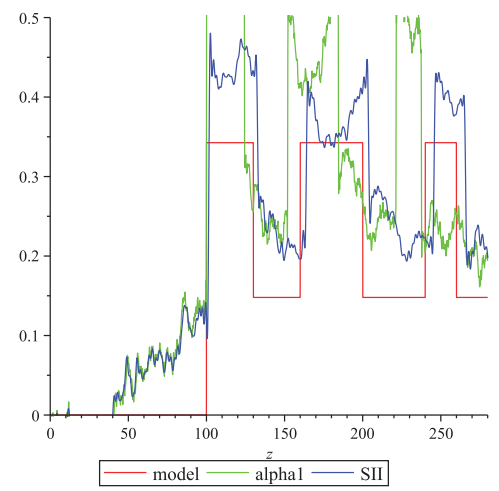

(f) Model 3 STD 0.01 noise image

Figure 8. Model 3 imaging algorithm with increasingly more noise 
Filtering the noise using the same threshold filters cleans up the data as seen in Figures $9 a, 9 c$, 9ebut it also results in some loss of amplitude information in the final image (see Figures $9 \mathbf{b}, \mathbf{9 d}, \mathbf{9 f}$ ). We notice however that the algorithm places all interfaces at their exact location.
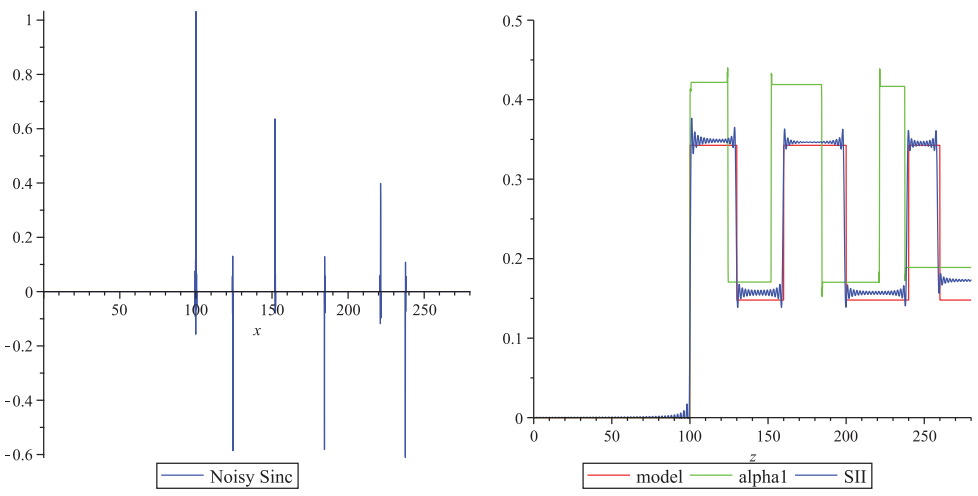

(a) Model 3 STD 0.001 noise filtered data(b) Model 3 STD 0.001 noise filtered image
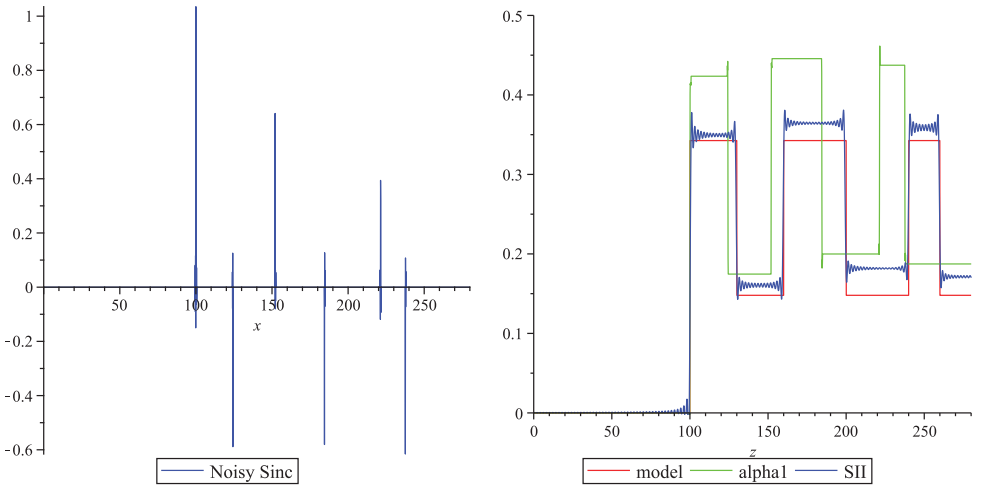

(c) Model 3 STD 0.005 noise filtered data(d) Model 3 STD 0.005 noise filtered image
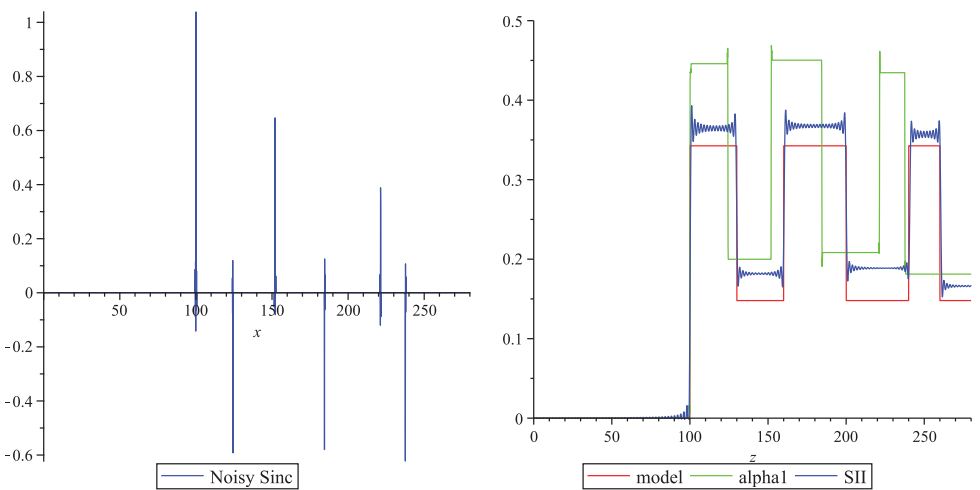

(e) Model 3 STD 0.01 noise filtered data (f) Model 3 STD 0.01 noise filtered image

Figure 9. Model 3 imaging algorithm with increasingly more noise 


\section{CONCLUSION}

In this paper, we tested the capability of an inverse scattering algorithm for imaging seismic data. The algorithm we investigated simultaneously images and inverts one-dimensional, one-parameter (velocity), acoustic reflection data. The algorithm does not require a velocity model or any other a priori information about the medium under investigation, the only input being a reference velocity (the speed of sound in water in this case) and the data collected in the experiment.

In our tests, we used three earth models and data which was corrupted by random noise of different magnitudes. These choices of earth models exemplify different conditions that can be found in a one-dimensional medium with variable velocity. As the level of noise was increased, we noticed that the image produced by the algorithm was deteriorating. Following standard seismic processing techniques, we applied a simple threshold filter to mute any signal with an amplitude lower than 0.05 . This filter removed all the random noise but also some of the signal used in the imaging algorithm. After running the filtered data through the imaging algorithm, we noticed that the location of the interfaces of the seismic models were still perfectly located. The amplitude was also recovered satisfactorily although the noise and the applied filters affected the final image somewhat.

These results are promising and warrant further research. Some of the planned future work includes imaging data with missing low frequencies and extending the algorithm to 1.5 and 2-dimensional media.

\section{ACKNOWLEDGMENTS}

This material is based on work supported by the National Science Foundation's REU Program IIS1004447 and grants MRI-0959461 and CNS-0922644. Any opinions, findings, and conclusions or recommendations expressed in this material are those of the author(s) and do not necessarily reflect the views of the NSF. 


\section{REFERENCES}

[1] Weglein, A.B., Araujo, F.V., Carvalho, P.M., Stolt, R.H., Matson, K.H., Coats, R.T., Corrigan, D., Foster, D.J., Shaw, S.A. and Zhang, H. (2003). Inverse scattering series and seismic exploration, Inverse Problems 19(6): R27R83.

[2] Soriano, K., Oonlamom, T. and Nita, B.G. (2013). Imaging seismic data using inverse scattering theory, International Journal of Tomography and Simulation 23(2): 19.

[3] Nita, B.G. (2009). An algorithm for seismic imaging and amplitude correction derived from scattering theory, International Journal of Tomography and Statistics 11(SO9): 118133.

[4] Tasy, A., Santamaria, G. and Nita, B.G. (2013). Numerical evaluation of a one dimensional in- verse scattering algorithm for simultaneous seismic imaging and inversion, Applied Mathematics and Computation 219(10): 50495069.

[5] Shaw, S.A. (2005). An inverse scattering series algorithm for depth imaging of reflection data from a layered acoustic medium with an unknown velocity model, Ph.D. Thesis, University of Houston, TX.

[6] Kearey, P., Brooks, M., Hill, I. (2002). An Introduction to Geophysical Exploration, Wiley-Blackwell. 3rd edition.

[7] Yilmaz, O. (2001). Seismic Data Analysis: Processing, Inversion and Interpretation of Seismic Data, Society of Exploration Geophysicists. 2nd edition.

[8] Weglein, A.B., Dragoset, W.H (2005). Multiple Attenuation, Society Of Exploration Geophysicists. [9] Stolt, R.H., Weglein A.B. (2012). Seismic Imaging and Inversion, Cambridge University Press.

[10] Gulunay, N. (2000). Noncausal spatial prediction filtering for random noise reduction on 3-D poststack data: Geophysics,65, 1641-1653.

[11] Ristau, J.P., Moon, W.M. (2001). Adaptive filtering of random noise in 2-D geophysical data: Geophysics, 66, 342-349.

[12] Fomel, S., Liu, Y. (2008). Seismic data analysis with one-dimensional seislet frame: 78th Annual International Meeting, SEG, Expanded Abstracts, 2581-2585.

[13] Lu, W., Liu, J (2007). Random noise suppression based on discrete cosine transform: 77th Annual International Meeting, SEG, Expanded Abstracts, 2668-2672.

[14] (Neelamani et al., 2008). Neelamani, R., Baumstein, A.I., Gillard, D.G., Hadidi, M.T., Soroka, W.I. (2008). Coherent and random noise attenuation using the curvelet transform: The Leading Edge, 27, 240-248.

\section{ABOUT THE STUDENT AUTHOR}

Christopher Smith graduated from The College of New Jersey in 2012 with a BS in Computer Science. He is now a Software Engineer at ZocDoc, NY. The work in this paper was completed while enrolled in the NSF funded REU program iMagine in the Computer Science Department at Montclair State University.

\section{PRESS SUMMARY}

All imaging procedures (from seismic exploration to medical) assume a velocity model for their targeted media. For example, medical ultrasound assumes that human body is made out of water and therefore sound waves that traverse it propagate with a speed of $1500 \mathrm{~m} / \mathrm{s}$. Seismic exploration methods also assume a more complex velocity model, which usually is inaccurate and produces false images of the subsurface. Inverse scattering theory provides an infrastructure that makes possible imaging without these initial assumptions. In this paper we present such an algorithm and show its ability to image noisy data. 


\title{
Effect of Native American Bean-corn Biculture Planting on Free-living Bacterial Abundance and Plant Growth
}

\author{
Heather A. Miller ${ }^{* 1}$, Justin Fiene 2 \& Tamara L. Marsh ${ }^{1}$ \\ ${ }^{1}$ Department of Biology, Elmhurst College, Elmhurst, IL \\ ${ }^{2}$ Onondaga Community College, Syracuse, NY \\ Student: heather.miller@net.elmhurst.edu \\ Mentors: marsht@elmhurst.edu,j.fiene@sunyocc.edu
}

\begin{abstract}
Maize, bean, and squash have been intercropped for thousands of years, sustaining Maya Indians and Native American tribes with bountiful harvests. Today it is widely recognized that this associated intercropping system derives much of its success from symbiotic bacteria (e.g. Rhizobium). These bacteria colonize the roots of leguminous plants, allowing them to fix atmospheric nitrogen into ammonia. However, the effect of this intercropping practice on the microbial community, independent of the effect of the symbiotic nitrogen-fixing bacteria, is not well understood. Therefore, a study was designed to model the effects of simultaneously intercropping bean and corn on the abundance of aerobic heterotrophic, free-living nitrogen-fixing, and symbiotic nitrogen-fixing bacteria, as well as plant growth and fecundity markers. In parallel, the benefits mediated by rhizobia were evaluated by inoculating a duplicate set of treatments with $\mathrm{N}$-Dure, a rhizobia-containing inoculum. Native American varieties of pole-bean (Phaseolus vulgaris L.) and corn (Zea mays mays L.) were planted in monoculture and biculture treatments. All cultivations were maintained under greenhouse conditions for 52 days with daily watering and no additional fertilizer or microbial amendments. Although a significant increase in weight per plant was noted for the inoculated biculture when compared to either the inoculated bean or corn monocultures $(p \leq 0.05)$, the abundance of heterotrophic and free-living nitrogen-fixing bacteria did not show a significant change from the related controls, with or without inoculation. However, symbiotic nitrogen-fixing bacteria, as measured by root nodulation, increased significantly $(p \leq 0.05)$ for the inoculated biculture and single planting. Thus, these data confirm that corn benefited from this associated intercropping system as shown by an increase in plant biomass that can be attributed to Rhizobium. However, neither the legume-bacteria symbiotic relationship nor the increase in plant biodiversity resulting from this intercropping practice appears to have had significant effects on the abundance of the two common soil-associated bacterial groups evaluated, though further research would be necessary to fully assess the changes to heterotrophic bacterial diversity at the species level.
\end{abstract}

\section{KEYWORDS}

Three Sisters; Nitrogen-fixing Bacteria; Inoculation with Rhizobia; Plant Growth Promoting Bacteria; Soil Microbial Biota; Corn and Bean Simultaneous Planting.

\section{INTRODUCTION}

The practice of an associated intercropping, maize and bean specifically, can be traced to the Yucatan peninsula over 6000 years ago, where the Maya Indians employed a milpa system of agriculture: maize, common or lima bean, and native squash seeds haphazardly planted together in the same hole ${ }^{1,2}$. As staple agricultural crops were being domesticated between 12,000 and 6,000 years ago throughout North America ${ }^{3}$, planting schemes involving maize, bean, and squash were being optimized. This successful planting scheme was shared with neighboring tribes, making its way to the Northeastern US between 1,000-1,200 A.D. 1,2,4, where it was known as the Three Sisters.

Scientists have since been able to shed light on why the intercropping practice was so productive. 
A key reason for the success of the triumvirate of squash, maize, and bean is the fact that their above- and below-ground physical growth characteristics are complementary and in some cases facilitate each other. ${ }^{5-7}$ Considering the above-ground characteristics, squash forms the lower canopy of this system and produces dense foliage that shades the ground, lowering the ground temperature while promoting moisture retention and weed suppression. ${ }^{7,8}$ Maize then forms the vertical canopy of this system, ${ }^{5}$ serving as a structure on which the beans can climb; thus, the growth characteristics of corn complements those of beans. ${ }^{9}$ Lastly, bean utilizes the unoccupied mid-story between squash and maize, in turn maximizing use of available sunlight for photosynthesis. ${ }^{5,10}$

Considering the below-ground characteristics, the large lateral root architecture of corn complements the vertical taproot growth of beans and squash, resulting in efficient utilization $5,11,12$ and sharing of resources. ${ }^{13,14}$ Second, arbuscular mycorrhizal fungi commonly associated with legumes have been credited with facilitating the transfer of nitrogen and other available resources to other non-leguminous plants, ${ }^{13,15}$ especially during root decomposition. ${ }^{16}$ Third, Rhizobium species that are commonly associated with legumes have been shown to inhibit the growth of soil-borne pathogens (e.g. Fusarium spp., Phytophthora root rot). ${ }^{17-19}$ Lastly, and arguably most importantly, bacteria symbiotic with leguminous plants fix atmospheric nitrogen into ammonia. ${ }^{20,21}$ This self-sustaining source of biologically available nitrogen is accessible to the bean, in turn promoting plant growth. ${ }^{22}$ The symbiotic relationship between bean and Rhizobium may be enhancing the growth of the intercropped plants in three ways: (i) by reducing the effect of beans as competitors for nitrogen in the soil, and/or, (ii) by 'leaking' excess nitrogen into the soil via root exudates that the other plants can access ${ }^{23}$ and/or, (iii) by making a useful form of atmospheric nitrogen available to surrounding non-legumes through the decomposition of bean biomass. The optimization of the conversion of atmospheric nitrogen to ammonia by Rhizobium has been the focus of novel sustainable intercropping practices, including the development and application of bacterial soil inoculants that promote plant productivity. ${ }^{24-26}$ One study revealed a correlation between the abundance of indigenous rhizobia to the efficacy of the inoculant to promote root nodulation (i.e. low initial rhizobia population resulted in an increase in the quantity and mass of nodules). ${ }^{27}$

Although foliar and root complementation can at least partially explain the growth and performance benefits yielded by the Three Sisters planting scheme, it is also possible that the increase in plant biodiversity, specifically from a monoculture to a biculture or polyculture, facilitates a beneficial change to the soil microbial community ${ }^{28-31}$ in ways that promotes microbial heterogeneity and reduction of pathogenic species. ${ }^{17-19}$ In addition, Spehn et al. (2000) reported a significant positive correlation between plant species richness and microbial biomass. They also noted that when legumes were absent, microbial biomass declined significantly regardless of plant species diversity, suggesting that the presence of beans in a biculture or polyculture planting may impact the abundance of soil microbes. In addition to symbiotic bacteria (e.g. Rhizobium spp.), associated rhizosphere bacteria (e.g. Pseudomonas spp.) have been found to significantly promote the growth of non-legumes (e.g. maize, wheat, barley, mustard) either in combination with symbiotic microorganisms or independently. ${ }^{32-34}$ This suggests that a change in the composition of the bacterial community, such as an increase or decrease in free-living nitrogen fixers or other plant growth-promoting rhizobacteria (PGPR), may potentially benefit corn directly by converting soil-based resources into biologically available forms or facilitating plant absorption of insoluble minerals.

Focusing specifically on legume-maize intercropping experiments, several reports provide evidence indicating significant benefits for maize, the legume, or both plants when they are intercropped, ${ }^{35-38}$ though contradictory evidence exists as well. ${ }^{39-41}$ It is widely accepted that rotat$\mathrm{ing}^{42}$ between or intercropping a cereal and legume crop, coupled with an adequate population of symbiotic nitrogen-fixing bacteria and/or PGPR have generally positive influences on agriculture. However, it is not clear whether these are mutually exclusive: are rhizobia solely responsible for the improved growth and performance experienced by the Three Sisters? Or have bean and/or corn facilitated a positive, synergistic effect on the general heterotrophic bacterial community that is 
known to accompany a diverse planting? Noting that squash appears to serve this intercropping system only by promoting weed suppression and moisture retention, the scope of initial testing was reduced to focus on the effects of corn on beans and vice-versa, making the experimental design more manageable. As such, the purpose of this study was to investigate whether soil microbial biota can be identified as the major mediators of enhanced plant growth. The three features of the soil microbial biota investigated were the abundance of: (i) symbiotic leguminous nitrogen-fixing bacteria (e.g. Rhizobium spp. and Bradyrhizobium spp.), as measured through nodulation of the bean root system; (ii) free-living nitrogen-fixing bacteria, (used as an indicator of a change in the plant growth promoting bacterial population) and (iii) heterotrophic bacteria (used as a general indicator of large-scale effects on the bacterial community).

Our hypotheses are that intercropping corn and bean will promote conditions, independent of inoculation, that: (a) result in the increased nodulation of the bean root system indicating an increase in the symbiotic nitrogen-fixing bacterial population; (b) result in a decrease in the abundance of free-living nitrogen-fixing bacteria, which in turn represents a general change in the composition of the free-living heterotrophic bacterial community; and, (c) facilitate enhanced, concomitant growth of bean and corn in response to these effects. ${ }^{29}$ To address these hypotheses, plant performance was assessed for corn and bean under three cultivation conditions: (1) corn and bean planted singly (i.e. one plant per pot); (2) two corn or bean plants cultivated in an individual pot (hereafter referred to as "monoculture"); and, (3) one corn and one bean plant cultivated in the same pot (hereafter referred to as "biculture"). To assess the role of symbiotic nitrogen-fixing bacteria, we inoculated a duplicate set of the aforementioned treatments with N-Dure, a commercial soil inoculum containing Rhizobium and Bradyrhizobium spp. Total heterotrophic and free-living nitrogen-fixing bacteria were quantitated for all treatments.

\section{MATERIALS AND METHODS}

Native American varieties of Phaseolus vulgaris L. (Fortex pole-bean, Hinterland Trading) and Zea mays mays cv. Mandan Bride (Organic Mandan Bride corn, Hirt's Gardens) were grown under temperate greenhouse conditions during the months of July and August 2013 in Elmhurst, IL. Plants were grown in a previously unused fertilizer enriched potting soil (NPK 12:9:7; Schultz', Infinity Lawn and Garden, Inc.; nutrient content was assumed as advertised by the manufacturer) for 52 days, with water supplied on average every 24 hours and without additional fertilizer.

\section{SEED SELECTION AND PREPARATION}

Based on a 30-day preliminary study demonstrating size-dependent germination success (data not shown), bean seeds weighing $0.43 \mathrm{~g}-0.55 \mathrm{~g}$ were selected; this correlation was not demonstrated by the corn seeds, therefore these seeds were randomly selected. All seeds were rinsed in sterile water prior to being forcibly germinated. Germination was forced by arranging the seeds between damp paper towels inside new polyethylene zipper storage bags, and storing those bags in a dark growth chamber maintained at $30^{\circ} \mathrm{C}$. Upon visual inspection, only seeds that produced a wellformed radicle were selected for planting.

\section{EXPERIMENTAL DESIGN}

To investigate the effect of associated intercropping on corn and bean growth, five treatments were imposed $(\mathrm{N}=3)$ : Corn and bean were either planted alone (referred to as $\mathrm{C}$ or $\mathrm{B}$ ), in monocultures comprised of two corn (CC) or two bean (BB) plants in the same pot, or in a biculture comprised of one corn and one bean in the same pot (CB). To identify the effect of rhizobia on plant performance and on the abundance of key microbial communities in each condition, an identical set of treatments was established that were inoculated with N-Dure, an alfalfa/true clover inoculant (INTX Microbials, LLC) containing Bradyrhizobium japonicum and Rhizobium species. Both 
corn and bean radicle-producing seeds were gently rolled in the inoculant prior to planting using sterile tweezers, and the surface of the soil was amended with approximately $1 \mathrm{~g}$ of inoculant post planting. Unplanted soil controls with and without N-Dure were maintained under the previously mentioned conditions. All forcibly germinated seeds were gently placed in the soil to a depth of 2-4 $\mathrm{cm}$, radicle down, and approximately $5 \mathrm{~cm}$ apart in the pot if planted in monoculture or biculture treatments.

\section{PLANT MEASUREMENTS}

The plants were grown for 52 days, at which time the experiment was terminated and various measures of plant and bacterial growth were recorded. For corn, the following data points were collected: stalk height as determined from just above root system to tallest point of the top leaf, number of leaves with visible leaf collars excluding visibly deteriorated lower leaves, presence of exposed tassel, and aboveground biomass. For bean, the following data points were collected: number of trifoliate leaves excluding visibly deteriorated lower leaves, number of flowers and bean pods, and aboveground biomass. The root systems of all bean plants were destructively sampled for root nodules. To ensure comparable results across replicates, a minimum nodule size (diameter $\geq 1 \mathrm{~mm}$ ) and time limit threshold (90 minutes) was established, and nodules subsequently enumerated.

\section{BACTERIAL MEASUREMENTS}

Prior to using the newly procured potting soil, a three-point most probable number (MPN) dilution technique carried out to a $10^{11}$ dilution ${ }^{43}$ was executed at $\mathrm{T}_{0}$ for both general heterotrophs and free-living nitrogen-fixing bacteria. After collecting the aboveground measurements, the numbers of culturable aerobic heterotrophic and free-living nitrogen-fixing bacteria were enumerated using the same MPN technique. The $10^{0}$ soil inoculation sample $(0.5 \mathrm{~g})$ was collected from the center of each pot approximately $1-2 \mathrm{~cm}$ below the surface, or between mono- or biculture treatments where applicable. Aerobic heterotrophs were enumerated using a half-strength nutrient broth (Difco). Free-living nitrogen-fixing bacteria were enumerated using a modified Ashby's nitrogen-free broth containing the following per liter: mannitol $20 \mathrm{~g} ; \mathrm{K}_{2} \mathrm{PO}_{4} 0.2 \mathrm{~g} ; \mathrm{MgSO}_{4} \cdot 7 \mathrm{H}_{2} \mathrm{O} 0.25 \mathrm{~g}$; $\mathrm{NaCl} 0.2 \mathrm{~g}$ (Ashby, 1907); Tanner's trace metals 5ml; and $\mathrm{CaCO}_{3} 3 \mathrm{~g} \cdot{ }^{44}$ Individual broth tubes were scored positive if visible growth was observed after an incubation period of 48 hours at $23^{\circ} \mathrm{C}$ for aerobic heterotrophs, or after an incubation period of 7 days at $23^{\circ} \mathrm{C}$ for free-living nitrogen fixers.

\section{STATISTICAL ANALYSIS}

A series of Student's t-tests were performed to evaluate the effects of the associated intercropping system and the inoculation treatments on the plant and microbial measurements. In addition, an evaluation of the correlation between two quantitative variables (e.g., nodules per bean plant versus weight per plant) and the inoculation treatment or associated intercropping system was performed using analysis of variance (ANOVA), noting data were normally distributed (Shapiro-Wilk; $p>0.05)$. Where the ANOVA indicated significant effects or interactions, a Tukey's HSD post-hoc multiple means comparison test was used to identify the specific differences in among treatment conditions. Significance of difference was evaluated at $p \leq 0.05$ for all statistical analyses. Data analyses were performed in Microsoft Excel $^{\oplus}$ (version 14.3.9, 2011) and in $\mathrm{R}^{\odot}$, freeware made available by The R Foundation for Statistical Computing (version 3.0.1, 2013).

\section{RESULTS}

Although the productivity of various intercropping designs has been studied at length, ${ }^{22,35-41}$ the focus of this research was placed on the microflora. We sought to evaluate the microbial response and subsequent impact on plant performance of a corn-bean biculture planting scheme. In this study we attempted to identify the effects of intercropping Native American varieties of bean and 
corn on the growth and fecundity of each individual of the biculture or monoculture. These results were then compared to the abundance of bacteria enumerated for each replicate, inoculated and not inoculated with rhizobia, with the goal of revealing correlations between plant performance and a change in the composition of the microbial community.

\section{PLANT BIOMASS AND CHARACTERISTICS}

Bean-Corn Biculture

The corn plants present in the inoculated biculture demonstrated a statistically significant increase in weight per plant when compared to the inoculated corn monoculture (Figure $1 ; p=0.025$ ); this represented the second largest mean biomass weight recorded for corn, following singly planted corn. These data also revealed a statistically significant $44 \%$ increase in corn biomass weight when comparing the inoculated to the un-inoculated biculture planting treatments (Table 1, $p=0.034$ ). The bean plant present in the inoculated biculture planting treatment however, was not statistically different from the inoculated bean monoculture (Figure 2; $p=0.30$ ). In addition, a $51 \%$ increase in bean biomass weight was noted when comparing the inoculated to the un-inoculated biculture planting treatments, but again this was not statistically significant (Table 2, $p=0.117$ ). Out of all un-inoculated treatments containing bean or corn, lowest biomass values were recorded for the biculture, though this was not significantly different from either the bean or corn monoculture treatments, respectively.

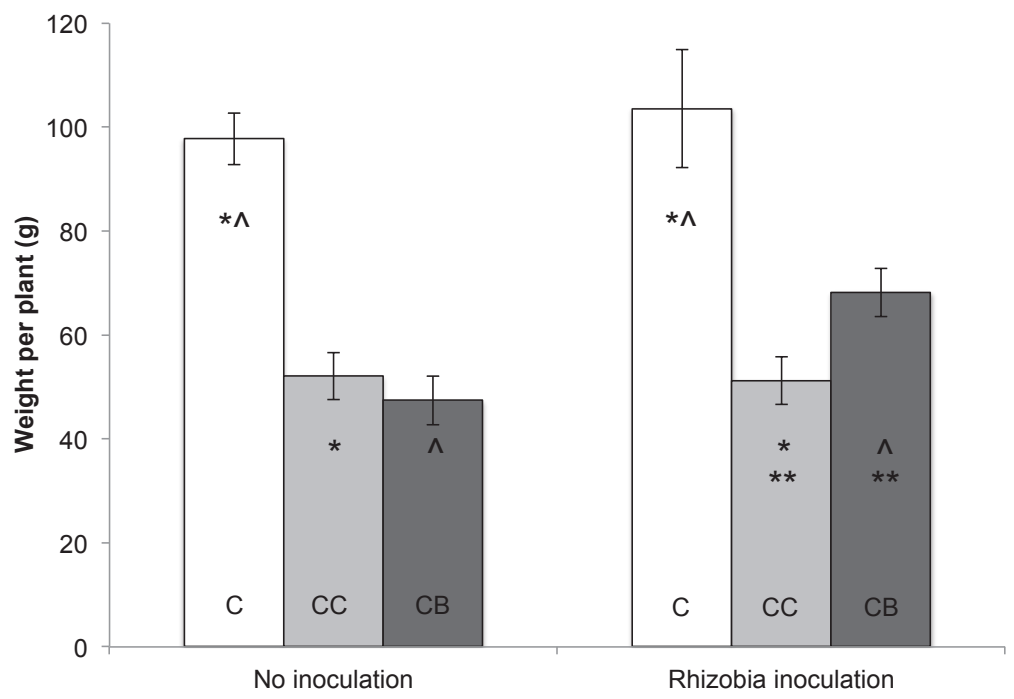

Figure 1. Comparison of mean aboveground biomass weight per corn plant for each inoculation treatment across each planting scheme. Empty bars represent single corn plantings (C), light-grey bars represent monoculture corn plantings (CC), and dark-grey bars represent corn-bean biculture plantings (CB). Bars are mean values $\pm S E, N=3$. Significant differences are denoted with an ${ }^{\star}, \wedge$, and ${ }^{* *}(p \leq 0.05$, Student's t-test) 


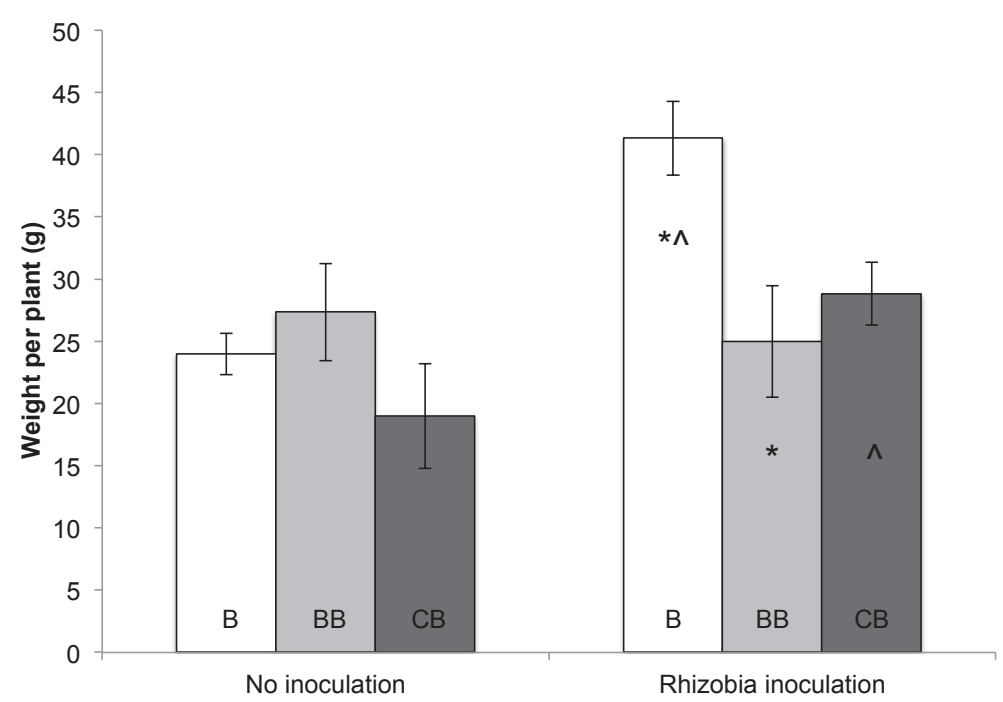

Figure 2. Comparison of mean aboveground biomass weight per bean plant for each inoculation treatment across each planting scheme. Empty bars represent single bean plantings (B), light-grey bars represent monoculture bean plantings (BB), and dark-grey bars represent corn-bean biculture plantings (CB). Bars are mean values $\pm \mathrm{SE}, \mathrm{N}=3$. Significant differences are denoted with an ${ }^{*}$ and $\wedge(p \leq 0.05$, Student's t-test $)$

The inoculation had a significant positive effect on the number of root nodules found on the biculture bean, representing a $690 \%$ increase from the un-inoculated biculture bean (Figure 3; $p=0.043$ ). While, nodulation of the bean root system for the un-inoculated planting treatments was found to be similar between the biculture and the singly planted bean $(p=0.754)$.

No significant differences were noted with the additional plant characteristics recorded for the biculture bean (number of trifoliate leaves, number of flowers, number of seed pods, nodule count; Table 2) or the biculture corn (number of collared leaves, presence of tassel; Table 1).

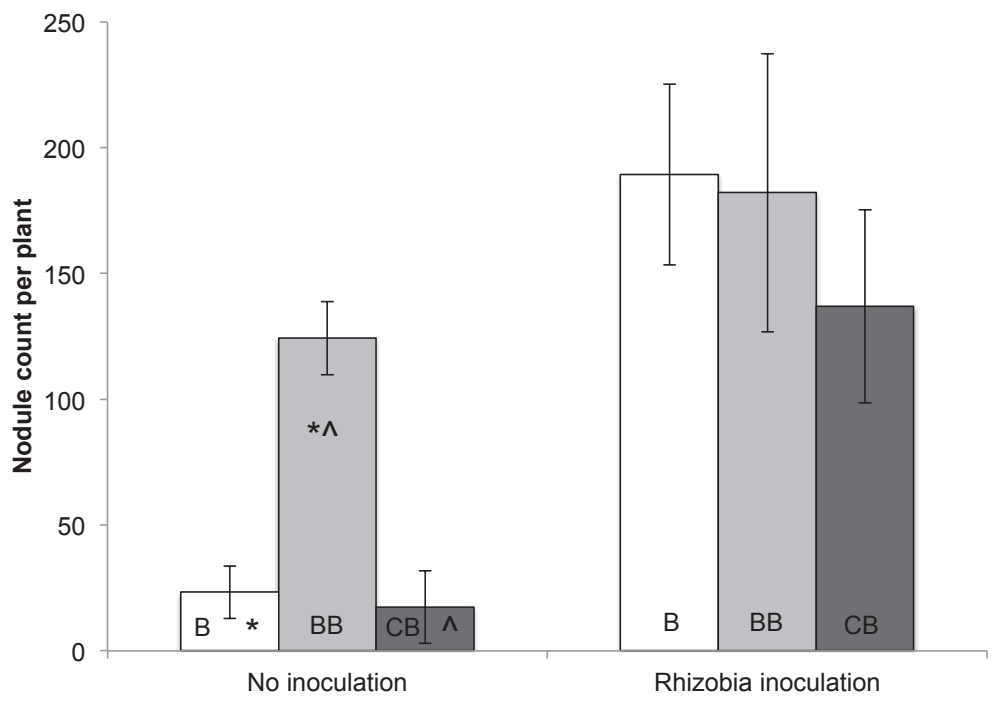

Figure 3. Comparison of mean nodule count per bean plant for each inoculation treatment across each planting scheme. Empty bars represent single bean plantings (B), light-grey bars represent monoculture bean plantings (BB), and darkgrey bars represent corn-bean biculture plantings $(\mathrm{CB})$. Bars are mean values $\pm \mathrm{SE}, \mathrm{N}=3$. Significant differences are denoted with an ${ }^{\star}$ and $\wedge(p \leq 0.05$, Student's t-test $)$ 


\section{Bean Single and Monoculture}

In the un-inoculated treatment, bean root nodules were significantly higher in monocultures when compared to the single and biculture planting treatments ( $p=0.005, p=0.007$, respectively), suggesting a facilitative effect of conspecifics on nodule growth.$^{45}$ The inoculation had a positive effect on the number of root nodules found on the single and monoculture bean, ( $711 \%$ and $47 \%$ increase, respectively), though this was only statistically significant for the singly planted bean $(p=0.011)$. Furthermore, nodule count per plant was significantly, positively correlated with bean biomass weight $(p=0.001)$, which agrees with the known symbiotic relationship between legumes and rhizobia. ${ }^{46,47}$

The inoculated single bean planting treatments demonstrated higher biomass values when compared to the inoculated bean monocultures ( $p=0.010$; Figure 2). Furthermore, highest bean biomass values were achieved under the single planting treatment that was inoculated, and were nearly double the biomass weight as those found in monocultures and bicultures. However, there were no significant differences noted in bean growth between the inoculated and un-inoculated monocultures, which generally agrees with the evidence showing that root nodule growth in monocultures were similar between inoculation treatments, and root nodules were positively correlated with growth.

No significant differences were noted with the additional bean plant characteristics recorded for either the single or monoculture bean (number of trifoliate leaves, number of flowers, number of seed pods, nodule count; Table 2).

\section{Corn Single and Monoculture}

Corn growth in the absence of bean was unaffected by the inoculation. The singly planted corn, regardless of the inoculation condition, demonstrated significantly higher biomass values when compared to the respective bicultures (inoculated, $p=0.045$; un-inoculated, $p=0.002$ ) and the corn monocultures (inoculated, $p=0.010$; un-inoculated, $p=0.005$; Figure 1). Highest corn biomass values were achieved under the single planting treatments, and were nearly twice the biomass weight as those in monocultures and bicultures. This difference can be attributed to the height of the stalk, which was statistically significant between the single and monoculture corn conditions $(p=0.030)$. No other significant differences were noted with the additional corn plant characteristics recorded (number of collared leaves, presence of tassel; Table 1). 
from the $\mathrm{T}_{0}$ controls. In addition, though not statistically significant, the abundance of free-living nitrogen fixers increased in the un-inoculated biculture when compared to the inoculum positive control pots ( $p=0.064$, Figure 4).

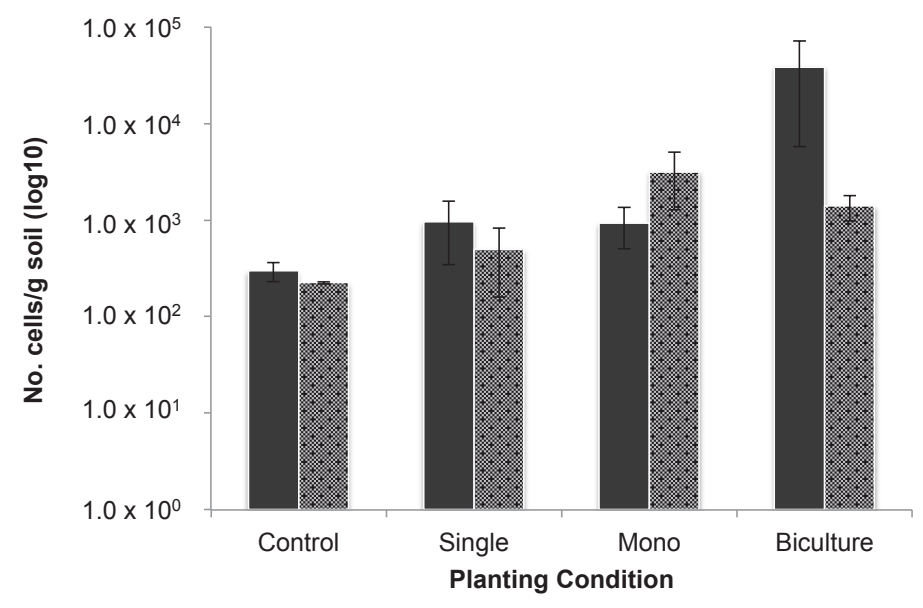

Figure 4. Free-living nitrogen fixing bacteria abundance using MPN enumeration technique. Results are presented for the combined bacterial abundances of corn and bean; i.e. single and monoculture MPN bars represent single bean + single corn and mono-bean + mono-corn, respectively. This was done for ease of reporting; no significant differences were noted for plant-based MPN. Mean abundance of freeliving nitrogen-fixing bacteria for each planting scheme were compared across inoculation treatments. Dark-grey bars represent un-inoculated plantings, and light-grey-dotted bars represent inoculated plantings. Bars are mean values \pm SE, $\mathrm{N}=3$ ( $p \leq 0.05$, Students' t-test and ANOVA)

\begin{tabular}{|c|c|c|c|c|}
\hline & \multicolumn{2}{|c|}{ General Heterotrophs (MPN) } & \multicolumn{2}{|c|}{ Free-living Nitrogen fixers (MPN) } \\
\hline \multicolumn{5}{|c|}{ Un-inoculated } \\
\hline Control & $8.05 \times 10^{10}$ & $\pm 2.95 \times 10^{10}$ & $2.96 \times 10^{2}$ & $\pm 6.53 \times 10^{1}$ \\
\hline Single Bean & $3.90 \times 10^{10}$ & $\pm 3.55 \times 10^{10}$ & $1.62 \times 10^{3}$ & $\pm 6.93 \times 10^{2}$ \\
\hline Single Corn & $8.05 \times 10^{10}$ & $\pm 2.95 \times 10^{10}$ & $2.90 \times 10^{2}$ & $\pm 6.89 \times 10^{1}$ \\
\hline Double Bean & $1.10 \times 10^{11}$ & \pm 0 & $6.31 \times 10^{2}$ & $\pm 2.17 \times 10^{2}$ \\
\hline Double Corn & $1.10 \times 10^{11}$ & $\pm 3.67 \times 10^{7}$ & $1.22 \times 10^{3}$ & $\pm 5.63 \times 10^{2}$ \\
\hline Bean + Corn & $1.10 \times 10^{11}$ & \pm 0 & $3.86 \times 10^{4}$ & $\pm 3.67 \times 10^{4}$ \\
\hline \multicolumn{5}{|l|}{ Inoculated } \\
\hline Control & $5.10 \times 10^{10}$ & $\pm 2.95 \times 10^{10}$ & $2.24 \times 10^{2}$ & $\pm 6.67 \times 10^{0}$ \\
\hline Single Bean & $1.10 \times 10^{11}$ & \pm 0 & $3.91 \times 10^{2}$ & $\pm 2.78 \times 10^{2}$ \\
\hline Single Corn & $1.10 \times 10^{11}$ & \pm 0 & $5.98 \times 10^{2}$ & $\pm 4.38 \times 10^{2}$ \\
\hline Double Bean & $1.10 \times 10^{11}$ & \pm 0 & $2.42 \times 10^{3}$ & $\pm 9.81 \times 10^{2}$ \\
\hline Double Corn & $8.05 \times 10^{10}$ & $\pm 2.95 \times 10^{10}$ & $3.91 \times 10^{3}$ & $\pm 2.71 \times 10^{3}$ \\
\hline Bean + Corn & $3.79 \times 10^{9}$ & $\pm 3.60 \times 10^{9}$ & $1.39 \times 10^{3}$ & $\pm 4.59 \times 10^{2}$ \\
\hline
\end{tabular}

Table 3. Effect of planting and inoculation treatment on microbial abundance. Mean values of the three point MPN are presented supplemented by the standard error 


\section{DISCUSSION}

This study sought to investigate the impacts of a Native American inspired intercropping system on the microbial community, and to link the changes in the soil microbial biota abundance and the overall general heterotrophic community composition to the improved performance experienced by the individual plants. The Three Sisters planting scheme was purposefully deconstructed to focus on the interactions between the plants having the greatest reported effect on microbial communities, that being corn and bean. The microbe-facilitated interactions were assessed through the quantification of symbiotic leguminous nitrogen-fixing bacteria and free-living nitrogen-fixing bacteria, as evaluated through bean root nodulation and an MPN assay, respectively. The changes observed in the microbial community were then compared to growth and fecundity characteristics of both corn and bean, by planting treatment and inoculation treatment. The results of this study indicate that symbiotic nitrogen-fixing bacteria (i.e. rhizobia) are mediating most growth benefits realized by intercropped corn, while the bean neither gains nor loses in this biculture when compared to the monoculture system.

The first hypothesis sought to identify the effect of simultaneously intercropping corn and bean on the symbiotic leguminous bacterial population as realized through an increase in the number of nodules found on the biculture bean root system, focusing primarily on the un-inoculated planting treatment. If the benefits of intercropping are independent of inoculation, then perhaps bean is driven to fix greater amounts of atmospheric nitrogen to support its metabolic needs, as well as those of the associated crop. Unfortunately, plant performance was not significantly different between either plant in the un-inoculated biculture compared to the monoculture (same conditions). Therefore, the hypothesis could not be supported by the data gathered. This was unexpected noting that Dawo et al. (2007) witnessed highest biomass yields when Phaseolus vulgaris and Zea mays were simultaneously intercropped and not inoculated with symbiotic leguminous bacteria. The replication and sampling plan was originally designed to support microbiological practices, yet may have generated insufficient data on which to accept the hypothesis, noting that Dawo et al. planted a $432 \mathrm{~m}^{2}$ field at densities ranging from $50,000-100,000$ plants ha ${ }^{-1}$. However, additional research has revealed differing results for similar un-inoculated, intercropped maize-legume systems. For example, Martin et al. (1990) reported highest corn yields from outdoor plots when it was monocropped vs. simultaneously intercropped with soybean. Risch and Hansen (1982) reported similar results from outdoor plots with a bean-corn-squash polyculture, noting that the per plant yields of both corn and squash decreased significantly, though bean yield benefited significantly. Although no benefits were realized by the un-inoculated bicultures, significant plant growth was observed for the biculture corn when coupled with significant nodulation of the bean root system, but only when this planting treatment was inoculated. This was not completely unexpected, recognizing previous research had reported benefits realized by corn when the maize-legume bicultures were collectively inoculated with symbiotic leguminous bacteria. ${ }^{22,25,26}$ The results of this research suggest that the inoculated biculture corn indirectly benefited from either the increased nodulation of the bean as a result of an increased abundance of symbiotic nitrogen-fixing bacteria or through a change in the composition of plant-growth promoting rhizobia. Noting that the inoculated biculture bean performed equally as well when planted in monocultures, and the biculture bean appeared to recover from the reduced growth experienced under the un-inoculated condition (i.e. the inoculated biculture bean witnessed a $52 \%$ increase in biomass weight from the un-inoculated biculture bean, though this was not statistically significant), this further suggests that symbiotic nitrogen-fixing bacteria may also be ameliorating the competitive effects of corn on bean when these plants are intercropped. The results witnessed during this experiment differ from those reported by Searle et al. (1981), where no effect on corn grain yield was noted and legume biomass decreased when these plants were intercropped and inoculated.

The second hypothesis sought to link a change in the abundance of free-living nitrogen-fixing bacteria regardless of inoculation treatment to benefits realized in the biculture, and concomi- 
tantly, both corn and bean growth would be facilitated. ${ }^{29}$ However, no link could be established between a change in free-living nitrogen-fixing bacterial abundance and the aboveground plant growth and performance. Instead, the abundance of free-living nitrogen-fixing bacteria increased in the un-inoculated biculture treatment when compared to the related unplanted control pots, however this was not statistically significant. These results were unexpected noting that Keswani (1976) reported an increase in rhizosphere microbial populations when maize and soybean were intercropped and un-inoculated, while also noting a significant increase in maize yield compared to a monoculture. Therefore, the bacterial abundance assessment performed may have been an ineffective measure of the full contribution mediated by free-living soil bacteria under a biculture planting scheme. For example, Pseudomonas denitrificans, P. rathonis, Bacillus laevolacticus, B. amyloliquefaciens, and Arthrobacter simplex, all aerobic or facultative anaerobic heterotrophic soil species, have been found to promote plant growth of maize, ${ }^{49}$ along with Azotobacter spp., an aerobic free-living nitrogen-fixing soil bacterium..$^{50}$ Noting no evidence of a significant change in abundance of free-living nitrogen-fixing bacteria, an evaluation of the change in species richness of general heterotrophic bacteria may have offered the expected correlation.

The third hypothesis considers the potential impact on both corn and bean growth when it is intercropped, as witnessed through the combined effects of a shift in free-living heterotrophic bacterial community composition and an increase in symbiotic leguminous bacteria as realized through increased nodulation of the bean root system. This can be best assessed through the results obtained for the inoculated biculture corn. Although not statistically significant, the decrease in abundance of free-living nitrogen-fixing bacteria for the inoculated biculture, coupled with the increased nodulation of the inoculated biculture bean suggests the inoculated biculture corn benefited from the facilitation provided by this intercropping system. This can be supported through the finding that the biculture corn realized significant benefits when inoculated. This appears to indicate that the inoculated biculture bean produced excess fixed nitrogen, making this resource available to corn, the transference of which is commonly mediated by mycorrhizal fungi. ${ }^{13,15,16,24}$ Isotopic analysis providing evidence of the transference of nitrogen from legumes to maize has revealed a complex system of resource sharing between these intercropped plants ${ }^{13}$ and it is clear that legumes inoculated with Rhizobium and intercropped with a cereal have been found to generate significantly higher amounts of ammonia both under field and greenhouse growing conditions. ${ }^{24}$ Specifically, Patra et al. (1986) reported that inoculated maize-intercropped legumes fixed approximately $32 \%$ of the total nitrogen utilized by maize.

Weight per plant across both inoculation treatments consistently demonstrated highest means for the single planting scheme. This was a reasonable expectation recognizing neither the single bean nor the single corn had to compete with other plants for soil-based resources. In effect, these treatments received access to twice the resources made available to either the monoculture or the biculture. The spatial arrangement and density of the legume-maize intercropping practice has been reported to significantly impact the yields of both plants, recognizing the competition for available growth limiting nutrients directly influences the success of this planting scheme. ${ }^{51-53}$ In evaluating the single bean results, it appears the inoculation facilitated a significant increase in bean growth, suggesting that nitrogen was a limiting resource that was mitigated by an increase in nitrogen-fixation activities performed by the bean root nodules. However, when this trend was not similarly demonstrated by the monoculture, either through an increase in biomass weight or root nodulation on a per plant basis, this suggests that physical space between plants ${ }^{51,52}$ may be just as important as the inoculation.

Although this research did not reveal a significant increase or decrease in the abundance of free-living nitrogen-fixing bacteria, which suggests little change in the overall composition of the general heterotrophic community, symbiotic nitrogen-fixing bacteria appeared to be responsible for mediating the benefits realized by bean and corn. The debate lies in whether rhizobia alone are directly or indirectly responsible. Research has found that bacterial diseases are inhibited ${ }^{54}$ and the 
soil bacterial composition is significantly impacted when legumes are intercropped with maize or other cereal crops without inoculation. ${ }^{55,56}$ Furthermore, Rhizobium inoculation has been linked to a decrease in bacterial diversity. ${ }^{27}$

Regardless of the inoculation treatment, when these two 'sisters' are planted simultaneously and in close proximity, the bean-corn intercropping system reaps rewards greater than or equal to a monocrop system. The physical growth synergies realized both above and below ground by corn and bean, coupled with mutualistic microbial interactions represent a complex set of factors that govern this intercropping system. In an effort to fully vet the contributions made directly or facilitated indirectly by the bacterial community will require further research. First, squash should be incorporated into the planting treatments to allow for a more complete evaluation of bacterial abundance changes as a result of intercropping all three 'sisters'. Second, alternative methods may need to be employed. It is possible the microbial community associated with a Three Sisters planting scheme changes over time, which would suggest the use of soil obtained from a garden where these plants have been intercropped for successive growing seasons. Third, it is possible that the sample sizes appropriate for microbiological assays may have been insufficient to achieve the levels of significance necessary when conducting plant assays. As a result, plant replicates and sampling would need to be increased in order to fully vet the currently suggestive trends in the microbiologically mediated performance of corn and bean. Lastly, even though the MPN did not reveal significant changes in the abundance of the microbial communities we evaluated, it is possible that the abundance of specific general heterotrophic species are impacted by this intercropping system, in turn positively affecting the composition of the entire soil bacterial community. Valuable insights could be ascertained through the use of molecular techniques, allowing for detection and discernment of important shifts in species that may contribute to the success of this intercropping system.

\section{ACKNOWLEDGEMENTS}

We sincerely thank Andrea Poplawski and Laura Merkle for their unwavering commitment to this project as recognized through the $>3000$ culture tubes into which they dispensed media and serially diluted soil samples supporting the bacterial abundance MPN study. We also thank the students of Microbial Ecology (Fall 2013) for taking care to examine and remove nodules from the root balls of all bean plant replicates. Without them, these data may have been lost to decomposition or dehydration of the nodules.

\section{REFERENCES}

[1] Emerson, R.A. (1953) A preliminary survey of the milpa system of maize culture as practiced by the Maya Indians of the northern part of the Yucatan Peninsula, Ann Mo Bot Gard. 40, 51-62.

[2] Lewandowski, S. (1987) Diohe'ko, the Three Sisters in Seneca life: Implications for a native agriculture in the finger lakes region of New York State, Agric Human Values 4, 76-93.

[3] Smith, B.D. (2006) Eastern North America as an independent center of plant domestication, Proc Natl Acad Sci U.S.A. 103, 12223-12228.

[4] Smith, B.D. (2006) Rivers of Change, 3rd ed. University of Alabama Press, Tuscaloosa.

[5] Postma, J.A., Lynch, J.P. (2012) Complementarity in root architecture for nutrient uptake in ancient maize/bean and maize/bean/squash polycultures, Annals of botany 110, 521-534.

[6] Tilman, D., Reich, P.B., Knops, J., Wedin, D., Mielke, T., Lehman, C. (2001) Diversity and productivity in a long-term grassland experiment, Science 294, 843-845.

[7] Liebman, M., Dyck, E. (1993) Crop rotation and intercropping strategies for weed management, Ecol Appl 3, 92-122.

\section{$\mathrm{AJUR}$ Volume $12 \mid$ Issue 4 | November 2015}


[8] Fujiyoshi, P.T., Gliessman, S.R., Langenheim, J.H. (2007) Factors in the suppression of weeds by squash interplanted in corn, Weed Biol Manag 7, 105-114.

[9] Scarry, C.M. (2008) Crop husbandry practices in North America's eastern woodlands, in Case Studies in Environmental Archaeology (Reitz E., Scudder, S., Scarry, C.M. Ed.). pp. 391-404, Springer New York.

[10] Bilalis, D., Papastylianou, P., Konstantas, A., Patsiali, S., Karkanis, A., Efthimiadou, A. (2010) Weed-suppressive effects of maize-legume intercropping in organic farming, Int J of Pest Manag 56, 173-181.

[11] Pagès, L., Pellerin, S. (1994) Evaluation of parameters describing the root system architecture of field grown maize plants (Zea mays L.), Plant Soil 164, 169-176.

[12] Weaver, J.E., Bruner, W.E. (1927) Root development of vegetable crops. McGraw-Hill Book Company.

[13] Carlsson, G., Huss-Danell, K. (2013) Does nitrogen transfer between plants confound 15N-based quantifications of $\mathrm{N} 2$ fixation?, Plant Soil.

[14] Giller, K.E., Ormesher, J., Awah, F.M. (1991) Nitrogen transfer from Phaseolus bean to intercropped maize measured using $15 \mathrm{~N}$-enrichment and 15N-isotope dilution methods, Soil Biol Biochem 23, 339346.

[15] Frey, B., Schüepp, H. (1993) A role of vesicular-arbuscular (VA) mycorrhizal fungi in facilitating interplant nitrogen transfer, Soil Biol Biochem 25, 651-658.

[16] Johansen, A., Jensen, E.S. (1996) Transfer of $N$ and $P$ from intact or decomposing roots of pea to barley interconnected by an arbuscular mycorrhizal fungus, Soil Biol Biochem 28, 73-81.

[17] Buonassisi, A.J., Copeman, R.J., Pepin, H.S., Eaton, G.W. (1986) Effect of Rhizobium spp. on Fusarium solani f. sp. phaseoli, Can J Plant Pathol 8, 140-146.

[18] Tu, J.C. (1978) Protection of soybean from severe Phytophthora root rot by Rhizobium, Physiol Plant Pathol 12, 233-240.

[19] Omar, S.A., Abd-Alla, M.H. (1998) Biocontrol of fungal root rot diseases of crop plants by the use of rhizobia and bradyrhizobia, Folia Microbiol 43, 431-437.

[20] Dakora, F.D., Keya, S.O. (1997) Contribution of legume nitrogen fixation to sustainable agriculture in sub-saharan Africa, Soil Biol Biochem 29, 809-817.

[21] Zahran, H.H. (1999) Rhizobium-legume symbiosis and nitrogen fixation under severe conditions and in an arid climate, Microbiol Mol Biol Rev 63, 968-989.

[22] Peoples, M.B., Brockwell, J., Herridge, D.F., Rochester, I.J., Alves, B.J.R., Urquiaga, S., Boddey, R.M., Dakora, F.D., Bhattarai, S., Maskey, S.L., Sampet, C., Rerkasem, B., Khan, D.F., Hauggaard-Nielsen, H., Jensen, E.S. (2009) The contributions of nitrogen-fixing crop legumes to productivity of agricultural systems, Symbiosis 48, 1-17.

[23] Ta, T.C., Macdowall, F.D.H., Faris, M.A. (1986) Excretion of nitrogen assimilated from N2 fixed by nodulated roots of alfalfa (Medicago sativa), Canadian Journal of Botany 64, 2063-2067.

[24] Patra, D.D., Sachdev, M.S., Subbiah, B.V. (1986) 15N studies on the transfer of legume-fixed nitrogen to associated cereals in intercropping systems, Biol Fertil Soils 2, 165-171.

[25] Pineda, P., Kipe-Nolt, J.A., Rojas, E. (1994) Rhizobium inoculation increases of bean and maize yields in intercrops on farms in the Peruvian Sierra, Exp Agric 30, 311-318. 
[26] Thies, J.E., Singleton, P.W., Bohlool, B.B. (1991) Influence of the size of indigenous rhizobial populations on establishment and symbiotic performance of introduced rhizobia on field-grown legumes, Appl Environ Microbio 57, 19-28.

[27] Zhang, N., Sun, Y., Li, L., Wang, E., Chen, W., Yuan, H. (2010) Effects of intercropping and Rhizobium inoculation on yield and rhizosphere bacterial community of faba bean (Vicia faba L.), Biol Fertil Soils 46, 625-639.

[28] Kuske, C.R., Ticknor, L.O., Miller, M.E., Dunbar, J.M., Davis, J.A., Barns, S.M., Belnap, J. (2002) Comparison of soil bacterial communities in rhizospheres of three plant species and the interspaces in an arid grassland, Appl Environ Microbio 68, 1854-1863.

[29] Spehn, E.M., Joshi, J., Schmid, B., Alphei, J., Körner, C. (2000) Plant diversity effects on soil heterotrophic activity in experimental grassland ecosystems, Plant Soil 224, 217-230.

[30] Westover, K.M., Kennedy, A.C., Kelley, S.E. (1997) Patterns of rhizosphere microbial community structure associated with co-occurring plant species, J Ecol 85, 863-873.

[31] Zak, D.R., Holmes, W.E., White, D.C., Peacock, A.D., Tilman, D. (2003) Plant diversity, soil microbial communities, and ecosystem function: are there any links?, Ecology 84, 2042-2050.

[32] Chabot, R., Antoun, H., Cescas, M. (1996) Growth promotion of maize and lettuce by phosphate-solubilizing Rhizobium leguminosarum biovar. phaseoli, Plant Soil 184, 311-321.

[33] Hayat, R., Ali, S., Amara, U., Khalid, R., Ahmed, I. (2010) Soil beneficial bacteria and their role in plant growth promotion: a review, Ann Microbiol 60, 579-598.

[34] Höflich, G., Wiehe, W., Kühn, G. (1994) Plant growth stimulation by inoculation with symbiotic and associative rhizosphere microorganisms, Experientia 50, 897-905.

[35] Ahmed, S., Rao, M.R. (1982) Performance of maize-soybean intercrop combination in tropics: results of a multi-location study, Field Crops Res. 5, 147-161.

[36] Dawo, M.I., Wilkinson, J.M., Sanders, F.E.T., Pilbeam, D.J. (2007) The yield and quality of fresh and ensiled plant material from intercropped maize (Zea mays) and beans (Phaseolus vulgaris), J Sci Food Agric 87, 1391-1399.

[37] Ngwira, A.R., Aune, J.B., Mkwinda, S. (2012) On-farm evaluation of yield and economic benefit of short term maize legume intercropping systems under conservation agriculture in Malawi, Field Crops Res. 132, 149-157.

[38] Willey, R.W., Osiru, D.S.O. (1972) Studies on mixtures of maize and beans (Phaseolus vulgaris) with particular reference to plant population, J Agric Sci 79, 517-529.

[39] Martin, R.C., Voldeng, H.D., Smith, D.L. (1990) Intercropping corn and soybean for silage in cool-temperature region: yield, protein and economic effects, Field Crops Res. 23, 295-310.

[40] Risch, S.J., Hansen, M.K. (1982) Plant growth, flowering phenologies, and yields of corn, beans and squash grown in pure stands and mixtures in Costa Rica, J Appl Ecol 19, 901-916.

[41] Searle, P.G.E., Comudom, Y., Shedden, D.C., Nance, R.A. (1981) Effect of maize+legume intercropping systems and fertilizer nitrogen on crop yields and residual nitrogen, Field Crops Res. 4, 133-145.

[42] Kirkegaard, J., Christen, O., Krupinsky, J., Layzell, D. (2008) Break crop benefits in temperate wheat production, Field Crops Res. 107, 185-195.

[43] Banwart, G.J. (1981) Basic food microbiology. AVI Pub. Co., Westport, Conn. 
[44] Tanner, R.S. (1989) Monitoring sulfate-reducing bacteria: comparison of enumeration media, J Microbiol Methods 10, 83-90.

[45] Nambiar, P.T.C., Rao, M.R., Reddy, M.S., Floyd, C.N., Dart, P.J., Willey, R.W. (1983) Effect of intercropping on nodulation and N2-fixation by groundnut, Exp Agric 19, 79-86.

[46] Hungria, M., Campo, R.J., Mendes, I.C. (2003) Benefits of inoculation of the common bean (Phaseolus vulgaris) crop with efficient and competitive Rhizobium tropici strains, Biol Fertil Soils 39, 88-93.

[47] Weaver, R.W., Frederick, L.R. (1974) Effect of inoculum rate on competitive nodulation of Glycine max L. Merrill. II. Field Studies1, Agron J, 233-236.

[48] Keswani, C.L., Kibani, T.H.M., Chowdhury, M.S. (1977) Effect of intercropping on rhizosphere population in maize (Zea mays L.) \& soybean (Glycine max Merrill), Agric Environ 3, 363-368.

[49] Egamberdiyeva, D. (2005) Plant-growth-promoting rhizobacteria isolated from a Calcisol in a semi-arid region of Uzbekistan: biochemical characterization and effectiveness, J Plant Nutr Soil Sci 168, 94-99.

[50] Zahir, Z.A., Abbas, S.A., Khalid, M., Arshad, M. (2000) Substrate dependent microbially derived plant hormones for improving growth of maize seedlings, Pak J Biol Sci 3, 289-291.

[51] Chui, J.A.N., Shibles, R. (1984) Influence of spatial arrangements of maize on performance of an associated soybean intercrop, Field Crops Res. 8, 187-198.

[52] Davis, J.H.C., Roman, A., Garcia, S. (1987) The effects of plant arrangement and density on intercropped beans (Phaseolus vulgaris) and maize; II. Comparison of relay intercropping and simultaneous planting, Field Crops Res. 16, 117-128.

[53] Mucheru-Muna, M., Pypers, P., Mugendi, D., Kung'u, J., Mugwe, J., Merckx, R., Vanlauwe, B. (2010) A staggered maize-legume intercrop arrangement robustly increases crop yields and economic returns in the highlands of Central Kenya, Field Crops Res. 115, 132-139.

[54] Fininsa, C. (1996) Effect of intercropping bean with maize on bean common bacterial blight and rust diseases, Int J Pest Manage 42, 51-54.

[55] Song, Y.N., Marschner, P., Li, L., Bao, X.G., Sun, J.H., Zhang, F.S. (2007) Community composition of ammonia-oxidizing bacteria in the rhizosphere of intercropped wheat (Triticum aestivum L.), maize (Zea mays L.), and faba bean (Vicia faba L.), Biol Fertil Soils 44, 307-314.

[56] Sun, Y.M., Zhang, N.N., Wang, E.T., Yuan, H.L., Yang, J.S., Chen, W.X. (2009) Influence of intercropping and intercropping plus rhizobial inoculation on microbial activity and community composition in rhizosphere of alfalfa (Medicago sativa L.) and Siberian wild rye (Elymus sibiricus L.), FEMS Microbio Ecol 70, 218-226. 


\section{STUDENT AUTHOR}

Heather Miller, a non-traditional student who obtained her bachelor degree in business over 15 years ago, recently returned to school to pursue a new career path in the biological sciences. As an undergraduate at Elmhurst College, she realized a passion for microbial ecology, which was elevated while conducting this research. As such, she applied and has recently been admitted to the graduate college at Michigan State University, where she plans to complete her Ph.D. in microbiology/molecular genetics and environmental toxicology. Her attention will be focused on the microbial ecology of aquatic environments and the effects of eutrophication (e.g. urban and agricultural run-off) and natural resource extraction activities (e.g. high-volume hydraulic fracturing, oil and gas drilling, and mining) on important groups of bacteria capable of bioremediating these events. With this education, she plans to continue research either in an academic, governmental, or private industry setting, helping to identify environmentally sustainable energy extraction processes that consider the vital role of bacteria in maintaining healthy aquatic ecosystems.

\section{PRESS SUMMARY}

Many studies have sought to unravel the mechanisms by which the practice of intercropping corn and bean have benefited both plants, in turn providing explanation of its success and bountiful harvest for thousands of years. Although it is widely accepted that Rhizobium spp., the symbiotic nitrogen-fixing bacteria colonizing legume root systems, facilitates many of these benefits, it was unclear whether other bacteria also played a role. It was hypothesized that when bean and corn were intercropped, the abundance of free-living nitrogen-fixing bacteria would decrease while the abundance of general heterotrophic bacteria remained the same indicating that the planting practice itself triggered a plant-beneficial microbial composition response. Therefore, we prepared and maintained two planting conditions, with and without rhizobium bacteria inoculation, and evaluated the results. 


\title{
Point-Spread Function (PSF) Photometric Analysis of Open Clusters: Melotte 72 \& NGC 2158
}

\author{
Muhammad Awais Mirza \& Zain Rahim ${ }^{2}$ \\ ${ }^{1}$ Institute of Space Technology, Islamabad, Pakistan \\ ${ }^{2}$ Pakistan Space and Upper Atmosphere Research Commission (SUPARCO) Karachi, Pakistan \\ Student:awaismirza786@yahoo.com \\ Mentor: zain_71966@yahoo.com
}

\begin{abstract}
Point Spread Function (PSF) photometry of open star clusters Melotte 72 and NGC 2158 was performed using $g$ and $r$ band data from the Sloan digital Sky Survey (SDSS) Data Release 7. Instrumental magnitudes of stars in both bands were transformed into calibrated magnitudes using standard equation. Color-Magnitude (CM) diagrams were produced and compared with the SDSS isochrones for AB stellar system for different ages and/or metallicities. The objective of this study was to determine the physical parameters (age, distance, metallicity, reddening) of open clusters from CM diagrams. The best fit isochrones were used to estimate cluster parameters. The observed parameters were then compared with the WEBDA data base.
\end{abstract}

\section{KEYWORDS}

PSF Photometry, Melotte 72, SDSS, NGC 2158, Isochrone Fitting, IRAF

\section{INTRODUCTION}

A stellar cluster is a collection of gravitationally bound stars having similar chemical composition, age and are at the same distance from the sun. Born at the same time and from the same molecular cloud, the members of star clusters travel together through space. They are classified as open (galactic) clusters and globular clusters. Open clusters are relatively smaller in size with hundreds to a few thousand stars contained within a diameter of about 30,000 light years across. Stars in open clusters are young (bluish) mostly presented in irregular galaxies or in the galactic plane of spiral ones. Using the techniques of photometry, the important parameters of open clusters can be determined from the light received on earth.

Open clusters are important to study as they help to understand the processes involved in stellar evolution by acting as stellar laboratories ${ }^{1}$ and the assembly and evolution of the Milky Way thin disc. $^{2}$ Two of major variables of stellar evolution, composition and age are identical among all the stars of open clusters - thus open cluster represents a snapshot of hundreds of stars having identical ages and compositions but different masses. In short, an understanding of a star's "life story" can be patched together without having to follow a few individuals from birth to death.

The most basic tool for studying stellar clusters is the Hertzsprung-Russell diagram (HR diagram). It relates the absolute magnitude (luminosity) and temperature (spectral type) of each star in a cluster which then provides a way of looking into the evolution of clusters. Instead of temperature, when color is plotted against absolute magnitude, a Color Magnitude Diagram (CMD) is obtained. CMDs are of great importance to the theories of stellar evolution.

Isochrones are the curves showing same age stars on the HR diagram; as the stars of an open cluster are of same age, these curves can be used to estimate their age. In this research, color magnitude diagrams, obtained using Point Spread Function (PSF) photometry, have been assessed against various isochrones. The best fit isochrone helped estimate the parameters of selected clusters. 
PSF photometry is a more powerful technique than traditional Aperture photometry. PSF photometry is based on the fact that there is same basic profile shape of every star (unlike galaxies or nebulae), i.e., only brightness (amplitude) is different for different stars. Stars are convolved with the telescope optics and (for ground-based telescopes) blurring by the Earth's atmosphere but they all are just images of point sources of light. Hence, a standard profile for all the stars in an image can be created which can be used to find each star's brightness by comparing the standard profile with the profile of that star. ${ }^{3}$

\section{DATA USED}

Physical parameters were estimated for two open clusters: Melotte 72 (Figure 1) and NGC 2158 (Figure 2). Melotte 72 is located at RA $07 \mathrm{~h} 38 \mathrm{~m} 24 \mathrm{~s}$ and DEC $-10^{\circ} 41^{\prime} 00^{\prime \prime}$ (J2000) in the Monoceros constellation with an angular size of 5'. NGC 2158 is in the Gemini constellation at RA 06h 07m 25s and DEC $+24^{\circ} 20^{\prime} 28^{\prime \prime}$ (J2000) having angular size of 4'.

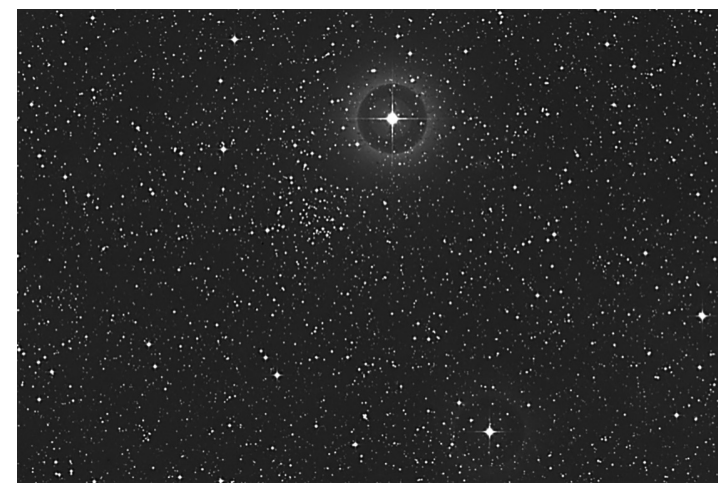

Figure 1. Melotte 72

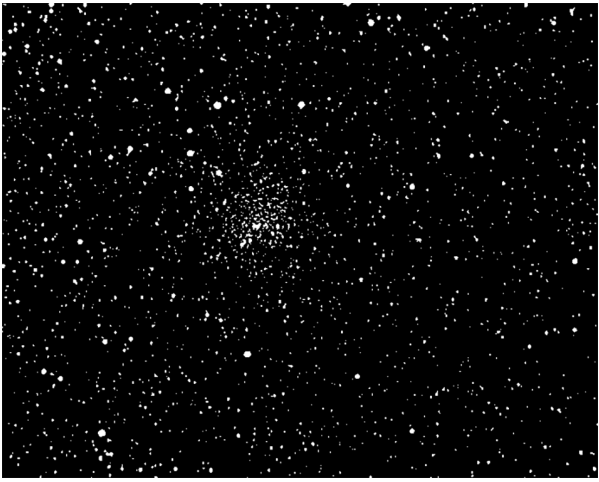

Figure 2. NGC 2158

FITS (Flexible Image Transport System) files of both clusters in $g$ and $r$ filters were downloaded from Sloan digital Sky Survey (SDSS) Data Release 7 FITS. SDSS used 120 megapixel Charge Coupled Device (CCD) with a 2.5-meter telescope at Apache Point in New Mexico, USA. ${ }^{4}$ The images were obtained in $g$ (characteristic wavelength $=468.6 \mathrm{~nm}$ ) and $r$ (characteristic wavelength $=616.5 \mathrm{~nm}$ ) band of the ugriz photometric system. Isochrones concerning to SDSS data were obtained from CMD 2.5 input form at http://stev.oapd.inaf.it/cgi-bin/cmd_2.5 for different ages and metallicities.

\section{METHODOLOGY}

IRAF (Image Reduction and Analysis Facility) was used to process the images of both clusters using PSF photometry. This provided the $g$ and $r$ band instrumental magnitudes of individual stars of each cluster. Ds9 was interfaced with IRAF to visualize images while performing photometry tasks. Stars in both clusters were then cross matched using Tool for Operations on Catalogues and Tables (TOPCAT). Finally, a single file containing the instrumental magnitudes of stars in a cluster was obtained. The instrumental magnitudes were calibrated and a CMD plot was plotted between $g$ and $g-r$ which was then fitted with appropriate isochrones. 


\section{i. Determination of Instrumental Magnitudes using PSF}

Using the imexamine task in IRAF, the researchers determined the Full Width Half Maximum (FWHM) of some of stars and the standard deviation of background sky in both images and calculated their average values. These values were used to set centerpars, daopars, datapars, findpars, fitsktpars, photpars in IRAF. The procedure for generating PSF is as follows:

- 25 to 50 candidate stars were selected using 'pstselect' task

- Using 'psf' task, among the pst starlist only those were selected having similar surface plots

- In the next step the PSF using 'nstar' and 'substar' tasks was refined

- 'psf' was run again which produced final PSF

- Using 'allstar' task, the final built psf was fitted to all the stars in the image

Figures $\mathbf{3}$ and $\mathbf{4}$ present the radial profile density and surface plot of the PSF model.

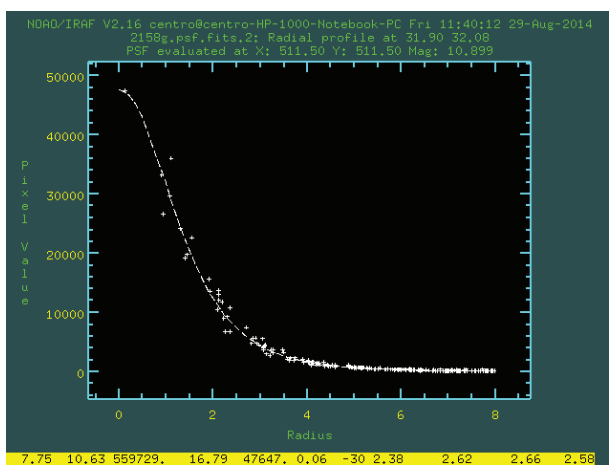

Figure 3. Radial Profile Density of PSF Model

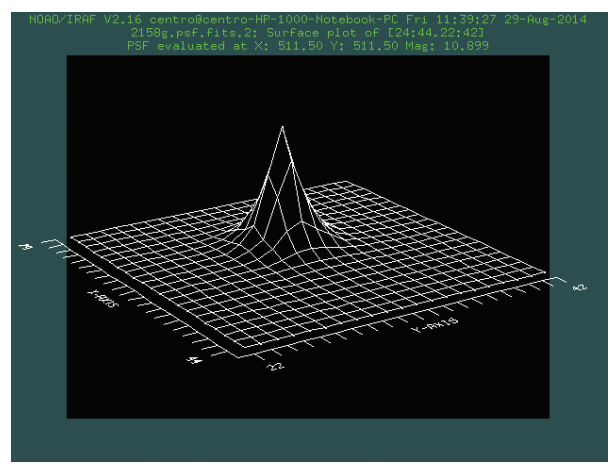

Figure 4. Surface Plot of PSF Model

Using pdump task, a text file containing coordinates of each star and its corresponding instrumental magnitude was obtained.

\section{ii. Calibration}

The procedure defined in the previous section was repeated separately for $g$ and $r$ filter FITS images of both clusters so there were two files containing the data of stars for each respective filter. As there was a difference between numbers of stars detected by both filters, cross-matching of individual stars in each band was performed to select only those stars which were detected in both bands. For this purpose TOPCAT was used which produced a single data file for each cluster.

Instrumental magnitudes of common stars in both filters of each cluster were calibrated into corresponding calibrated magnitudes using

where

$$
m=m_{0}+\left(m_{\text {ins }}-25\right)-k x
$$

$m_{0}=$ constant depending on the considered filter

$m_{\text {ins }}=$ instrumental magnitude

$k=$ atmospheric extinction coefficient

$x=$ air mass

and 25 is a constant applied to the instrumental magnitude values to make them positive.

The values of the aforementioned parameters for both clusters have been taken from the photometry data file from SDSS and presented in Table 1. 


\begin{tabular}{|c|c|c|c|c|c|c|}
\hline & \multicolumn{3}{|c|}{ Melotte 72 } & \multicolumn{3}{c|}{ NGC 2158 } \\
\cline { 2 - 7 } & $m_{0}$ & $k$ & $x$ & $m_{0}$ & $k$ & $x$ \\
\hline$G$ & 24.3748 & 0.17412 & 1.3907 & 24.5667 & 0.1506 & 1.0235 \\
\hline$R$ & 24.0401 & 1.06033 & 0.1068 & 24.5667 & 0.0916 & 1.02353 \\
\hline
\end{tabular}

Table 1. Photometric parameters for both open clusters

\section{iii. CMD Plotting and Isochrones Fitting}

MATLAB was used to plot Color-magnitude diagrams of both open clusters which were then fitted with appropriate isochrones. Isochrone fitting was started using an educated guess based on age and metallicity ranges of open clusters. Several same age isochrones with different metallicities and vice versa were tested. Best fitted isochrones were then used to estimate the parameters of the star clusters. Figure 5 shows the isochrones fitted color-magnitude diagram of Melotte 72 and NGC 2158.
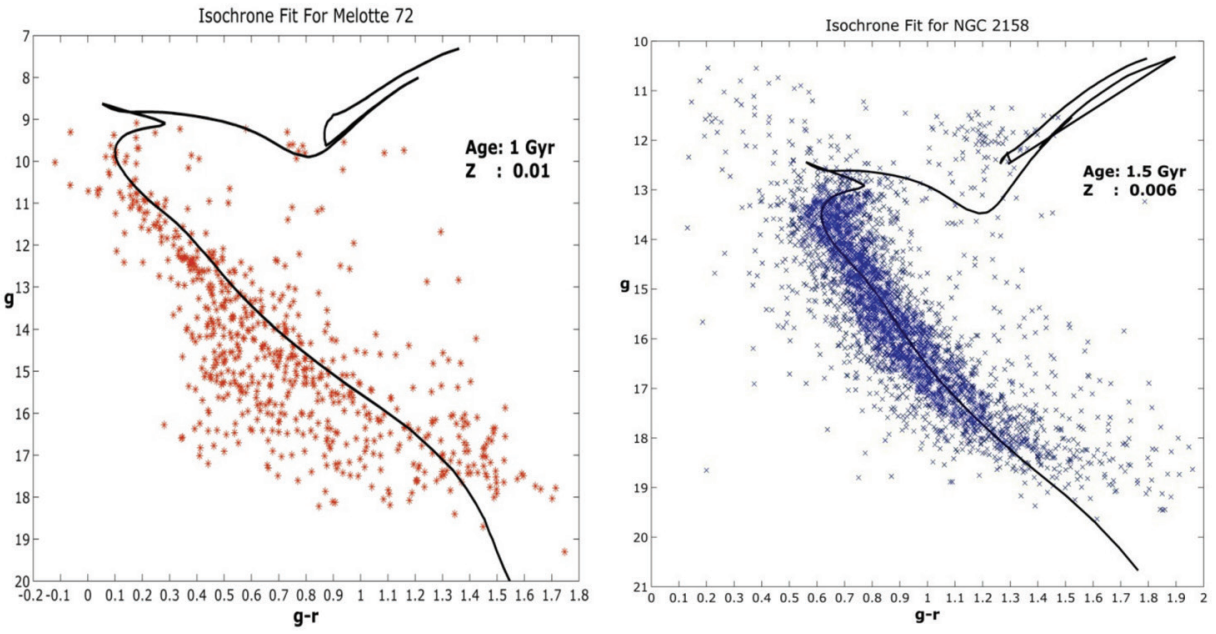

Figure 5. Isochrone fitted color magnitude diagrams of Melotte 72 and NGC 2158

\section{iv. Calculations}

Distances of both open clusters were calculated using the following equation:

$$
(m-M)=5 \log d-5
$$

Where $(m-M)$ is distance modulus and $d$ is distance in parsecs. In this case, distance modulus was the difference between $g$ coordinates of turn-off values of cluster data and the best fit isochrones. Similarly, reddening was obtained from the difference in $g-r$ coordinate of two turn-off values. Table 2 shows the values of distance modulus, reddening, metallicity and the distance determined for both open clusters. 


\begin{tabular}{|c|c|c|c|c|}
\hline & \multicolumn{2}{|c|}{ Melotte 72 } & \multicolumn{2}{c|}{ NGC 2158 } \\
\cline { 2 - 5 } & Our Findings & At WEBDA & Our Findings & At WEBDA \\
\hline Age $(G y r)$ & $1 \mathrm{Gyr}$ & 0.60 & 1.5 & 1.05 \\
\hline Metallicity $(Z)$ & 0.01 & 0.000 & 0.006 & -0.023 \\
\hline Distance Modulus $\left(V-M_{v}\right)$ & 8.242 & 13.01 & 11.406 & 14.64 \\
\hline Reddening $E(V-B)$ & 0.199 & 0.20 & 0.65 & 0.360 \\
\hline Distance $(p c)$ & 445.04 & 3000 & 1910.73 & 5071 \\
\hline
\end{tabular}

Table 2. Photometric parameters for both open clusters

\section{RESULTS/CONCLUSIONS}

Table 2 suggested a little higher values for the age of both open clusters when compared with those at WEBDA. It was $0.40 \mathrm{Gyr}$ higher for Melotte 74 and $0.45 \mathrm{Gyr}$ for NGC 2158. Similarly, reddening values were found acceptable for Melotte 72 while a bigger difference was seen for NGC 2158. It was noticed that we underestimated the distance of both open clusters with a larger difference. 


\section{ACKNOWLEDGEMENTS}

The authors are thankful to Mr. Ghulam Mustafa, Mr. Abdul Salam and Ms. Ausima Sultan for their discussion and help during the work.

\section{REFERENCES}

[1] Kalirai, J. S. and Richer, H. B. (2010) Star clusters as laboratories for stellar and dynamical evolution, PTRSL 368, 755-782. doi:10.1098/rsta.2009.0257.

[2] Bland-Hawthorn, J., Krumholz, M. R. and Freeman, K. (2010) The Long-Term Evolution of the Galactic Disk Traced by Dissolving Star Clusters, ApJ 713, 166-179. doi:10.1088/0004-637X/713/1/166.

[3] Harris, W. E. Stellar Photometry Using IRAF, McMaster University Department of Physics \& Astronomy, http://www.physics.mcmaster.ca/ harris/daophot_irafmanual.txt (accessed Sep 09, 2014)

[4] Gunn, J. E., Siegmund, W. A., Mannery, E. J., Owen, R. E., Hull, C. L., Leger, R. F., Carey, L. N., Knapp, G. R., York, D. G., Boroski, W. N., Kent, S. M., Lupton, R. H., Rockosi, C. M., Evans, M. L., Waddell, P., Anderson, J. E., Annis, J., Barentine, J. C., Bartoszek, L. M., Bastian, S., Bracker, S. B., Brewington, H. J., Briegel, C. I., Brinkmann, J., Brown, Y. J., Carr, M. A., Czarapata, P. C., Drennan, C. C., Dombeck, T., Federwitz, G. R., Gillespie, B. A., Gonzales, C., Hansen, S. U., Harvanek, M., Hayes, J., Jordan, W., Kinney, E., Klaene, M., Kleinman, S. J., Kron, R. G., Kresinski, J., Lee, G., Limmongkol, S., Lindenmeyer, C. W., Long, D. C., Loomis, C. L., McGehee, P. M., Mantsch, P. M., Neilsen, E. H., Neswold, R. M., Newman, P. R., Nitta, A., Peoples, J.,Pier, J. R., Prieto P. S., Prosapio, A., Rivetta, C.,Donald, Schneider, P., Snedden, S., and Wang, S. (2006) The 2.5 m Telescope of the Sloan Digital Sky Survey, ApJ 131, 2332-2359.

\section{ABOUT THE STUDENT AUTHOR}

Muhammad Awais Mirza is a junior undergrad student at Department of Space Science, Institute of Space Technology (IST), Islamabad. He undertook this research during summer internship at SUPARCO - the national space agency of Pakistan. A space nut and an excellent team player, Awais is working as the head of Astronomy and Astrophysics wing of IST Space Society. His great passion inspires him to further engage with the topic in the framework of a professional career.

\section{PRESS SUMMARY}

Star Clusters are the collections of stars, born at same time, traveling together through space. After processing images (from a 2.5 meter telescope) of two star clusters, we obtained their color (temperature) vs. luminosity graphs. Using these graphs, we estimated the parameters like distance and age of these clusters. Finally, the estimated parameters were compared with the literature. 


\title{
Synthesis of a MUC1 Mucin Cyclic Dimer Peptide and Its Antibody Binding Properties as Revealed by STD-NMR
}

\author{
Cheng Her \& Thao Yang* \\ Department of Chemistry, University of Wisconsin - Eau Claire, Eau Claire, WI \\ Student: herxx373@umn.edu \\ Mentor: yangt@uwec.edu
}

\begin{abstract}
In a previous study we showed that the shortened MUC1 mucin peptide GVTSAPD could bind monoclonal antibody (mAb). We proceeded on to make a cyclic peptide of the same sequence to see if it would be more effective in binding antibody. We were able to synthesize and isolate two different cyclic mucin peptides: 1) a monomer cyclic peptide with sequence GVTSAPD which we did not study due to difficulties in achieving homogeneity, and 2) a dimer cyclic peptide with sequence GVTSAPDGVTSAPD that was successfully isolated and studied. We describe here the results of the dimer cyclic peptide-antibody interactions obtained by Saturation Transfer Difference NMR (STDNMR). The results indicated that the protons of all residues experienced STD effects, notably being more pronounced at Pro, Val, Ala and Asp compared to the linear peptide GVTSAPD. The Pro residue exhibited STD peaks for all its side chain protons with stronger intensity at $\mathrm{ProH} \gamma$ while Ala, Val and Thr are localized to methyl groups.
\end{abstract}

\section{KEYWORDS}

Mucl Antibody Recognition Epitope, STD NMR, Mucin Peptide-antibody Interactions, Cyclodimer Peptide.

\section{INTRODUCTION}

The mucin protein, encoded by the MUC1 gene, is an epithelial transmembrane glycoprotein of which the extracellular domain exhibits a repeating 20 amino acid sequence (GVTSAPDTRPAPGSTAPPAH) known as the Tandem Repeat Domain (TRD)..$^{1-3}$ The TRD contains five O-linked glycosylation sites (underlined) to which the attached carbohydrates aid in carrying out the normal cellular functions of MUC1 mucin, which includes binding of pathogens as a protective system, cell signaling, and lubrication/hydration of the epithelial surfaces. ${ }^{1,2}$ Studies have shown that the MUC1 mucin amino acid sequence of cancer and normal cells of the epithelia are identical. ${ }^{2}$ Human adenocarcinomas exhibit overexpression of MUC1 mucin of truncated oligosaccharide structures at the TRD region which makes it distinguishable from normal epithelial cells. ${ }^{1-5}$ These discoveries are significant to the extent that reduced glycosylation of MUC1 mucin allows access of the TRD to the immune system producing low level immunity. ${ }^{6,7}$ Expression of MUC1 mucin and its glycosylation content of the TRD have been studied extensively and suggested to hold high therapeutic potentials against cancers. ${ }^{8,9}$

The structural differences between the two forms of MUC1 mucins have generated interest and led effort in utilizing MUC1 mucin in immunotherapy of cancer. ${ }^{10,11}$ It has been reported that antibody responses specific for MUC1 mucin have been detected in patients of breast, pancreatic and ovarian cancers, and other studies have discovered a binding site of these antibodies to the MUC1 mucin to be at the sequence PDTRP at the TRD. ${ }^{2-4}$ In a previous study we have determined that the linear sequence GVTSAPD is also capable of binding to mAb (6A4) from mouse with significant interactions at the proline residue. ${ }^{12,13}$ This demonstrates that the $\mathrm{mAb}$ raised against specific antigen epitope can interact with peptides with similar structure to its binding interface, and that 
absolute specificity is not a requirement, which may offer, as a general strategy, more room for improvement in the design of variety of antigenic agents to induce the same kind but more effective immunity in the area of MUC1 mucin based cancer immunotherapy. In an attempt to develop a mucin peptide epitope that can interact effectively with MUC1 mAb that may potentially be capable of inducing sufficient immunity without being compromised by cellular enzymatic reactions, we proposed to design cyclic peptides and other non-natural amino acid peptide derivatives based on the known antigenic sequence of the MUC1 mucin. Cyclic peptides based on known epitope sequence may give increased peptide-antibody affinity, which may afford them as additional antigenic agents. Herein we report the synthesis and preliminary results of the antibody binding properties of a cyclic dimer peptide, which has its sequence repeated twice based on the sequence GVTSAPD.

\section{ABBREVIATIONS}

DCM, dichloromethane; DIPEA, diisopropylethylamine; DMF, dimethylformamide; Fmoc, $\mathrm{N}$ - $\alpha$-9-fluorenylmethoxycarbonyl; HBTU, O-(Benzotriazol-1-yl)-N,N,N',N'-tetramethyluronium hexafluorophosphate; HOBt, N-hydroxybenzotriazole; ODmab, 4\{N-[1-(4,4-Dimethyl-2,6dioxo-cyclohexylidene)-3-methylbutyl]-amino\}benzyloxy; PyBOP, benzotriazole-1-yl-oxy-trispyrrolidino-phosphonium hexafluorophosphate; TIC, total ion current; Wang resin, $p$-alkoxybenzyl alcohol-linked Wang resin; $\mathrm{u}, \mathrm{m} / \mathrm{z}$ unit.

\section{EXPERIMENTAL PROCEDURES}

SPPS of Cyclic Mucin Peptide.

The peptide was synthesized via standard Fmoc-chemistry by Solid Phase Peptide Synthesis (SPPS). ${ }^{14}$ For specific details on building the peptide chain and cleavage, see references 12 and 13. ${ }^{12,13}$ The pre-loaded resin used was Fmoc-Asp (Wang resin LL)-ODmab (Novabiochem). After the entire sequence has been synthesized, the ODmab group protecting the $\alpha$-carboxyl group at the C-terminus of the peptide was removed with $2 \%$ hydrazine $(\mathrm{v} / \mathrm{v})$ in DMF and then vacuum dried for cyclization. The cyclization reagents consisted of a mixture of $4 \mathrm{x}$ excess of resin of PyBOP, $\mathrm{HOBt}$ and 2x excess of DIPEA. The head-to-tail cyclization strategy was carried out for $24 \mathrm{hrs}$. Scheme 1 summarizes the general strategy for synthesis of the cyclic mucin peptide. ${ }^{15,16}$

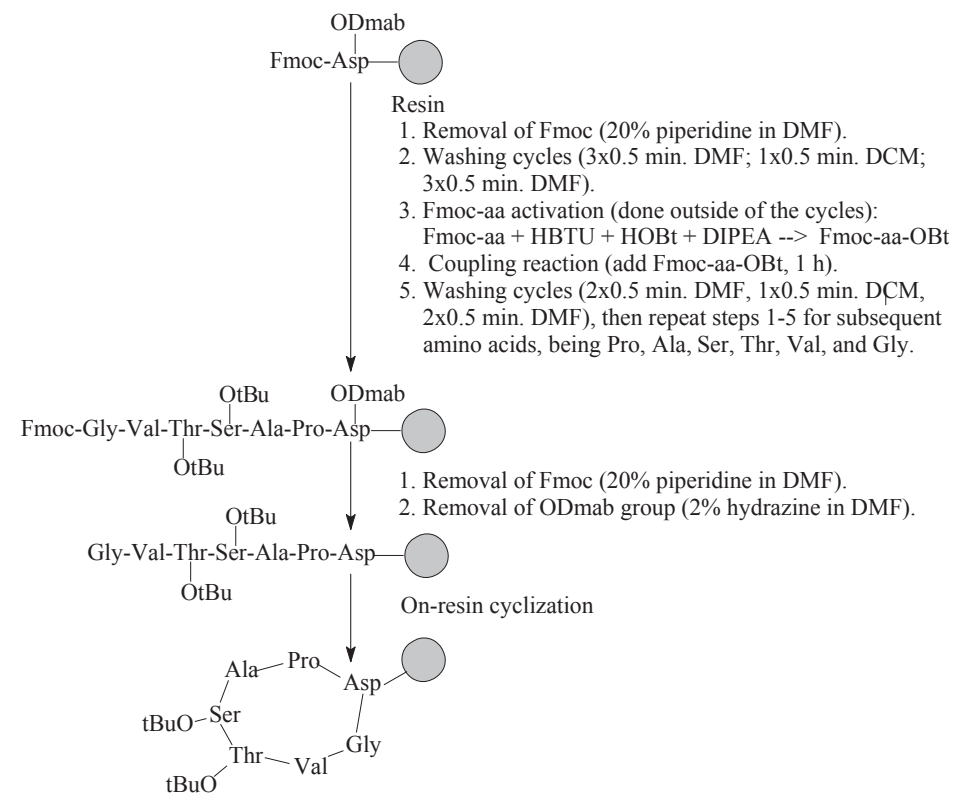

Scheme 1. General scheme for the strategy of cyclic peptide synthesis. The Asp side chain is linked to the resin; the ODmab group is on the a-CO2-group of Fmoc-Asp-resin 


\section{HPLC Purification of Peptides.}

Peptide was purified using a Varian Prostar HPLC. ${ }^{12,13}$ The method used a reverse phase preparative $\mathrm{C}_{18}$ column (10x250 mm, $10 \mu \mathrm{m}$, Grace Vydac) with acetonitrile (ACN) as the organic mobile phase and monitored at $220 \mathrm{~nm}$. The flow rate was set at $2 \mathrm{~mL} / \mathrm{min}$ with a gradual increase in ACN from $0-35 \%$ at the $30 \mathrm{~min}$. mark. Fractions were collected manually and freeze-dried for storage and subsequent NMR experiments. The buffers used in the HPLC were $1 \%$ ACN, $0.1 \%$ HOAc (acetic acid), $98.9 \%$ water (buffer A), and $1 \%$ water, $0.1 \%$ HOAc, $98.9 \%$ ACN (buffer B).

\section{LC-MS Analysis.}

The freeze-dried solid peptide sample was analyzed for presence of the desire product with an Agilent Mass Spectrometer (Agilent Technologies Time-of-Flight LC/MS 6210). The buffers used in the LC-MS analysis were the same as in HPLC. The conditions used in the LC-MS were: $0.5 \mathrm{~mL} /$ min flow rate, $5-100 \% \mathrm{~B}$ in 20 minute buffer gradient, $20 \mathrm{~min}$. acquisition time followed by a $4 \mathrm{~min}$. post run, $\mathrm{C}_{18}$ column $\left(4.6 \mathrm{x} 150 \mathrm{~mm}, 3.5 \mu \mathrm{m}, \mathrm{ZORBAX}\right.$ Extend- $\left.\mathrm{C}_{18}\right)$ and ESI positive mode. ${ }^{12,13}$

\section{NMR Experiments.}

All NMR experiments were performed using a $400 \mathrm{MHz}$ Bruker Avance Spectrometer. Generally, peptide samples were at 3-5 mM, in $20 \mathrm{mM}$ phosphate buffer, $5 \mathrm{mM} \mathrm{NaCl}$, pH 5, 90\% $\mathrm{H}_{2} \mathrm{O}$, $10 \% \mathrm{D}_{2} \mathrm{O}$, at $7^{\circ} \mathrm{C}$. The $2 \mathrm{D}$ NMR (TOCSY and ROESY, Bruker TopSpin software v. 2.0.5) data were collected for proton assignments. The TOCSY data give the spin system of each amino acid residue; thus, ${ }^{1} \mathrm{H}-{ }^{1} \mathrm{H}$ connectivity through bond within each amino acid residue. The ROESY data allow for ${ }^{1} \mathrm{H}-{ }^{1} \mathrm{H}$ connectivity between the sequential amino acids as well as through space long range interactions between two ${ }^{1} \mathrm{H}^{\prime}$ s that may be close spatially within $5 \AA{ }^{17,18}$ Suppression of $\mathrm{H}_{2} \mathrm{O}$ signal in the 2D NMR spectra was accomplished by the 3-9-19 WATERGATE pulse sequence with gradients applied at each cycle of the 2D TOCSY or ROESY pulse sequence. ${ }^{19}$

The STD NMR technique was used to study the binding interactions of peptide ligand with antibody. ${ }^{13,20,21}$ Mixture samples of $0.01 \mathrm{mM}$ of mouse Mucl specific monoclonal antibody IgG1 (6A4 clone, $\mathrm{Mr}_{\mathrm{r}} 122 \mathrm{kDa}$, Genway Biotech) with $1 \mathrm{mM}$ of peptide ligand were used (peptide:antibody ratio $=100: 1$ ). The STD NMR data were collected with peptide-antibody mixtures made up in $20 \mathrm{mM}$ phosphate buffer, $5 \mathrm{mM} \mathrm{NaCl}, \mathrm{pH} 7,100 \% \mathrm{D}_{2} \mathrm{O}$, at $7^{\circ} \mathrm{C}$ with presence of considerable $\mathrm{HDO}$ signal. At $\mathrm{pH} 7$, the aliphatic ${ }^{1} \mathrm{H}$ NMR resonances do not deviate unambiguously from those at $\mathrm{pH} 5$. In the STD NMR technique, two NMR spectra were collected; one with the on-resonance frequency set at $-2 \mathrm{ppm}$ where there are no peptide ${ }^{1} \mathrm{H}$ signals but antibody ${ }^{1} \mathrm{H}$ signals are present, and the other with the off-resonance frequency set at $+40 \mathrm{ppm}$ where there are no ${ }^{1} \mathrm{H}$ signals from either ligand or antibody. The saturation time was $2 \mathrm{~s}$. The on-resonance spectrum intensity (ISAT) is subtracted from the off-resonance spectrum intensity $\left(\mathrm{I}_{0}\right)$ to obtain the STD NMR spectrum (ISTD $=\mathrm{I}_{0}-\mathrm{I}_{\mathrm{SAT}}$ ); the amplification factor (ASTD) is calculated by the equation: ASTD $=\left(I_{S T D} / \mathrm{I}_{0}\right) \mathrm{x}[\mathrm{L}] \mathrm{t} /[\mathrm{P}]$, where $[\mathrm{L}] \mathrm{t}$ is the total peptide concentration and $[\mathrm{P}]$ is the antibody concentration. If there is any proton on the ligand that directly binds the antibody, it will show a positive STD signal; the proton closer to the antibody will receive more saturation transfer effect and its ISTD peak intensity will be higher. ${ }^{21}$ If the peptide does not bind the antibody (e.g., no ${ }^{1} \mathrm{H}$ 's on the peptide experienced any saturation transfer effect), the resultant subtracted spectrum (STD spectrum) will have no signals (flat line). ${ }^{16,17}$ The STD NMR spectrum was used to identify which proton on which amino acid residue on the peptide has direct binding at the antibody binding interface. 


\section{RESULTS}

Synthesis.

Synthesis of the cyclic mucin peptide produced two major products of interest. The proposed reactions that led to the two peptides are illustrated on Scheme 2, where an intrachain head-to-tail reaction led to the monomer cyclic peptide (mass $627.31 \mathrm{Da}$ ), and an interchain head-to-tail reaction led to the dimer cyclic peptide (mass $1254.60 \mathrm{Da}$ ).

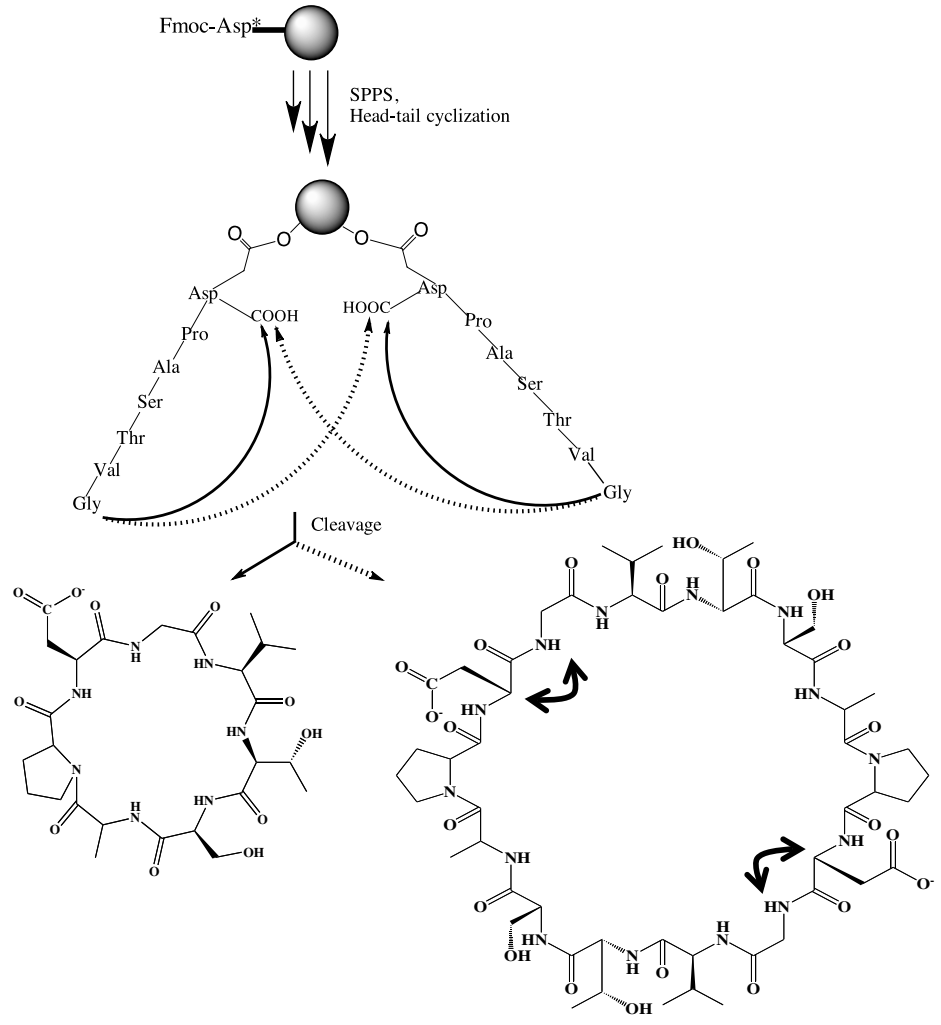

Scheme 2. Peptide cyclization mechanisms resulting in two different cyclic peptides: a cyclomonomer peptide via intrachain head-to-tail reaction (left, solid pathway, mass $627.31 \mathrm{Da}$ ) and a cyclodimer peptide via interchain head-to-tail reaction (right, dashed pathway, mass $1254.60 \mathrm{Da}$ ). “* ” signifies ODmab group on the $\alpha$-CO2-group of Fmoc-Asp-resin; curved double headed arrows indicate NOE's that would be observed on the cyclodimer. 


\section{HPLC Analysis.}

HPLC purification following the synthesis of the mucin cyclic peptide produced three separate peaks at approximate retention times of 19.7, 23.6 and 34.0 min. Figure 1 shows the HPLC chromatogram of the crude peptide sample in which the cyclic monomer peptide and cyclic dimer peptide were isolated and purified. Subsequent LC-MS analysis of the collected pools of peaks a, b and c from HPLC (Figure 1, peaks a, $\mathbf{b}$, and $\mathbf{c}$ ) showed that these three peaks corresponded respectively to the cyclic monomer peptide of mass $628.31 \mathrm{Da}$ (peak a), the cyclic dimer peptide of mass $1255.60 \mathrm{Da}$ (peak b) and an elution peak (peak c) that contained no products of interest. There was no significant elution before the $20 \mathrm{~min}$. mark as the amount of ACN being pumped through was relatively low. Most of the contaminants and unwanted side products eluted after the cyclic dimer peak at 24 min. when the amount of organic solvent being pumped through was over $30 \%$. The peaks of interests (retention times 19.7 and $23.6 \mathrm{~min}$.) were collected, freeze-dried and re-purified by HPLC a second time and their chromatograms showed a single peak with the expected retention times (data not shown) before they were used for later experiments.

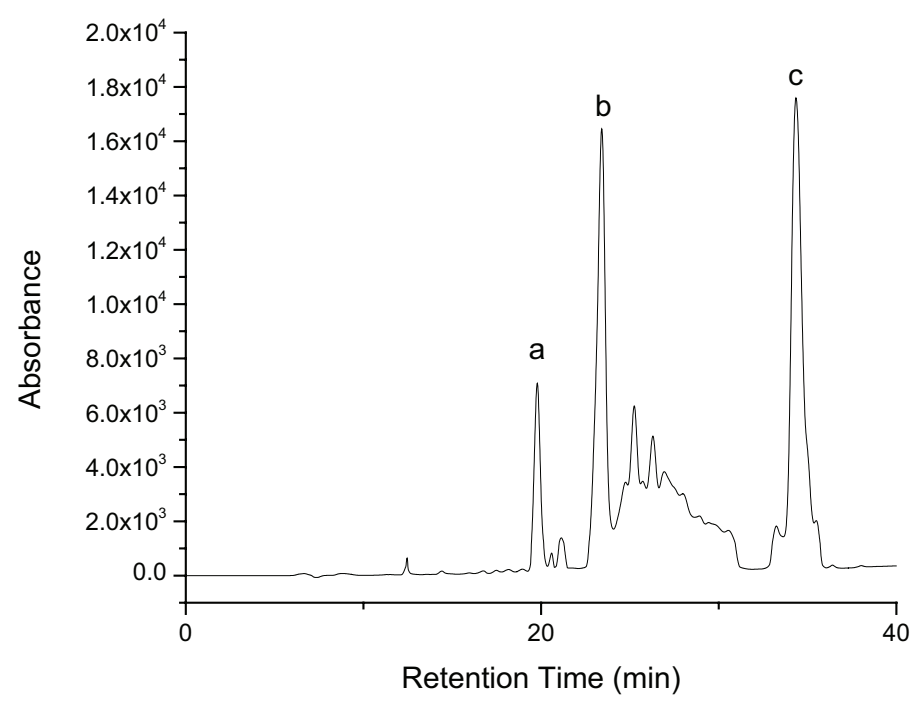

Figure 1. HPLC purification of crude peptide sample. The HPLC chromatogram was obtained from a $500 \mu \mathrm{L}$ concentrated sample injection. Acquisition time was $40 \mathrm{~min}$. with a $5 \mathrm{~min}$. post equilibrium/hold and monitored with UV light. Chromatogram was extracted from wavelength of $220 \mathrm{~nm}$; (a) peak corresponding to the cyclic peptide of interest with mass of $[\mathrm{M}+\mathrm{H}]^{+}=628.31 \mathrm{Da}$, (b) peak corresponding to the dimer cyclic peptide of interest with mass of $\left[\mathrm{M}_{\text {dimer }}+\right.$ $\mathrm{H}]^{+}=1255.60 \mathrm{Da}$, and (c) impurities found in the crude peptide sample that did not contain any products of interest. 


\section{LC-MS Analysis.}

LC-MS analysis of the crude peptide sample following synthesis showed the presence of two dominant masses in the finished product mixture. Figure $\mathbf{2}$ shows the chromatogram of the LC-MS analysis of the crude peptide (Figure 2B) and a purified dimer peptide (Figure 2A).

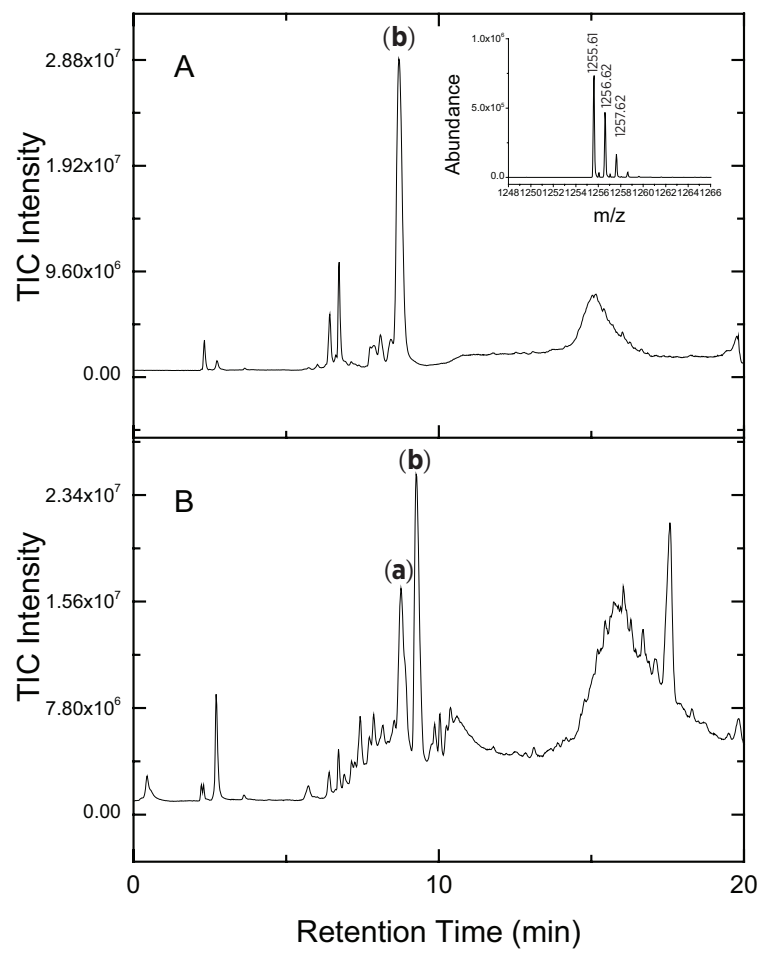

Figure 2. LC-MS chromatograms of purified (A) and crude (B) peptide samples following completion of peptide synthesis and extraction. Peaks of interest were (a) product of mass $[\mathrm{M}+\mathrm{H}]^{+}=628.31 \mathrm{Da}$ and $(\mathbf{b})$ product of mass $[\mathrm{Mdimer}+$ $\mathrm{H}]^{+}=1255.60 \mathrm{Da}$; inset on Figure 2A shows the isotopic m/z value of this singly charged ion at $1255.61 \mathrm{u}, 1256.62 \mathrm{u}$ and $1257.62 \mathrm{u}$.

Two distinct ions of $\mathrm{m} / \mathrm{z}$ values of $628.31 \mathrm{u}$ and $1255.60 \mathrm{u}$ in the TIC eluted at approximately 8.75 min. and 9.25 min., respectively (Figure 2B). These two ions were indicative of a monomer cyclic GVTSAPD $\left(\left[\mathrm{M}+\mathrm{H}^{+}\right]_{\text {calc }}=628.64 \mathrm{u}\right)$ and a dimer cyclic GVTSAPDGVTSAPD $\left(\left[\mathrm{M}_{\text {dimer }}+\mathrm{H}^{+}\right]_{\text {calc }}\right.$ $=1255.29 \mathrm{u}$ ). The other peaks in the TIC did not contain any masses of interest and were accepted as contaminants or side products from the synthesis. The 1255.60 Da peptide collected from HPLC (from Figure 1, peak $\mathbf{b}$ ) was reanalyzed by LC-MS and showed a major peak at the expected retention time of $9.25 \mathrm{~min}$. (Figure 2A). The ion at $1255.60 \mathrm{u}$ could not have been made up of an adduct of two single cyclic monomers, which would also have the same $\mathrm{m} / \mathrm{z}$ value of $1255.60 \mathrm{u}$ $\left(\left[2 \mathrm{M}_{\text {monomer }}+\mathrm{H}^{+}\right]=1255.60 \mathrm{u}\right)$, because we have collected that peak from HPLC and have repurified it by HPLC the second time, and we did not observe an equilibrium of monomer-dimer adduct formation, instead we obtained only a single peak at the expected retention time. This was true in both cases when we used both purified samples and reran the HPLC and LC-MS. If the ion at $1255.60 \mathrm{Da}$ was due to a dimer adduct of two cyclic monomers, we would expect the cyclic monomer peak to be present or increasing in the reruns of HPLC and LC-MS experiments, however these were not the case. It is not entirely unexpected to obtain dimer or oligomers during peptide chain cyclization, such cyclodimerization (or cyclo-oligomerization) had previously been observed. ${ }^{16,22}$ We obtained the cyclic dimer using the particular resin Fmoc-Asp(Wang resin LL)-ODmab (Novabiochem). 
To minimize dimer formation one could select a resin with lower equivalent of resin loading; however, in the present case, it is a blessing that a cyclic dimer peptide was formed and isolated and found to have more interactions with the antibody, which is a goal sought after.

\section{NMR Results.}

The peptide corresponding to a mass of $628.31 \mathrm{Da}$ (Figure 2B, peak a) was not selected for antibody binding experiments or further 2D NMR experiments because after several trials of synthesis and HPLC re-purifications, while it eluted as a single peak, its ${ }^{1} \mathrm{H}$ 1D NMR spectrum always showed a set of more than four methyl groups present (i.e., eight methyl groups). This suggested a mixture of two enanteomeric peptides, most likely, due to epimerization, which could not be separated by the HPLC column used. The 1D NMR spectrum of the peptide corresponding to a mass of $1255.60 \mathrm{Da}$ (Figure 2B, peak b) showed four set of methyl groups as expected for a dimer cyclic peptide in which one half of the molecule is identical to the other half but in reverse order. Thus, only the peptide corresponding to a mass of $1255.60 \mathrm{Da}$ was selected for further experiments (i.e., antibody binding studies and 2D NMR experiments). Protons assignments were achieved by the 2D NMR TOCSY and ROESY experiments. Table 1 shows the ${ }^{1} \mathrm{H}$ chemical shift assignments for the cyclic dimer peptide.

\begin{tabular}{|c|c|c|c|c|}
\hline \multirow{2}{*}{} & Residue & \multicolumn{3}{|c|}{ Chemical Shifts (ppm) } \\
\cline { 2 - 5 } & $\mathrm{NH}$ & $\alpha \mathrm{H}$ & $\beta \mathrm{H}$ & Others \\
\hline $\mathrm{G}$ & 8.339 & $3.880,4.059$ & & $\gamma \mathrm{CH}_{3} 0.922$ \\
\hline $\mathrm{V}$ & 8.149 & 4.225 & 2.107 & $\gamma \mathrm{CH}_{3} 1.205$ \\
\hline $\mathrm{T}$ & 8.612 & 4.385 & 4.217 & \\
\hline $\mathrm{S}$ & 8.415 & 4.481 & 3.836 & \\
\hline $\mathrm{A}$ & 8.528 & 4.606 & 1.363 & \\
\hline $\mathrm{P}$ & & 4.396 & $2.028,2.316$ & $\mathrm{H} \gamma, \gamma^{\prime} 1.951,2.028 ; \mathrm{H} \delta, \delta^{\prime} 3.664,3.821$ \\
\hline $\mathrm{D}$ & 8.278 & 4.529 & $2.695,2.781$ & \\
\hline
\end{tabular}

Table 1. ${ }^{1} \mathrm{H}$ chemical shifts of cyclic dimer peptide obtained from the TOCSY spectrum. Peptide concentration was $5 \mathrm{mM}$ in $20 \mathrm{mM}$ phosphate buffer, $5 \mathrm{mM} \mathrm{NaCl}$, pH 5, 90\% $\mathrm{H}_{2} \mathrm{O}, 10 \% \mathrm{D}_{2} \mathrm{O}$ at $7{ }^{\circ} \mathrm{C}$. Chemical shifts were referenced to the $\mathrm{HDO}$ peak set at $4.970 \mathrm{ppm}$ at $7^{\circ} \mathrm{C}$.

We were able to successfully map out the peptide backbone NOE peaks by the $2 \mathrm{D}{ }^{1} \mathrm{H}$ NMR ROESY data to confirm the correct amino acid sequence. Figure 3 shows the NOE sequential assignments of $\alpha \mathrm{H}_{\mathrm{i}}-\mathrm{NH}_{(\mathrm{i}+1)}$ along the peptide backbone. A unique strong NOE peak bridging the interaction between the GlyNH of chain 1 to the AspHa of chain 2 was observed (Figure 3), attesting the presence of a cyclic peptide. In a linear peptide the NOE 'GlyNH of chain 1 to AspHa of chain 2 ' would not be observed because of the large distance between those two H's and broadening of the NH peak. Additionally, we observed a very weak NOE between the GlyNH and AspNH, further supporting the cyclic structure of the peptide. 


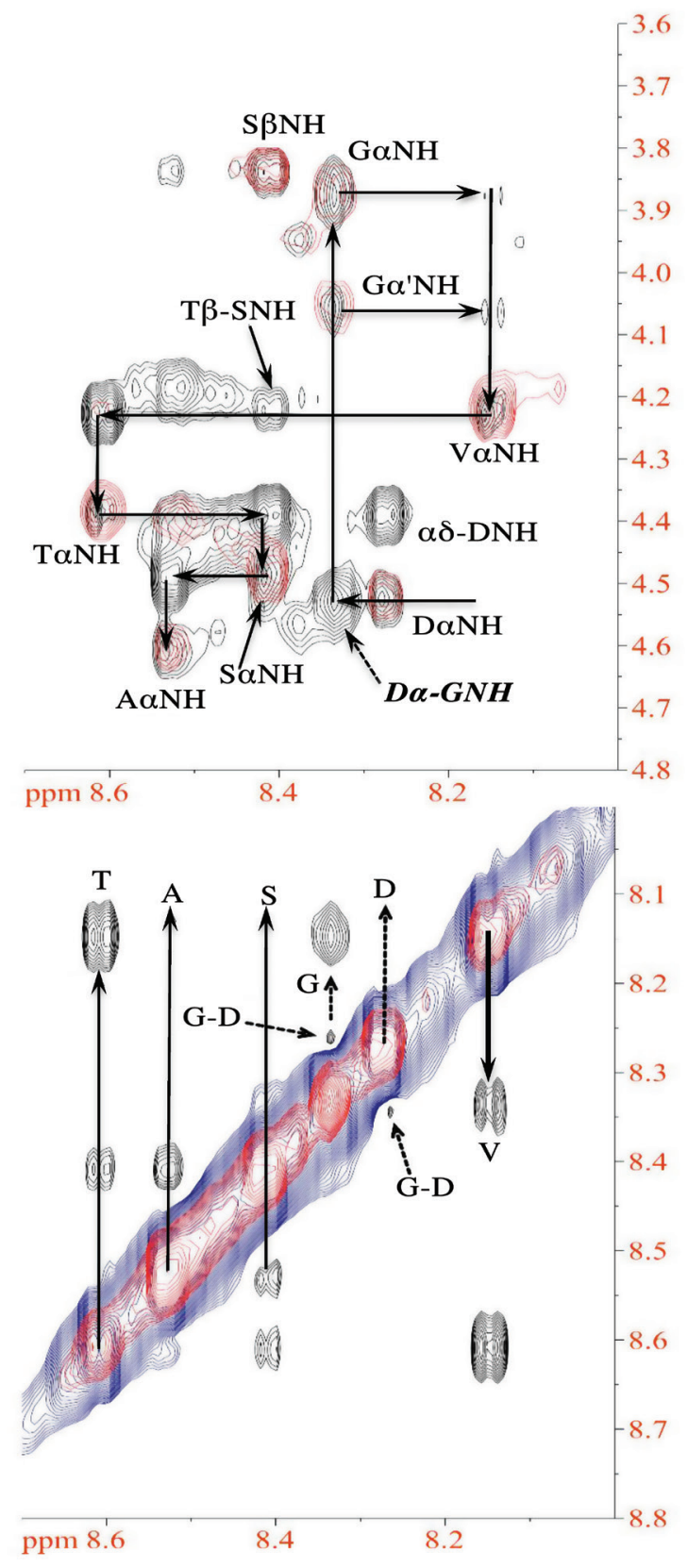

Figure 3. 2D NMR spectra of $\mathrm{CH} \alpha-\mathrm{NH}$ (top) and NH-NH (bottom) regions of cyclic dimer peptide with sequence cyclo-GVTSAPDGVTSAPD. Black color contours represent ROESY data and red color, TOCSY data. Cross-peaks between Ha's and NH's within each residue are indicated; in addition, a unique NOE peak bridging the interaction between adjacent Asp of chain 2 and Gly of chain 1 (AspHa-GlyNH) confirms the cyclic nature of this molecule (bold label). Arrows show the NOE sequential assignment of the peptide backbone starting with Asp and ending at Ala. Non-label peaks that are not in the backbone connectivity arose from NOE's within the same peptide and from spin systems that we believe were from a minor isomeric peptide. 


\section{STD NMR Results.}

Figure 4 shows the ${ }^{1} \mathrm{H}$ STD NMR results of the cyclic dimer peptide interaction with mouse Muc1 $\mathrm{mAb}(6 \mathrm{~A} 4)$ compare to the linear monomer peptide GVTSAPD previously studied in this laboratory (see reference 13 for ${ }^{1} \mathrm{H}$ assignments and specific details of the linear peptide). ${ }^{12,13}$

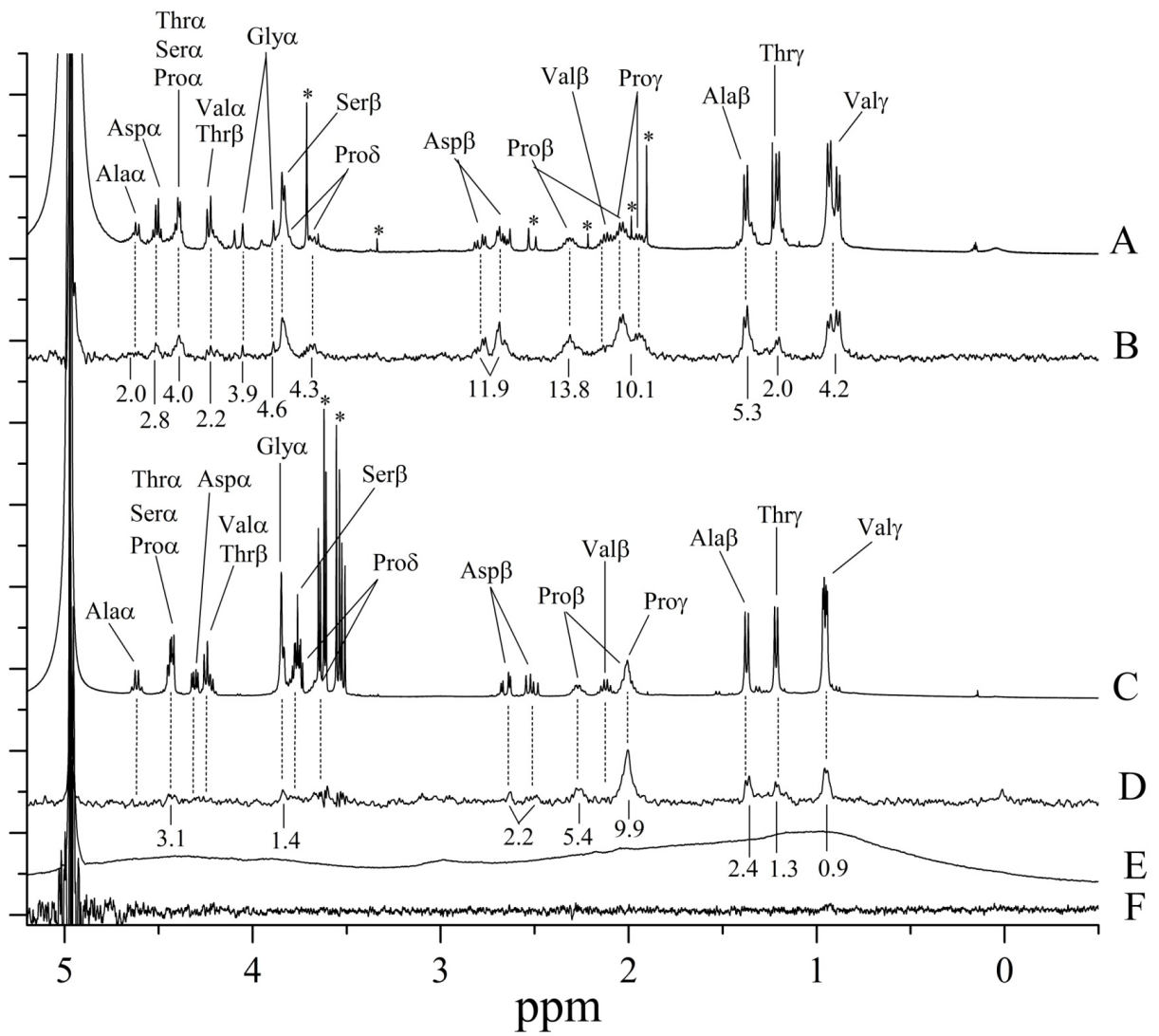

Figure 4. ${ }^{1} \mathrm{H}$ STD NMR spectra of peptides showing protons that give rise to STD peaks as they bind to mAb (6A4). Vertical dashed lines connect the STD peaks to their corresponding protons. A line broadening of $2 \mathrm{~Hz}$ was applied to the STD spectra. The numbers below the STD spectra are ASTD values for the corresponding STD peaks. The traces are: (A) ${ }^{1} \mathrm{H}$ NMR spectrum of a mixture of cyclic dimer peptide (cyclo-GVTSAPDGVTSAPD) and mAb; (B) STD NMR spectrum of cyclic dimer peptide plus mAb; (C) ${ }^{1} \mathrm{H}$ NMR spectrum of a mixture of linear GVTSAPD peptide plus $\mathrm{mAb}$; (D) STD NMR spectrum of linear GVTSAPD peptide plus $\mathrm{mAb}$ (spectra $\mathrm{C}$ and $\mathrm{D}$ are reprinted from reference 13 with the authors' permission); (E) Control STD spectrum of $\mathrm{mAb}$ in the absence of any peptide, only broad humps from the resonances of the $\mathrm{mAb}$ is observed; (F) Control STD spectrum of peptide in the absence of $\mathrm{mAb}$; there are no $\mathrm{mAb}$ peptide interactions, so no STD peaks observed. Peaks marked with “*” are contaminants.

There arose more STD NMR peaks for cyclic dimer peptide than for the monomer linear peptide. In the $\alpha \mathrm{H}-\beta \mathrm{H}$ regions, the results showed clear saturation transfer effects to all the protons of the Pro residue, although the ProHa was overlapped with the aH's of Ser and Thr. Other clear saturation transfer effects occurred at AspHa, $\mathrm{AspH} \beta, \operatorname{SerH} \beta, \mathrm{GlyH \alpha}$, and the methyl groups of Ala, Val and Thr, but the intensity of the Thr methyl group was weaker than those of Ala and Val. The unambiguous peaks corresponding to the protons with the tallest STD NMR signals were from $\mathrm{ValH} \gamma, \mathrm{AlaH} \beta, \mathrm{AspH} \beta, \mathrm{ProH} \beta$ and $\mathrm{ProH} \gamma$ (Figure 4). The Asp residue showed markedly enhanced STD peaks, which was not the case for the linear peptide. The intensities of the various STD peaks for all residues of the cyclic dimer peptide were higher than those of the linear peptide when comparing their amplification factor values. The total ASTD value of Pro and Asp residues were higher than those of other residues. 


\section{DISCUSSION}

The binding of specific monoclonal antibody to the linear sequence GVTSAPD was confirmed in a previous study. ${ }^{12,13}$ It is important to note that Pro6 in that peptide is crucial to the interaction between the peptide antigen and antibody. In this study, through STD NMR, we have determined that the cyclic dimer peptide with the sequence GVTSAPDGVTSAPD has antibody binding ability to Mucl mAb (6A4) with all its residues. Examination of the STD NMR spectrum showed that there are STD peaks corresponding to most of the resonances of the normal ${ }^{1} \mathrm{H}$ NMR spectrum, indicating that most protons of the residues on the cyclic dimer peptide are interacting with the antibody. The STD NMR peak intensity where the resonance of AlaHa is centered is essentially zero. At the methyl group region, the STD NMR peak intensity of ThrH $\gamma$ is weaker than those of Ala and Val. If there are no interactions occurring between the peptide and the antibody the STD spectrum would be a flat line without any peaks, and the higher the STD NMR peak intensity for a particular proton, the closer it is to the antibody. ${ }^{21}$ Inspection of the two STD NMR spectra showed that the STD peaks arose from the cyclic dimer peptide are more pronounced than those from the linear monomer peptide, which suggests the protons of the cyclic dimer peptide are closer to the antibody at the binding interface. The fact that almost all of the protons of all amino acid residues experienced STD effects or are interacting with the antibody suggests that this peptide intercalates its whole self into the binding pocket of the antibody compare to the linear version of the peptide (GVTSAPD), where mainly the Pro residue and the methyl groups have interactions. ${ }^{12,13}$ This may mean that the binding of the cyclic dimer peptide to the antibody is more effective (the dipeptide increases the local concentration of the ligand at the $\mathrm{mAb}$ binding site, which can also lead to an increase in the signal; not necessarily due to increased affinity). This can be expected because the cyclic dimer peptide has the same sequence on both sides of its molecule in reverse order. Base on the higher STD peak intensities of the Pro side chain protons, it appeared that the Pro residue is situated closer to the antibody at the binding pocket than any other residues similar to previous finding for the linear peptide.

It has been previously confirmed from substituted peptides studies that proline is a crucial residue in antibody recognition for this particular sequence. ${ }^{12,13}$ On the cyclic dimer peptide, the $\mathrm{H} \beta$ and $\mathrm{H} \gamma$ on proline displayed highest STD peaks suggesting that they are closer to the antibody binding interface or more accessible to it than the $\alpha \mathrm{H}$ and $\mathrm{\delta H}$ (Figure 4, spectrum $\mathrm{B}$ ). A conclusion can be drawn from the STD peaks observed for Pro, Val, Ala and Thr that the binding of this peptide to $\mathrm{mAb}$ (6A4) favors a hydrophobic peptide-antibody interface. The side chains of Val and Ala are hydrophobic and contain no heteroatoms, which may explain the similar methyl STD peak intensities (or ASTD values) between the two residues. On the other hand Thr, which contains a hydroxyl group on its $\beta$-carbon, has a side chain of lower hydrophobicity and displayed a lower STD peak intensity. This is consistent with the hypothesis that the antibody molecule prefers to interact with hydrophobic residues on the peptide chain. One striking contrast to this observation is the Asp residue. The side chain of Asp has a low degree of hydrophobicity yet it displayed a much more intense STD peak at the $\mathrm{H} \beta$ compared to the Thr $\mathrm{CH}_{3}$-group. The Asp side chain wouldn't be supported in a hydrophobic binding site, and therefore is expected to be oriented outside the suggested hydrophobic site. A possible explanation for this is the position of Asp preceded by Pro on the peptide chain, where its sequential location determines its binding property; additionally, a type of secondary structure formed on the cyclic peptide at this region that favors interaction with the antibody is not ruled out. Pro is an important residue in the binding interaction as all of its side chain protons displayed STD peaks. Because of this, Pro would be the residue that is physically nearest in contact with the antibody, and the residues flanking Pro in a cyclic peptide, such as Asp, could be fixed into a constraint that is preferred to interact with the antibody. A more dynamically limited Asp may favor the formation of intermolecular interactions with the antibody. A substitution of Asp for Ala may confirm the above suggested hydrophobic peptide-antibody interface. For the Gly residue, the two geminal protons are not equivalent; both experienced small saturation transfer effect and of about equal STD peak intensity, which suggests Gly is farther away from the binding 
interface. The result is inconclusive whether Ser is involved in the binding, as its $\mathrm{Ha}{ }^{1} \mathrm{H}$ NMR peak overlaps with the Ha's of Thr and Pro, and its $\mathrm{H} \beta$ has overlapping with one of the ProH $\delta$ peaks, contributing further ambiguity to that resonance (at $3.8 \mathrm{ppm}$ ). Because the Pro residue is known to be a binding residue, it is likely, at least, that part of the intensity of the peak appearing at $3.8 \mathrm{ppm}$ also belongs to ProH $\delta$. Additional studies will have to be pursued to determine exactly whether Ser is comprised in the peptide-antibody interface, however, it cannot be excluded because of the peak overlapping described. The fact that the cyclic dimer peptide has more interactions (binds better, not necessarily stronger) with the $\mathrm{mAb}$ compared to the monomer linear peptide is due to two reasons: 1). its residues are constrained in the cyclic peptide, and 2). the doubly repeat sequence in which either side of the peptide is identical in opposite direction allows it to have a higher probability of binding, a kind of double-edge sword property in term of binding to the antibody.

\section{CONCLUSION}

In conclusion, we have synthesized a cyclic dimer mucin peptide with the sequence GVTSAPDGVTSAPD and showed that it possesses Mucl monoclonal antibody binding activity by STD NMR. Almost all of the aliphatic protons of all its amino acid residues gave rise to STD NMR peaks suggesting that most of, if not all, its residues have interactions with the antibody because of its two identical chains of the peptide. Ambiguity does exist in the binding of Ser residue and some of the $\alpha H$ 's due to overlapping resonances. The results clearly showed that the protons of Pro residue and those of the methyl groups of Ala, Thr and Val have interactions with the antibody, signifying the importance of hydrophobic interaction. The data of this study may further suggest the potential usefulness of the cyclic dimer mucin peptide as an additional antigenic valence in immunotherapy of cancer research.

\section{ACKNOWLEDGEMENTS}

This research was supported by Faculty/Student Collaborative grants and Minority Mentoring grants from the University of Wisconsin-Eau Claire (UWEC) Office of Research and Sponsored Program. We thank the UWEC Chemistry Department for its support of many hours of NMR and LC-MS time, and supplies of chemicals.

\section{REFERENCES}

[1] Grinstead, J. S., Koganty, R. R., Krantz, M. J., Longenecker, M. B., and Campbell, P. A. (2002) Effect of Glycosylation on MUC1 Humoral Immune Recognition: NMR Studies of MUC1 Glycopeptide-Antibody Interactions, Biochemistry 41, 9946-9961. doi: 10.1021/bi012176z.

[2] Singh, R., and Bandyopadhyay, D. (2007) MUC1: A Target Molecule for Cancer Therapy, Cancer. Biol. Ther. 6, 481-486. doi: 10.4161/cbt.6.4.4201.

[3] Hollingsworth, M. A., and Swanson, B. J. (2004) Mucins in Cancer: Protection and Control of the Cell Surface, Nature 4, 45-60. doi: 10.1038/nrc1251.

[4] Möller, H., Serttas, N., Paulsen, H., Burchell, J. M., Taylor-Papadimitriou, J., and Meyer, B. (2002) NMR-based determination of the binding epitope and conformational analysis of MUC-1 glycopeptides and peptides bound to the breast cancer-selective monoclonal antibody SM3, Eur. J. Biochem. 269, 1444-1455. doi: 10.1046/j.1432-1033.2002.02787.x.

[5] Kuemmel, A., Single, K., Bittinger, F., Faldum, A., Schmidt, L. H., Sebastian, M., Micke, P., Taube, C., Buhl, R., and Wiewrodt, R. (2009) TA-MUC1 epitope in non-small cell lung cancer, Lung Cancer 63(1), 98-105. doi: 10.1016/j.lungcan.2008.04.005

[6] Gendler, S., Taylor-Papadimitriou, J., Duhig, T., Rothbard, J., and Burchell, J. (1988) A highly immunogenic region of a human polymorphic epithelial mucin expressed by carcinomas is made up of tandem repeats, J. Biol. Chem. 263: 12820-12823. 
[7] Tarp, M. A., Sørensen, A. L., Mandel, U., Paulsen, H., Burchell, J., Taylor-Papadimitriou, J., and Clausen, H. (2007) Identification of a novel cancer-specific immunodominant glycopeptide epitope in the MUC1 tandem repeat, Glycobiology 17(2), 197-209. doi:10.1093/glycob/cwl061.

[8] Vassilaros, S., Tsibanis, A., Tsikkinis, A., Pietersz, G. A., McKenzie, I. F., and Apostolopoulos, V. (2013) Up to 15-year clinical follow-up of a pilot Phase III immunotherapy study in stage II breast cancer patients using oxidized mannan-MUC1, Immunotherapy 5(11), 1177-1182. doi: 10.2217/imt.13.126.

[9] Deguchi, T., Tanemura, M., Miyoshi, E., Nagano, H., Machida, T., Ohmura, Y., Kobayashi, S., Marubashi, S., Eguchi, H., Takeda, Y., Ito, T., Mori, M., Doki, Y., and Sawa, Y. (2010) Increased Immunogenicity of Tumor-Associated Antigen, Mucin 1, Engineered to Express $\alpha$-Gal Epitopes: A Novel Approach to Immunotherapy in Pancreatic Cancer, Cancer Res. 70(13), 5259-5269. doi: 10.1158/0008-5472.CAN09-4313.

[10] Kotera, Y., Fontenot, J. D., Pecher, G., Metzgar, R. S., and Finn, O. J. (1994) Humoral Immunity against a Tandem Repeat Epitope of Human Mucin MUC-1 in Sera from Breast, Pancreatic and Colon Cancer Patients, Cancer Res. 54, 2856-2860.

[11] Kovjazin R., Horn G., Smorodinsky N. I., Shapira M. Y., and Carmon, L. (2014) Cell Surface-Associated Anti-MUC1-Derived Signal Peptide Antibodies: Implications for Cancer Diagnostics and Therapy, PLoS ONE 9(1): e85400. doi: 10.1371/journal.pone.0085400.

[12] Her, C., and Yang, T., (2012) Antibody Binding Study of Mucin Peptide Epitopes. Division of Biological Chemistry, 243rd ACS meeting, San Diego, CA., March 25-29.

[13] Her C., Westler W. M., and Yang T. (2013) Significance of Proline Residue on Short Mucin Peptide Interactions with Mouse MUC1 Monoclonal Antibody Studied by Saturation Transfer Difference NMR Spectroscopy, JSM Chem 1(1): 1004.

[14] Chan, W. C., and White, P. D., (ed.), (2000) Basic Procedures, in Fmoc Solid Phase Peptide Synthesis, A Practical Approach, Oxford University Press, UK, pp. 41-74.

[15] Demmer, O., Frank, A. O., and Kessler, H. (2009) Design of Cyclic Peptides, in Peptide and Protein Design for Biopharmaceutical Applications (ed., K. J. Jensen), John Wiley \& Sons, Ltd, Chichester, UK, pp. 133-166.

[16] Alcaro, M. C., Sabatino, G., Uziel, J., Chelli, M., Ginanneschi, M., Rovero, P., and Papini, A. M. (2004) On-resin Head-to-tail Cyclization of Cyclotetrapeptides: Optimization of Crucial Parameters, J. Peptide Sci. 10, 218-228. doi: 10.1002/psc.512.

[17] Chan, W. C., and White, P. D., (ed.), (2000) RP-HPLC using lipophilic chromatography probes, in Fmoc Solid Phase Peptide Synthesis, A Practical Approach, Oxford University Press, UK, pp. 269-276.

[18] Berger, S., and Braun, S., (ed.), (2004) in 200 and More NMR Experiments, A Practical Course, WILEY-VCH Verlag GmbH \& Co. KGaA, Weinheim, Germany, pp. 422-425.

[19] Piotto, M.., Saudek, V., and SKlenář, V., (1992) Gradient-tailored excitation for single-quantum NMR spectroscopy of aqueous solutions, J. Biomol. NMR 2(6), 661-665. doi: 10.1007/BF02192855.

[20] Mayer, M., and Meyer, B. (2001) Group Epitope Mapping by Saturation Transfer Difference NMR To Identify Segment of a Ligand in Direct Contact with a Protein Receptor, J. Am. Chem. Soc. 123, 6108-6117. doi: 10.1021/ja0100120.

[21] Viegas A., Manso J., Nobrega F. L., and Cabrita E. J. (2011) Saturation-Transfer Difference (STD) NMR: A Simple and Fast Method for Ligand Screening and Characterization of Protein Binding, J. Chem Educ. 88, 990-994. doi: 10.1021/ed101169t.

[22] Sewald, N., and Jakubke, H.-D. (ed.), (2002) Synthesis of Special Peptides and Peptide Conjugates in Peptides: Chemistry and Biology, WILEY-VCH, Verlag GmbH, Weinheim, Germany, pp. 311-337. 


\section{ABOUT THE STUDENT AUTHOR}

Cheng Her graduated with a B.S. degree in Biochemistry/Molecular Biology in 2012 from the University of Wisconsin-Eau Claire. He was a McNair program scholar and had conducted undergraduate research for two years in the Chemistry Dept. He is now attending graduate school in biophysical chemistry at the University of Minnesota-Twin cities, MN.

\section{PRESS SUMMARY}

In this project we intended to synthesize a monomeric cyclic mucin peptide, instead cyclodimerization led to a byproduct that is isolatable from impurities and worth examining for its ability to bind MUC1 mucin monoclonal antibody. The cyclic dimer peptide may be useful to serve as an antigenic epitope for induction of immune response against the mucin antigen.

\section{$\mathrm{AJUR}$ Volume 12 | Issue 4 | November 2015}




\title{
Methods for Essential Tremor Assessment: Acoustic Tremor Monitoring (ATM) and Rhythmic Spirals (RS) Methods
}

\author{
Emily Hart, Caroline Chow, Patricia Stan \& Daniel King \\ Department of Chemistry, Taylor University, Upland, IN \\ Students: emily_hart1@taylor.edu,cchow21490@gmail.com \\ Mentors: ptstan@taylor.edu,dnking@tayloru.edu
}

\begin{abstract}
There are several techniques of monitoring essential tremors, but there is not yet a standard method developed for the field. A quantitative way to track effects of medication and/or lifestyle treatment would be beneficial for future research in prevention or regression of essential tremors. The two methods evaluated are acoustic tremor monitoring (ATM) and rhythmic spirals (RS). The novel ATM measurement quantifies frequency and amplitude quickly and cost effectively. The tremor patient holds a microphone close to a speaker playing a single frequency tone. The Doppler Effect caused by the shaking microphone distorts the sound recording, and the encoded tremor information can be retrieved by using the Fast-Fourier Transform algorithm. The second method, RS, can be used by patients at home to measure frequency. The RS method is similar to the classic Archimedes spirals, but uses a different form and is timed which allows for the calculation of tremor frequency. The RS and ATM methods produce statistically similar frequency measurements, although ATM has greater precision.
\end{abstract}

\section{KEYWORDS}

Essential Tremors, Archimedes Spiral, Accelerometry, Spiral Analysis, Acoustic Tremor Monitoring, Rhythmic Spirals.

\section{INTRODUCTION}

Essential tremor (ET) is a condition that affects millions of Americans and for which there does not currently exist a standard quantitative clinical assessment method. The progression of the disorder and its correlation to other neurodegenerative disorders are poorly understood and, consequently, patient care and research could be dramatically improved with the development of a simple, effective quantitative assessment method. Tremors are defined as repetitive, rhythmic, involuntary, oscillations of a body part, and are the most common of all movement disorders., ${ }^{1,2,3}$ Two primary approaches have been used for classifying tremors. One system focuses on when the tremors occur. If a tremor is produced when the affected part is relaxed it is called a static tremor. ${ }^{1}$ If a tremor starts during a voluntary muscular contraction it is called an action tremor. Action tremors can be further broken down into more specific classifications including postural, isometric, kinetic, task-specific, and intentional tremors. ${ }^{1}$ The second system of classification focuses on the underlying cause of the tremor, either physiological or pathological., ${ }^{1,2}$ Physiological tremors happen in all humans and can be seen or unseen and often become more visible within specific situations, like being tired or hungry, and with age., ${ }^{1,2}$ Pathological tremors are associated with neurological disorders and include cerebellar, psychogenic, orthostatic, neuropathic, parkinsonian, and essential tremors. ${ }^{2}$ Despite the fact that many ET cases do not seek medical help, ${ }^{4}$ there is still an estimated 10 million Americans diagnosed with essential tremors which makes it the most common pathological tremor. ${ }^{5}$

Recent studies about the progression of ET indicate subjects experience a 3-5\% increase in the 
Total Tremor Scale (total number of tremor occurrences) or a 12\% increase in the Tremor Rating Scale (tremor severity) each year. ${ }^{6,7}$ Both scales are based on the visible observation of tremor symptoms. Although both studies utilized expert movement disorder neurologists to make the observations, inconsistencies were found between the calculated disease progression rates of the two methods. Additionally, studies have found ET patients to be at increased risk for other diseases or disabilities including cognitive problems (speech and memory), anxiety, depression, social phobia, balance, hearing issues, Parkinson's disease and dementia. ${ }^{4}$ As a result of these new findings, long term studies of ET patients are needed, utilizing a standard methodology to obtain consistent and quantitative measurements of the progression or regression of the disease.

Many techniques have been developed to measure the frequency and amplitude of tremors. Many physicians employ different qualitative severity tests, including questionnaires, that use numbered scales to rank the severity or intensity of tremors. ${ }^{1,2}$ Drawings like the Archimedes spiral are also being used to watch the progression of the tremor ${ }^{3,8}$ and to compare with other previously diagnosed patients. ${ }^{2}$ More sophisticated quantitative ways of measuring frequency and amplitude electronically are being created; a few methods include accelerometry, electromyography (EMG), magnetic tracker system, active optical markers, gyroscopes, spirography, and spiral analysis. ${ }^{1,2,3,9}$ However, despite the variety of qualitative methods and newer quantitative methods, physicians have yet to embrace a standard assessment method, perhaps because the qualitative tests are too subjective and the quantitative methods are too inaccessibly high tech to be clinically useful.

In an attempt to develop two complimentary methods, one that could easily be used by patients at home and one more robust method for clinicians, to measure tremor frequency and monitor tremor progression over time, we report here the novel Acoustic Tremor Monitoring and Rhythmic Spiral methods. The Acoustic Tremor Monitoring (ATM) method is a fast, easy, non-invasive method to measure frequency and observe the amplitude of a tremor. ATM uses sound waves that are distorted by a tremor and visualized with the Fast Fourier Transform algorithm. The Rhythmic Spiral (RS) method is an adaptation of the classic Archimedes spiral exercise already in use, performed at a specific rate so as to reveal tremor frequency information, and would be simple for patients to perform at home to estimate their tremor frequency.

\section{METHODS}

IRB approval was granted for this work. The pilot study participant was provided an Informed Consent, and both written and oral description of the project. The subject was asked to hold a microphone several times with each hand to collect the ATM signal and to complete the RS writing assessment several times to collect the RS data. The total involvement of the subject required less than two hours.

\section{Acoustic Tremor Monitoring: Clinical assessment}

The Acoustic Tremor Monitoring method can be used to analyze tremor frequency and amplitude from the Doppler distortion imparted into a sound recording when the microphone is held by a tremor subject (Figure 1). An inexpensive desktop microphone is used to record a sound file, held at approximately 6 inches from the $2000 \mathrm{~Hz}$ tone source. The Fast Fourier Transform (FFT) algorithm, a standard function of data processing software package Origin9.0 (OriginLab) is used to convert the sound wave to a frequency spectrum with respect to the magnitude of the signal. Initially, the microphone was exposed to a variety of conditions of mechanical oscillations that mimic hand tremors. These samples were recorded in WMA files and then converted into WAV files through a free online converter ${ }^{10}$ so that they could be processed by the Origin 9.0 software program. Frequency spectra are normalized to the most intense frequency $(2000 \mathrm{~Hz})$ and displayed as 100 percent relative intensity. 


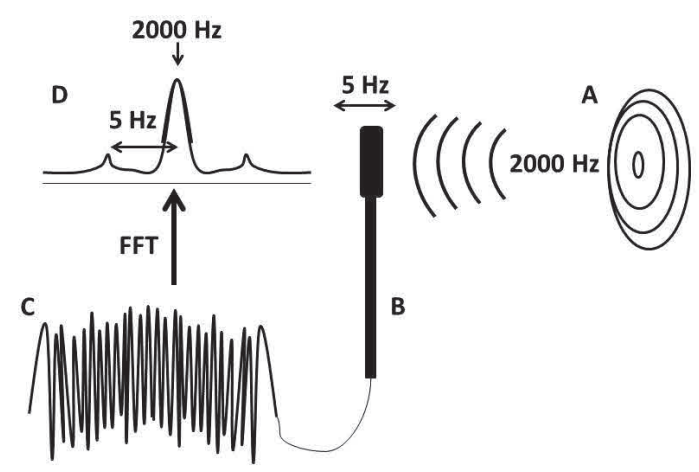

Figure 1. A schematic representation of the experimental setup is shown. (A) A $2000 \mathrm{~Hz}$ tone is played and recorded by a standard computer microphone (B) while being held by a subject. The resulting time-domain sound file (C) is processed by Fourier transform to yield frequency-domain spectrum (D). The tremor frequency and source tone frequency information is revealed in the frequency spectrum.

\section{Effect of Tremor Frequency on ATM Signal}

To evaluate the effect of tremor frequency on the ATM signal, a Gyrotory Water Bath Shaker, Model G76, was used with a microphone taped to one of the shaking bars. The shaker has a variable speed dial that allowed for varying speed and, consequently, frequency. A high pitch tone at 2000 $\mathrm{Hz}$ was played approximately 6 inches from the microphone, and recorded for about 5 seconds at each different speed setting, 1 to 5 .

\section{Effect of Tremor Amplitude on ATM Signal}

To evaluate the effect of tremor amplitude, or distance the microphone travels, on the ATM signal, the microphone was oscillated for varying distances but at a fixed frequency. Paths of half an inch, one inch, two inches, and three inches were marked on a piece of paper. A digital metronome was played at 208 beats per minute into a pair of headphones. With the high pitch tone $(2000 \mathrm{~Hz})$ being played approximately 6 inches from the microphone and while listening to the metronome through headphones, the microphone was manually oscillated the designated distances to the constant beat of the metronome. The signal was recorded for about 5 seconds.

\section{Rhythmic Spiral: Home assessment}

The Rhythmic Spiral method attempts to control the speed at which the traditional hand written Archimedes spiral is performed, providing a known and consistent rate, in order to impart meaningful tremor frequency information into the produced drawing. The spiral pattern used consists of 6 loops drawn side by side to make the shape of a spring (Figure 2). With a metronome playing at 40 beats per minute, the subject is asked to trace the spring while trying to hit the bottom of each loop on the beat. When finished the entire pattern should be completed in 9 seconds. The imperfections (zigzags) visible in the trace are the result of the tremor, and each tremor event results in one zigzag motion. Therefore, each zigzag (right - left pair) is counted as a single event. Then, the number of observed events is divided by 9 seconds to produce a solid estimate for the frequency of the tremor. The pattern can be repeated several times to calculate an average tremor frequency.

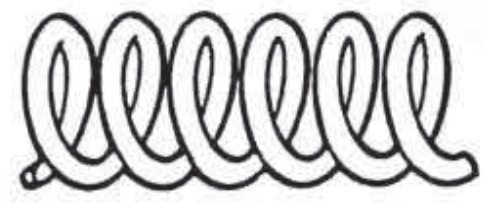

Figure 2. A sample spiral template pattern is shown. 


\section{RESULTS AND DISCUSSION:}

ATM

Acoustic Tremor Monitoring (ATM) takes advantage of the Doppler distortion that occurs when a monochromatic tone is recorded by a microphone that is shaking. Just like the higher and lower pitches heard when a police car and siren pass by quickly, the ATM picks up the played tone at its original frequency and at the original frequency plus and minus the frequency of the shaking. In an initial attempt to assess if the ATM method is sensitive enough to observe the shaking hand of an ET subject, a volunteer who presents with moderate ET in the right arm (dominant) but symptom free in the left arm (non-dominant) was selected. ET often develops first in the dominant hand, as it did for this subject. A 5 second recording of the monochromatic $2000 \mathrm{~Hz}$ tone with the microphone sitting on the table was collected to serve as a blank. A 5 second negative control was collected with the subject holding the microphone in the symptom free left hand. Then, a similar recording with the subject's ET affected right was collected. The frequency spectra are shown in Figure 3. As expected, two symmetrical side bands appeared in the tremor signal, likely resulting from the Doppler shift in the recorded frequencies. The correlation of these side bands to the frequency and amplitude of the tremor is evaluated below. Encouragingly, the negative control strongly resembles the blank and is easily distinguishable from the ET affected hand.

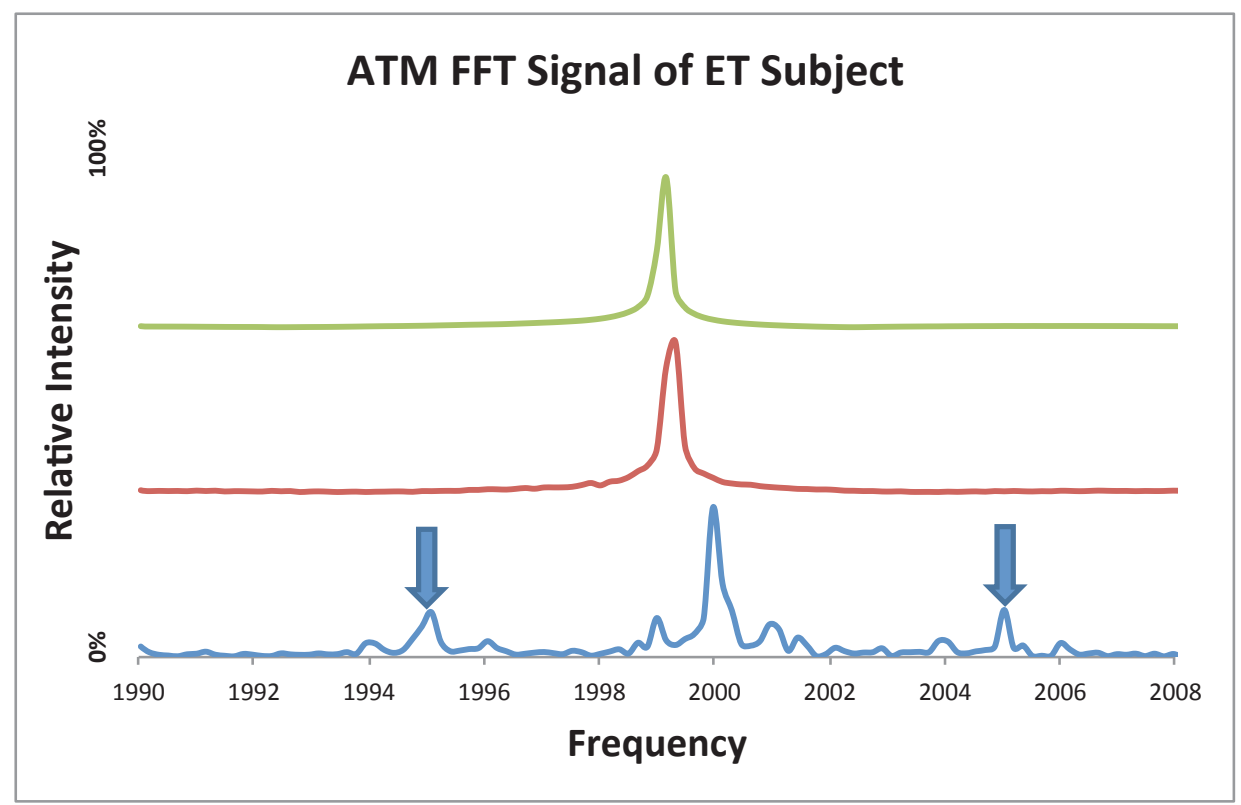

Figure 3. ATM signals for the blank $2000 \mathrm{~Hz}$ signal (green), the negative control with the subject's asymptomatic left hand (red), and the sample with the subject's symptomatic right hand (blue) are shown. The arrows point to the Doppler shifted side bands that are indicative of a shaking microphone.

\section{Effect of Tremor Frequency on ATM Signal}

To assess the effect of tremor frequency, the microphone was attached to a water bath shaker, and $2000 \mathrm{~Hz}$ recordings were made at five different speed settings. As the speed setting was increased, and consequently frequency, the Doppler peaks grew farther apart from one another. Predicting that the distance between the principle peak and each Doppler peak is the frequency at which the microphone oscillates, ten oscillations were observed and timed to determine the actual shaker frequency. Averaging four trials at each speed, the frequency of the shaker speeds 1-5 were determined to be $0.41,1.12,1.82,2.59$, and $3.37 \mathrm{~Hz}$, respectively. The frequencies determined by the ATM splitting distances were very similar to the manually determined frequencies of the shaker 
(NA, 1.08, 1.75, 2.58, and $3.39 \mathrm{~Hz}$ ). The similarity is statistically significant $(\mathrm{p}<0.05)$, and when plotted against each other correlate with an $\mathrm{R}^{2}$ value of 0.9995 . The ATM signals for speeds $2-5$ are displayed in Figure 4. It is noteworthy that the Doppler shifted peaks were not resolved for the slowest frequency $(0.41 \mathrm{~Hz})$. The results suggest an apparent lower frequency limit for the ATM method; however, it is far slower than the typical ET frequencies, 3-12 Hz. The slight shifting of the spectra along the frequency axis between trials appears to be a result of the slight inconsistency of the source tone. The fact that the side bands shift proportionally with the source peak, $2000 \mathrm{~Hz}$, suggests that the measured tremor frequency, the distance between the source peak and the side bands, will be unaffected.

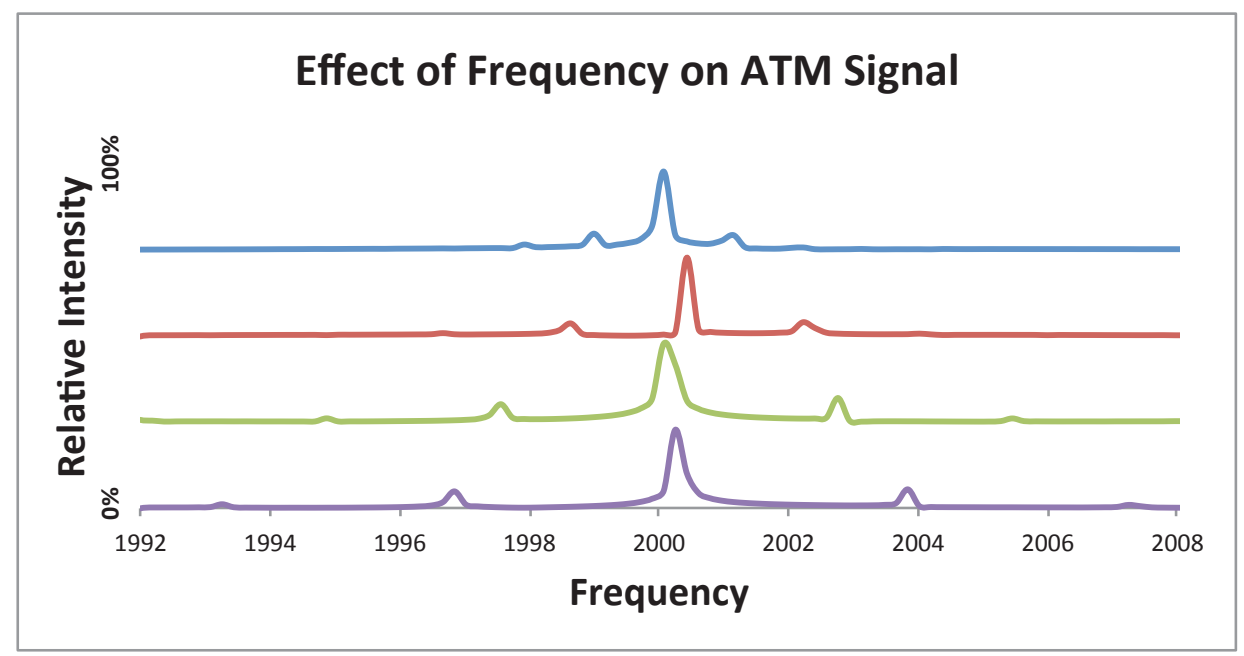

Figure 4. Individual, normalized ATM signals at various mechanical shaker speed settings are shown: speed $2=1.08 \mathrm{~Hz}$ (blue), speed $3=1.75 \mathrm{~Hz}$ (red), speed $4=2.58 \mathrm{~Hz}$ (green), and speed $5=3.39 \mathrm{~Hz}$ (purple).

\section{Effect of Tremor Amplitude on ATM Signal}

To assess the effect of tremor amplitude on the ATM signal, a set of tests were performed where the distance the microphone traveled was varied while holding the oscillation frequency constant. Using a metronome with headphones to maintain a constant frequency, the microphone was oscillated through a set of various distances marked on a table to simulate a variety of tremor amplitudes at 208 beats per minute. Manually translating the microphone through one oscillation (back and forth through the measured distances) for each beat produced a frequency of $3.47 \mathrm{~Hz}$. The ATM data collected for these trials produced an average measured frequency of $3.53 \mathrm{~Hz}(1.7 \%$ difference). The Doppler spacing remained constant for these trials indicating that a consistent oscillation frequency was maintained. The Doppler shifted peaks' heights, however, varied significantly with amplitude. As the amplitude of the oscillations increased, the height of the Doppler shifted peaks increased systematically, although not linearly (linear R2 $=0.961$, exponential R2 $=0.9986$ ). The Doppler shifted peak heights for the three distances (1, 2, and 3 inches) were $4.9 \%, 12.2 \%$, and $27.5 \%$ of the principle $2000 \mathrm{~Hz}$ peak, respectively (Figure 5). Although, this trend was expected to be linear, the deviation from linearity may be a result of slight inconsistencies in manually translating the microphone specified distances. 


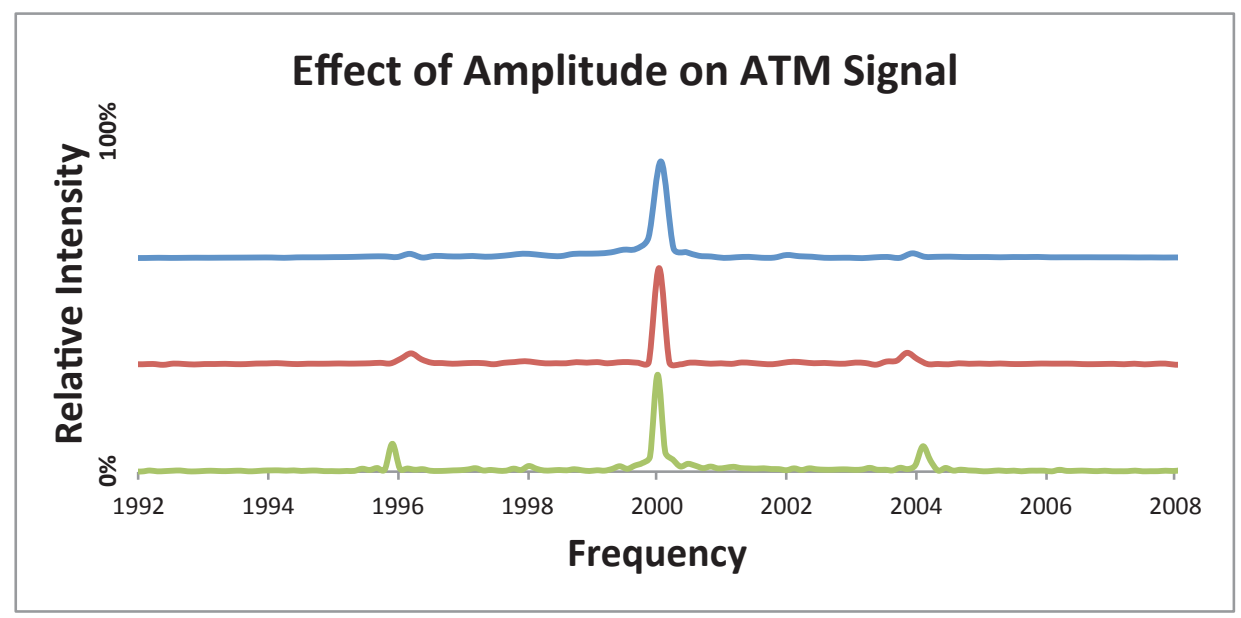

Figure 5. Normalized ATM signals for $3.47 \mathrm{~Hz}$ oscillations over distances of 1 inch (blue), 2 inches (red), and 3 inches (green) are shown.

\section{Rhythmic Spiral Home Assessment}

In addition to the clinical ATM method, the classic Archimedes spiral was adapted to provide tremor patients with a cheap and simple way to assess their tremor frequency at home. Once a patient becomes comfortable tracing the spiral pattern to a beat and counting the tremor features, the frequency of an essential tremor can be quickly measured. The same ET volunteer who participated in testing the ATM methods, with moderate essential tremor in the right hand, also performed the RS test. Although the RS test could in principle be performed on a non-dominant hand, most subjects will likely lack the dexterity to do so. Fortuitously for this analysis, ET is often more significantly developed and most inconvenient in the dominant hand. Figure 6 shows a sample RS test with each zigzag pair marked as a single event. The total number of tremor events are counted and divided by 9 to calculate the tremor frequency estimate. It is reasonable to expect that the amplitude may correlate to the size of the zigzags, but we have yet to devise a method for evaluating this hypothesis. The RS test was performed 19 times on the subject to yield an average observed tremor frequency of $4.7 \mathrm{~Hz}$ (standard deviation $= \pm 0.43 \mathrm{~Hz}$ ). The average ATM frequency measurements for the same subject were $5.0 \mathrm{~Hz}$ (standard deviation $= \pm 0.11 \mathrm{~Hz}$ ). Both the RS and the ATM method are compared in Figure 7 below. The resulting frequencies from both methods agree quite well, with the ATM method showing greater precision. Both methods were sensitive enough to evaluate this moderate essential tremor subject.

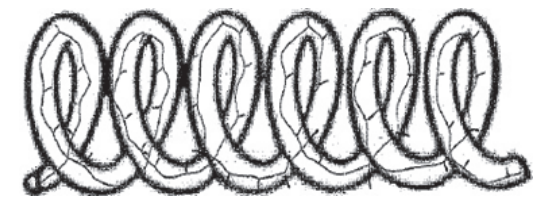

Figure 6. A sample RS sample from an ET subject is shown. The RS trace shows the typical zigzag pattern, where each zigzag pair is indicative of a single tremor event. The total events divided by the total time, 9 sec., yields the tremor frequency. 


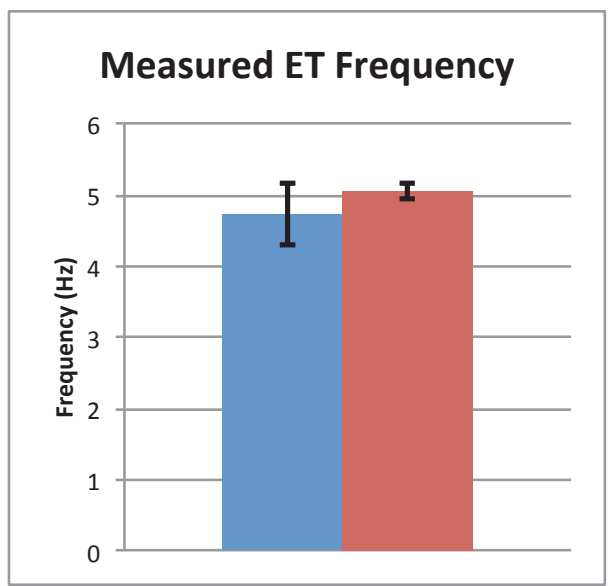

Figure 7. The average measured frequency from 19 RS tests, $4.7 \mathrm{~Hz}$, and standard deviation, $\pm 0.43 \mathrm{~Hz}$, for an ET subject is shown in blue. The average measured frequency from 4 ATM tests, $5.0 \mathrm{~Hz}$, and standard deviation, $\pm 0.11 \mathrm{~Hz}$, for the same ET subject is shown in red.

\section{CONCLUSIONS AND FUTURE WORK}

The ATM and RS methods are sufficiently accurate, precise, and accessible to warrant further evaluation as to their usefulness for the routine monitoring of essential tremors and potentially for diagnosis. ATM may be capable of accurately measuring tremor frequency and observing the change in amplitude of a tremor with minimal technological requirements. Although ATM has been cast here as a potential clinical method, it is reasonable to imagine patients collecting the ATM recordings on their own at home and electronically sending the file to a clinician for evaluation. The ATM method has the distinct advantage over RS in that its frequency measurements are more precise and it can quantitatively evaluate amplitude. The RS method is a completely low tech variation of what many ET patients are already doing, and can be used to easily keep track of the frequency at home. The RS method provides reliable frequency measurements and may prove more accessible to the clinical community because of its similarity to the Archimedes spirals currently being used. Although only tested with one subject, the tremor frequencies measured by ATM and RS are consistent and complementary warranting their further evaluation in a larger ET population.

Future collaborations with physicians specializing in ET to study a larger ET population will be necessary to evaluate the full potential of the ATM and RS methods. A large ET population should be evaluated by ATM and the results compared to the subjective scale assessments performed by the clinicians. Finding tangible correlations between ATM frequency and amplitude values and physician assessment scales would not only substantiate the ATM method but could also provide valuable insight into the progression of ET if patients are monitored over a long period of time. Additionally, the same ET patients would perform RS tests on their own at home to allow for not only a large scale comparison of RS and ATM results, but also the evaluation of the precision and accuracy patients are able to achieve performing the test at home.

For a given ET patient, tremors may fluctuate significantly for reasons such as fatigue, hunger, and caffeine ingestion. Consequently, having annual qualitative clinical assessments may not only be quite subjective, but will be susceptible to the temporal variations of the tremor itself. Simply put, patients can have good days and bad days. Similarly, because of these same natural variations it is difficult for patients to assess the effectiveness of a newly prescribed treatment, like alcohol or beta-blockers, since so many things can affect the tremor symptoms. A quantitative assessment tool that could be used routinely in order to yield reliable and meaningful average tremor measures could dramatically improve the specificity of the care patients could receive, greatly increase the chances for researchers to find meaningful correlations between ET progression and the development of other neurological and psychological conditions, and empower ET patients who generally feel helpless and hopeless with regard to their ET progression. 


\section{REFERENCES}

[1] Mansur, P.H.G., Cury, L.K.P., Andrade, A.O., Pereira, A.A., Miotto, G.A.A., Soares, A.B., Naves, E.L.M. (2007) A Review on Techniques for Tremor Recording and Quantification Biomedical Engineering 35, 343-362.

[2] Andrade, A. O., Pereira, A. A., Soares, M. F., de Almeida, G. L. C., Paixão, A. P. S., Fenelon, S. B., \& Dionisio, V. C. (2012). Human Tremor: Origins, Detection and Quantification, in Practical Applications in Biomedical Engineering (Andrade, A. O., Pereira, A. A., Naves, E. L. M., Soares, A. B., Ed.) pp 3-24, InTech.

[3] Almeida, M. F. S., Cavalheiro, G. L., Pereira, A. A., \& Andrade, A. O. (2010). Investigation of age-related changes in physiological kinetic tremor. Annals of biomedical engineering, 38 (11), 34233439.

[4] Louis, E. D., \& Okun, M. S. (2011). It is time to remove the 'benign' from the essential tremor label. Parkinsonism \& related disorders, 17 (7), 516-520.

[5] Facts about Essential Tremor, International Essential Tremor Foundation, http://www.essentialtremor.org/wp-content/uploads/2013/07/FactSheet012013.pdf (accessed Oct 2014)

[6] Putzke, J. D., Whaley, N. R., Baba, Y., Wszolek, Z. K., \& Uitti, R. J. (2006). Essential tremor: predictors of disease progression in a clinical cohort. Journal of Neurology, Neurosurgery \& Psychiatry, 77 (11), 1235-1237.

[7] Louis, E. D., Agnew, A., Gillman, A., Gerbin, M., \& Viner, A. S. (2011). Estimating annual rate of decline: prospective, longitudinal data on arm tremor severity in two groups of essential tremor cases. Journal of Neurology, Neurosurgery \& Psychiatry, 82 (7), 761-765.

[8] Ellias, S. A. (1993). Essential tremor. Rhode Island Medicine, 76, 563-566.

[9] Diagnostic Tests of Motor Function, CMPL, Columbia University Medical Center, Department of Neurology, http://cmpl.columbia.edu/diagnostic.html\#top (accessed Oct 2014).

[10] Burkard, J., Online Audio Converter, http://media.io/ (accessed Oct 2014)

\section{ABOUT THE STUDENT AUTHORS}

Emily Hart is a senior at Taylor University working towards a BA degree in Chemistry. Caroline Chow was a 2012 Taylor University graduate and is now a chemistry teacher at Jac-Cen-Del High School, Osgood, IN.

\section{PRESS SUMMARY}

We developed two methods to assess essential tremors: acoustic tremor monitoring (ATM) and rhythmic spirals (RS) methods. These complementary methods are easy, non-invasive techniques that are likely to measure essential tremors quickly and inexpensively. ATM uses sound waves to monitor the frequency and amplitude of the tremor. RS is an at home method adapted from Archimedes spirals which gives a quick estimate of a tremor's frequency. 
Print Edition ISSN 1536-4585

Online Edition ISSN 2375-8732 